


\title{
Arrhythmia termination using Global Optogenetic Stimulation in ChR2 mice hearts
}

\author{
Dissertation \\ for the award of the degree \\ "Doctor rerum naturalium" \\ of the Georg-August-Universität Göttingen
}

within the doctoral program Physics of Biological and Complex Systems of the Georg-August University School of Science (GAUSS)

submitted by

Raúl Alejandro Quiñonez Uribe from Ensenada, Mexico

Göttingen, 2020 


\section{Thesis Committee}

Prof. Dr. Stefan Luther, Research Group Biomedical Physics, Max Planck Institute for Dynamics and Self-Organization

Prof. Dr. André Fiala, Dept. of Molecular Neurobiology of Behaviour, University of Göttingen

Prof. Dr. Jörg Enderlein, III. Institute of Physics, University of Göttingen

\section{Members of the Examination Board}

Referee: Prof. Dr. Stefan Luther

$2^{\text {nd }}$ Referee: Prof. Dr. André Fiala

Further members of the Examination Board:

Prof. Dr. Jörg Enderlein

Dr. Andreas Neef, Campus Institute for Dynamics of Biological Networks, Max-Planck-Institute for Experimental Medicine

Prof. Dr. Luis A. Pardo, Dept. of Molecular Biology of Neuronal Signals Max Planck Institute for Experimental Medicine

Brett Carter, Ph.D., Synaptic Physiology and Plasticity, European Neuroscience Institute

Date of oral examination: August $27^{\text {th }}, 2020$ 


\section{Dedication}

Para mi mamá, mis hermanos y la familia. Porque el tiempo vivido fuera de Ensenada ha sido tiempo lejos de ustedes.

To my mom, my brothers and family. Since the time spent away from Ensenada has been time spent away from you. 


\section{Acknowledgments}

First of all, I would like to thank my supervisor Stefan Luther for giving me the opportunity to join the Biomedical Physics research group more than 4 years ago and for guiding me throughout my doctoral project and for enthusiastically and generously sharing his knowledge in pursue of my development as a researcher. I would also like to thank Claudia Richter for teaching me plenty of skills and subjects from the laboratory and from the field of cardiac optogenetics. Also, thanks a lot for the great help in writing the paper.

Special thanks to my Thesis Advisory Committee Jörg Enderlein and André Fiala for interestingly attending the meetings and thoughtfully evaluating my progress, providing me with their knowledge and giving me feedback when necessary. Also to my Examination Board Jörg Enderlein, André Fiala, Andreas Neef, Luis Pardo and Brett Carter for showing interest in my research accepting the invitation to my thesis defense, as well as for the interesting questions they will ask on this day.

I would like to thank my colleagues for making the experience of working at this research group very smooth, interesting and friendly. Thanks a lot for helping, for the discussions and for the different activities organized. From everyone I learned about science and about life and culture. Old and new colleagues I would like to thank: Jan Christoph, Tariq, Henrik, Sebastian B., Sebastian S., Svetlana, Laura, Thomas, Johannes, Filippo, Florian, Baltasar, Justine, Jan L. Special thanks to Vineesh for sharing the Be-Optical experience together and to Sayedeh for sharing many talks on cardiac optogenetics and doctorate life.

I would also like to hugely thank Marion and Tina. Without all your help this thesis would not have been possible. You helped me many times with different situations and definitely made working at the laboratory a lot easier. I would like to thank Annette, Claudia and Janna for the help provided in the editing of this thesis. Special thanks to Annette for the many comments that helped improve the quality of my writing.

Thanks to all the friends, old and new that were part of my life here in Göttingen and made the free time a lot nicer. And thanks to my family for always being there. 


\section{Abstract}

Cardiac arrhythmias represent a health threat worldwide that can end in sudden death. Treatments which include electrical shocks, anti-arrhythmic drugs and ablation show various disadvantages such as discomfort for the patients, side and pro-arrhythmic effects as well as lack of specificity. Moreover, arrhythmias display complex spatio-temporal behaviors making their study a challenging task. Cardiac optogenetics is an emerging field used to enable photo-control of cardiomyocytes by inscribing in them light-sensitive ion channels such as Channelrhodopsin-2 (ChR-2). Optogenetic stimulation offers a new dimension to investigate cardiac electrophysiology, from high spatial and temporal resolution to cell specificity and intensity dependent effects. Previous studies have shown the capability of arrhythmia control and termination using optogenetics in ChR-2 transgenic mice hearts. However, these studies relied on local stimulation, which requires either high intensities or long pulses. In this doctoral thesis I seek to investigate the advantages of globally illuminating the heart as a method of optogenetic cardioversion. In order to do so, I first characterized the attenuation of light by the cardiac tissue as well as the response to light stimulation of our model of ChR-2 transgenic mice hearts using the Langendorff-perfusion technique. The effect of the intensity, pulse width and diameter of the fiber under different experimental conditions involving tyrode, blebbistatin and the voltage sensitive dye Di-4-ANBDQPQ were investigated in this first step. Next, I designed an experimental setup that allowed global illumination of the isolated heart. Arrhythmia induction was facilitated using the drug pinacidil before stimulating the hearts using pulses of varying intensities and lengths. I also analyzed the efficiency of optogenetic cardioversion to different arrhythmia wave morphologies, and lastly investigated the time it takes an arrhythmia to be terminated using light in order to better understand the mechanisms behind this phenomenon. My results show that both intensity as well as the length of the pulse affect every aspect of optogenetic stimulation. With a higher effect from intensity, increasing these parameters leads to a higher success in pacing and cardioversion and shortens the time required to terminate an arrhythmia. Furthermore, optical mapping analysis allowed the visualization of spatio-temporal electrical waves on the heart surface. It could be shown that most arrhythmias were terminated by light stimulation of the excitable gap which caused the collision of the arrhythmic wave. The results obtained improve the understanding of optogenetic cardioversion from different perspectives and offer a head start in the design of experiments using large animal models with aims on a future clinical translation. 


\section{Contents}

1. Introduction

I. Scientific Background 3

2. The Heart 5

2.1. The electromechanical pump ................... 5

2.2. Electrophysiology of the heart .............. 6

2.2.1. The Cardiac Conduction System . . . . . . . . . . . . 6

2.2.2. The Cardiac Action Potential . . . . . . . . . . . . . . 8

3. Cardiac Arrhythmias 13

3.1. Arrhythmogenesis . . . . . . . . . . . . . . . . . . 13

3.1.1. Triggering events .................... 14

3.1.2. Heterogeneities or changes in the substrate (Reentry) . . . . . . . 15

3.2. Study of cardiac arrhythmias in isolated hearts . . . . . . . . . . . . 17

3.2.1. Langendorff perfusion . . . . . . . . . . . . . . . . 17

3.2.2. Optical Mapping . . . . . . . . . . . . . . . . . . 18

3.3. Arrhythmia treatment and the opportunity for optogenetics . . . . . . . . 19

4. Cardiac Optogenetics 21

4.1. Introduction . . . . . . . . . . . . . . . . . 21

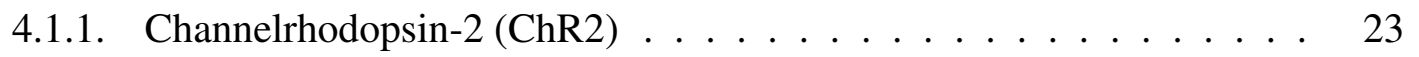

4.1.2. Why optogenetic control? .................... 23

4.2. Optogenetic control of cardiac electrophysiology ........... . . 24

4.3. Optogenetic arrhythmia termination . . . . . . . . . . . . . 27

4.3.1. First "optical shocks" in small animal models . . . . . . . . . . . . . 28

4.3.2. Mechanisms of optogenetic cardioversion . . . . . . . . . . 31

4.3.3. Determinants in optogenetic cardioversion . . . . . . . . . 32

5. Materials \& Methods 37

5.1. Light transmittance of the cardiac tissue . . . . . . . . . . . . . 37

5.2. Langendorff perfusion . . . . . . . . . . . . . . . . . . . . . . . . .

5.3. Investigation of pacing thresholds using optical fibers . . . . . . . . . 38

5.4. Pacing with different intensities, pulse lengths and surface areas . . . . . . 38 


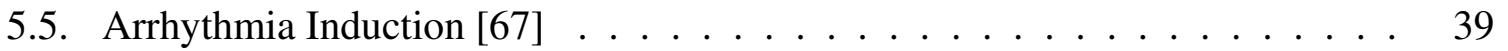

5.6. Global Illumination for Cardioversion [67] . . . . . . . . . . . . . . . . . . 39

5.7. Optical Mapping [67] . . . . . . . . . . . . . . . . . . . . . 40

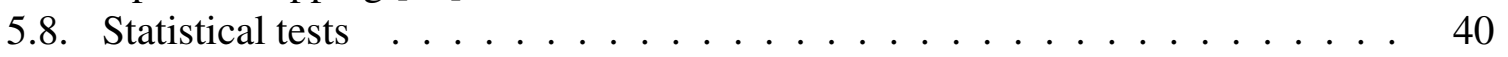

5.8.1. Unpaired t-tests . . . . . . . . . . . . . . . . . . 41

5.9. Determination of outliers for cardioversion times . . . . . . . 42

II. Results 43

6. Optogenetic Characterization of the ChR2 Mouse Heart 45

6.1. Introduction and Aim . . . . . . . . . . . . . . . . 45

6.2. Results . . . . . . . . . . . . . . . . . 47

6.2.1. Light Transmittance by the Murine Cardiac Tissue . . . . . . . . 47

6.2.2. The Effect of Di-4-ANBDQPQ and Blebbistatin on the pacing thresholds of the right and left ventricles . . . . . . . . . . . 51

6.2.3. Pulse length, intensity and surface area determine the optogenetic pacing threshold ..................... 52

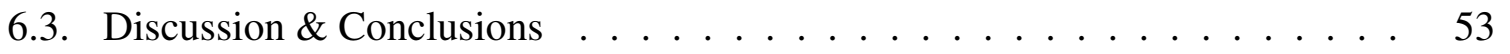

6.4. Contributions ....................... 55

7. Global Optogenetic Stimulation to Terminate Arrhythmias 57

7.1. Introduction and Aim . . . . . . . . . . . . . . 5 57

7.2. Experimental setup for global illumination . . . . . . . . . . . 58

7.3. Results (publication) . . . . . . . . . . . . . . . . 59

7.3.1. Global vs. Local Optogenetic Pacing . . . . . . . . . . . . . . 60 60

7.3.2. Global Optogenetic Cardioversion . . . . . . . . . . . . . 60

7.3.3. Effects of Global Illumination on Arrhythmia Patterns . . . . . . . . 60

7.4. Discussion \& Conclusions . . . . . . . . . . . . . . . 73

8. Optogenetic cardioversion based on arrhythmia cycle length and based on morphology

8.1. Introduction and Aim . . . . . . . . . . . . . . 75

8.2. Results . . . . . . . . . . . . . . . . . . . . 76

8.2.1. Illumination time based on arrhythmia cycle length . . . . . . . 76

8.2.2. Optogenetic Cardioversion of Monomorphic and Polymorphic Ventricular Tachycardia . . . . . . . . . . . . . . . . 78

8.3. Discussion \& Conclusions . . . . . . . . . . . . . . . . . . 80

9. Cardioversion times 83

9.1. Introduction and Aim . . . . . . . . . . . . . . . . . . . . . . . . . . . . .

9.2. Results . . . . . . . . . . . . . . . . . . . 83

9.2.1. Arrhythmia termination takes place in a specific range ...... 84 
9.2.2. Influence of illumination intensity and duration on the cardioversion time ...................... 86

9.2.3. Arrhythmic wave alteration after stimulation . . . . . . . . 87

9.3. Discussion \& Conclusions . . . . . . . . . . . . . . . . 90

9.4. Contributions ............................ 92

III. Discussion \& Conclusions 93

10. Discussion 95

11. Conclusions 97 


\section{Abbreviations}

AP Action Potential

APD Action Potential Duration

ChR2 Channelrhodopsin-2

LED Light Emitting Diode

LV Left Ventricle

MAP Monophasic Action Potential

ms milliseconds

MVT Monomorphic Ventricular Tachycardia

$\mathrm{mW} / \mathrm{mm}^{2}$ milliwatts per millimeter square 


\section{Introduction}

Covering cardiac electrophysiology, image analysis, physics and other fields, "Arrhythmia termination using Global Optogenetic Stimulation in ChR2 mice hearts" is a multidisciplinary work performed during my years of $\mathrm{PhD}$ training. The keywords in the title of this dissertation are heart, arrhythmias and optogenetics. Using these three words my $\mathrm{PhD}$ project can be explained as follows.

As we know the heart is the blood pump of the body and it functions via electrical impulses. An abnormal beating or function in the electrical propagation of the heart is called an arrhythmia. Electrical stimulation as given by pacemakers or defibrillators can force the heart to a normal rhythm. I used a new technique called optogenetics that allows stimulation of the heart using light as a harmless alternative to electrical stimulation. Naturally, hearts are not sensitive to light, thus transgenic mice hearts were used to perform experiments. These hearts expressed the light-sensitive ion channel Channelrhodopsin-2 (ChR2) that allows the photocontrol of the membrane potential of cardiomyocytes. Therefore the word "optogenetics".

Optogenetic cardioversion has been successfully demonstrated by different groups [12, 61, 19]. However, the mechanisms underlying optogenetic cardioversion remain largely elusive. In order to further understand the requirements and factors behind successful optogenetic arrhythmia termination I hypothesize that utilizing global epicardial illumination would lead to a decrease in both light intensity and pulse width necessaries to stop an arrhythmic activity on the heart and at the same time help us understand the events involved in this task.

The dissertation is divided into three parts, which are at the same time composed by chapters. The first part reviews the topics necessary to understand the work done. Here the reader can learn about the basics in order to understand the project, including the normal functioning of the heart, arrhythmias, the principles of optogenetics as well as its application on the cardiac field. The first part ends with a chapter describing the methods.

Part two constitutes the results; starting from the characterization of the response of the heart to optogenetic stimulation before moving into the investigation of a more complex task such as optogenetic cardioversion and lastly performing a deeper look into how optogenetic cardioversion works. Since each chapter has different research objectives, an introduction, results, discussion and conclusions section was included to each one. This way the reader can identify the corresponding meaningful information in a focused and accessible manner. Chapter 7 wraps the most important outcomes of my doctoral work in a published article 


\section{Introduction}

in Frontiers in Physiology. "Energy-Reduced Arrhythmia Termination Using Global Photostimulation in Optogenetic Murine Hearts" describes the first results obtained with the global stimulation setup I assembled with the advice of my supervisors. These first experiments paved the way for more interesting questions and experiments that are investigated in chapters 8 \& 9, such as the effects of arrhythmia morphology on cardioversion success and the time required to terminate arrhythimias using global illumination.

Lastly, part three closes the thesis with a general discussion of the project describing different outlooks and challenges emerging from this research work and the conclusions obtained. 


\section{Part I.}

\section{Scientific Background}





\section{The Heart}

\subsection{The electromechanical pump}

The human heart is a muscular organ composed of four chambers, working synchronously to pump oxygenated blood coming from the lungs into the circulation and non-oxygenated blood into the lungs coming from the system [24, 32].

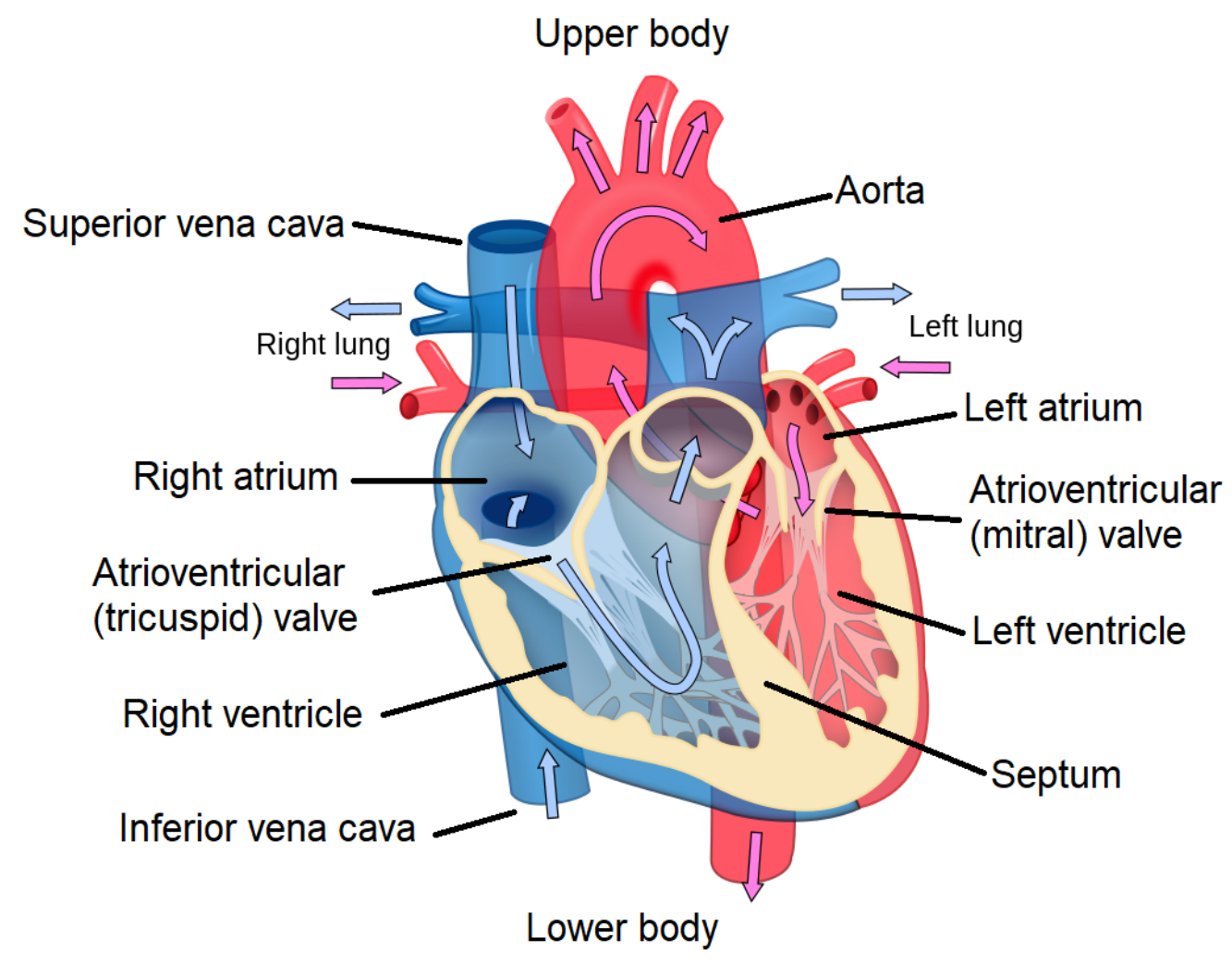

Figure 2.1.: Blood flow through the heart. Blood low on oxygen is transported from the systemic circulation and into the right atrium before being pumped from the right ventricle (RV) into the lungs. Freshly oxygenated blood returns to the heart through the pulmonary veins and into the left atrium. (Continues on next page.) 
Figure 2.1.: Then the atrium contracts pushing the blood into the left ventricle, which is the last location before its delivery back into the circulation ejected by the pressure formed due to the contraction of the ventricular walls. In summary, the right side of the heart is in charge of forcing the blood to the lungs for its oxygenation, and after that, the left side forces the blood to the rest of the body for its distribution. Obtained and modified from [99, 32].

The superior and inferior vena cava transport blood that is low in oxygen, coming from the upper body and from the trunk and legs, respectively. This blood flows into the right atrium, and then is pumped by the atrium through the tricuspid and into the right ventricle, which will be at the same time in charge of pumping it to the lungs. In the lungs, the blood will go through the process of oxygenation before returning to the heart through the pulmonary veins. It will reach the left atrium and then the left ventricle (LV). Due to the ventricle's contraction, the pressure will rise until it opens the aortic valve and finally gets ejected into the circulation [24, 32] (Fig,2.1).

The coordinated contraction and relaxation of the four chambers of the heart is ruled by its electrical activity. Therefore, arrhythmic behavior can lead to a variety of cardiovascular diseases. In order to understand the underlying mechanisms leading to the heart's function and malfunction it is important to know how electricity is generated and distributed in and around the heart from molecular to organ level [24, 32].

\subsection{Electrophysiology of the heart}

The heart is composed of millions of cardiomyocytes [48], specialized cardiac cells. Each cardiomyocyte is a living system on its own, composed of different proteins that synchronized generate changes on the voltage potential of the cell membrane. These changes lead to the contraction of the cell and at the same time to the ordered contraction of the chambers of the heart and ultimately to the pumping of the blood from the heart and into the body's supply system.

\subsubsection{The Cardiac Conduction System}

The generation and propagation of electrical signals in the heart follows a specific pathway in order to achieve a synchronous and ordered electrical excitation and further contraction of the heart. The atrial and ventricular myocytes, compose the major working force and also posses the ability to conduct electrical impulses. 
The cardiac conduction system is a network comprised of both pacemaker cells, which are in charge of periodically generating electrical signals [51], and conduction cells, which are responsible of transporting the signals (bundle of His, left and right bundle branches, and Purkinje fibers). This way the electrical excitation is generated and spread in a fast and highly ordered manner.

Figure 2.2 illustrates the cardiac conduction system. The signals originate in the sinoatrial (SA) node since it hosts the self-excitatory pacemaker cells. The SA node is located in the upper part of the right atrium. Therefore, the SA node and its cells are responsible for the heart beating frequency. A depolarizing current unique to these cells allows them to rhythmically generate action potentials. The electrical excitation generated by the action potentials is spread to the neighboring cells via specialized connections between cells, the gap junctions. The electrical wave travels first through right atrium and then through the left, contracting almost simultaneously. From there, the impulse is transported via junctional fibers to the atrioventricular (AV) node, which is located at the bottom of the right atrium, above the interventricular septum [34, 65, 81, 80].

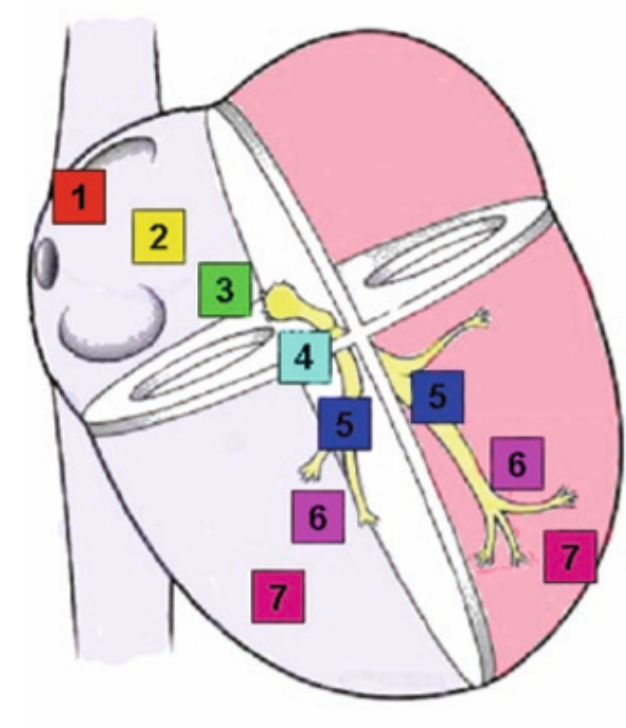

\begin{tabular}{|c|c|c|c|}
\hline $\begin{array}{c}\text { Normal } \\
\text { Activation } \\
\text { Sequence }\end{array}$ & Structure & $\begin{array}{c}\text { Conduction } \\
\text { velocity } \\
\text { (m/sec) }\end{array}$ & $\begin{array}{c}\text { Pacem aker } \\
\text { rate } \\
\text { (beats/min) }\end{array}$ \\
\hline 1 & SA node & $<0.01$ & $60-100$ \\
\hline 2 & $\begin{array}{c}\text { Atrial } \\
\text { myocardium }\end{array}$ & $1.0-12$ & None \\
\hline 3 & AV node & $0.02-0.05$ & $40-55$ \\
\hline 4 & $\begin{array}{c}\text { Bundle of } \\
\text { His }\end{array}$ & $1.2-2.0$ & $25-40$ \\
\hline 5 & $\begin{array}{l}\text { Bundle } \\
\text { branches }\end{array}$ & $2.0-4.0$ & $25-40$ \\
\hline 6 & $\begin{array}{l}\text { Purkinje } \\
\text { network }\end{array}$ & $2.0-4.0$ & $25-40$ \\
\hline 7 & $\begin{array}{l}\text { Ventricular } \\
\text { myocardium }\end{array}$ & $0.3-1.0$ & None \\
\hline
\end{tabular}

Figure 2.2.: The cardiac conduction system. The normal conduction starts at the sinoatrial node and excites the atria before reaching the atrioventricular node. After reaching the bundle of His, the pathway is branched into the right and left ventricles. The differences in the intrinsic pacemaker rates allow normal excitation to occur and if the SA becomes inactive, then the next structure on the activation pattern would generate the spontaneous rhythm. Image obtained with permission [81].

The main task of the atrioventricular node is to relay the electrical signal coming from the SA node to the ventricles. A delay in the signal transmission is also introduced through the $\mathrm{AV}$ junction, while conducting the impulses to the bundle of His. This delay has a functional 
purpose in the pumping action of the heart, allowing the atria to contract completely and empty the blood into the ventricles before these start to contract.

From the bundle of His the depolarization wave is bifurcated into the right and left bundle branches. The last transmission elements before reaching the ventricles are the Purkinje fibers, which conduct the impulse at a high speed and spread it to different points of the left and right ventricular walls. Lastly, the ventricular myocardial depolarization will occur from endocardium to epicardium and from apex to base, serving the pumping mechanics of the ventricles [34, 65, 81].

All of these electrical excitation events lead to a contraction, therefore the specific order and synchronization are crucial in order to pump the blood in an efficient and sustainable manner. However, synchronicity does not start at the structure level. The generation and propagation of electrical impulses and at the same time the contraction of the tissue start from activities at cell level. The next section is dedicated to understanding how an an electrical impulse generates.

\subsubsection{The Cardiac Action Potential}

\section{Membrane potential and ion channels}

Cardiomyocytes are electrically excitable cells. The selective permeability of their membrane keeps the inner and outer concentration of ions constantly at different levels, creating a transmembrane potential. The excitation of the membrane by electrical impulses changes its voltage. These changes lead to the opening of voltage-sensitive ion channels that allow a quick flow of different ions inside and outside the cell, altering the permeability of the cell and depolarizing it. Ultimately, the change in membrane potential is followed by the cell contraction. Membrane excitability of the cell will depend on different ion channels, pumps and transporters located through its membrane [32, 81]. In this section we will go through the most important actors and events that lead to the excitation and contraction of cardiomyocytes.

Ion channels are a group of protein molecules located in the cellular membrane. More specifically, cross the membrane from the outer part into the inner part in order to create a route of transport for ions and other specific molecules. Ion channels are distinguished by two important features: 1) They normally have a selective transportation, allowing only certain ions to travel across the membrane, and when they are open the ions move by diffusion along the channel due to the difference in concentrations of the specific ion inside and outside the cell. This kind of transportation along the membrane is defined as passive. 2) Many of these channels can open and close. These processes are regulated by either electrical signals (channels activated by a voltage threshold), or chemical signals (activated by ligands). The three types of ion channels that play the most important roles in cardiomyocyte electrophysiology 
are sodium, potassium and calcium, and they are all regulated by electrical signals [32, 81].

Myocardial cells hold a resting membrane potential of approximately $-90 \mathrm{mV}$. This potential results from the separation of anions and cations across the membrane and is mainly determined by the following factors: a) inner and outer concentration of charges (ions), b) permeability of those ions through the channels, and c) the activity of ion pumps.

Typically, in mammalian myocytes, the concentration of sodium ions $\left(\mathrm{Na}^{+}\right)$is higher outside the cell at approximately $140 \mathrm{mM}$ compared to an intracellular concentration of $5 \mathrm{mM}$ to $34 \mathrm{mM}$. In a similar way, calcium $\left(\mathrm{Ca}^{2+}\right)$ will have an extracellular concentration of approximately $3 \mathrm{mM}$, compared to an almost negligible amount inside the cell. Contrary to these cations, the concentration of potassium $\left(\mathrm{K}^{+}\right)$will be $104 \mathrm{mM}$ to $180 \mathrm{mM}$ intracellularly with just $5.4 \mathrm{mM}$ outside the membrane. These three ions play a critical role in maintaining the membrane potential at rest and also in inducing the changes in voltage during the action potential [34].

\section{Action Potential}

An action potential (AP) is triggered when the membrane potential is shifted towards a more positive value of approximately $-60 \mathrm{mV}$ due to a depolarizing current normally induced by neighboring cells through gap junctions. If this stimulus is not large enough to reach the threshold potential, no depolarization of the cell will occur and therefore, no action potential will be triggered [81].

If the threshold is reached, a series of opening and closing of different channels will take place, depolarizing and afterwards repolarizing the cell back to its resting potential. There are five phases identified during an action potential (Fig 2.3) [33, 81, 34, 59]:

Phase 0 - Rapid Depolarization: The stimulus that depolarizes the cell to the threshold activates the voltage-dependent sodium channels $\left(\mathrm{Na}_{\mathrm{v}}\right)$, abruptly changing the permeability of the membrane to $\mathrm{Na}^{+}$. Due to the difference in concentration, there is a rapid influx of $\mathrm{Na}^{+}$ into the myocyte depolarizing the cell to positive voltages of about $20 \mathrm{mV}$, almost exclusively generated by the movement of $\mathrm{Na}^{+}$cations. The voltage-gated $\mathrm{Na}^{+}$channels will inactivate within milliseconds from opening and the permeability of the membrane to $\mathrm{Na}^{+}$will then again be decreased. At the end of this phase and with a delay compared to the $\mathrm{Na}^{+}$channels, the voltage-sensitive $\mathrm{Ca}^{2+}$ channels will also start to open, increasing the permeability of $\mathrm{Ca}^{2+}$. At the same time the increased amount of intracellular calcium also induce the opening of $\mathrm{Ca}^{2+}$ channels inside the cell, at the sarcoplasmic reticulum. This process is called "calcium induced calcium release" and marks the beginning of the contraction process.

Phase 1- Early Repolarization: Together with the closing of the $\mathrm{Na}^{+}$channels, there is a small repolarization caused by the opening of $\mathrm{K}^{+}$channels, creating an outflow of $\mathrm{K}^{+}$ions and 


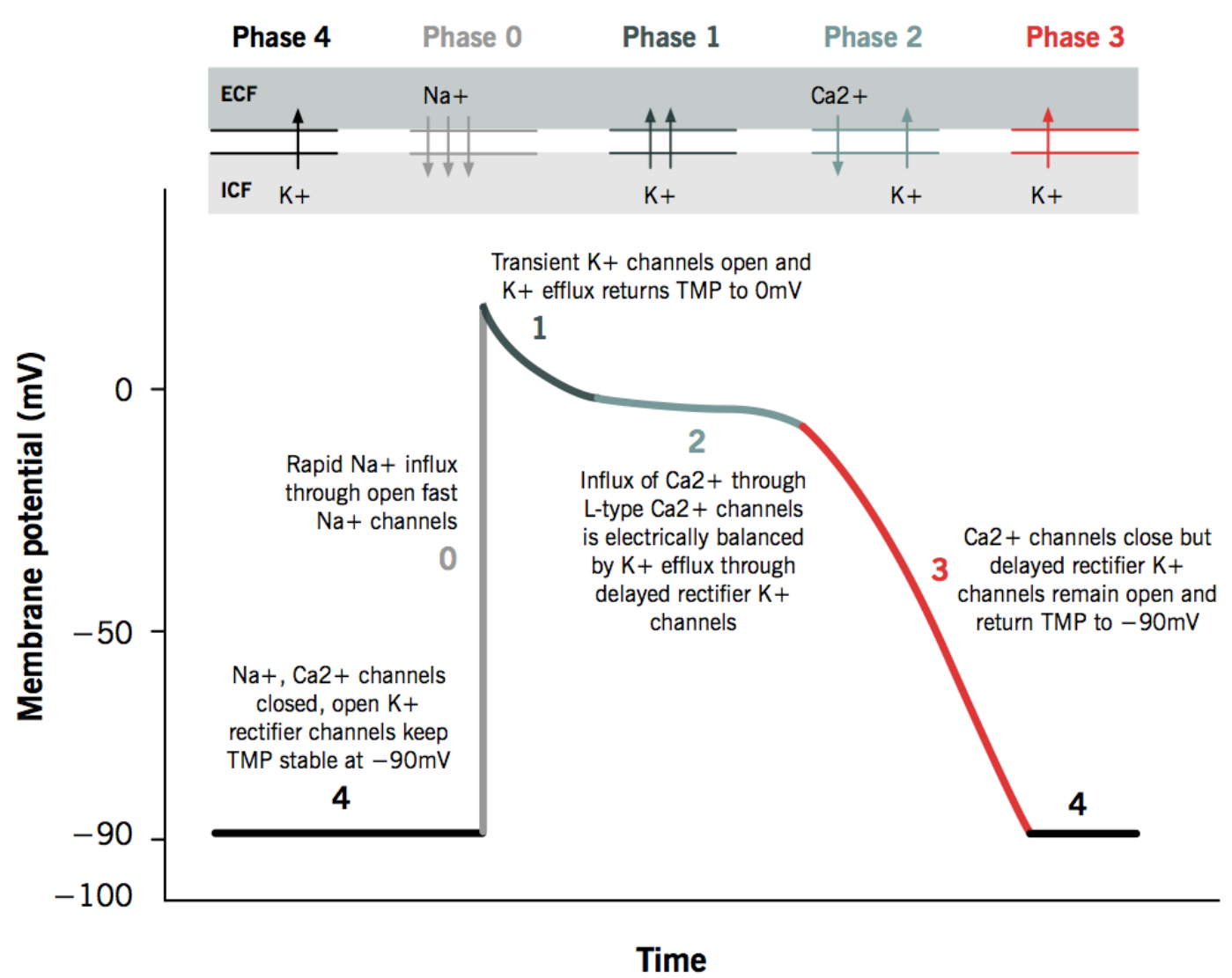

Figure 2.3.: Action potential of cardiomyocytes. The action potential is divided into five different phases, leading to the various changes in the transmembrane potential (TMP). Phase 0 is characterized by the rapid depolarization caused by the opening of voltage-dependent sodium channels. In Phase 1, a small repolarization takes place due to the efflux of potassium before the plateau phase (Phase 2) where a balance of inward $\mathrm{Ca}^{2+}$ current and $\mathrm{K}^{+}$outward currents keeps the voltage stable. At last, $\mathrm{Ca}^{2+}$ channels close in Phase 3, leading to a repolarization back to negative values by the continuous $\mathrm{K}^{+}$currents. During Phase 4 the membrane potential is stable at the resting state until another stimulus depolarizes the membrane up to the threshold. Image obtained from [35].

reducing the membrane potential to approximately $0 \mathrm{mV}$. Inactivation of the $\mathrm{Na}^{+}$channels can happen as soon as $1 \mathrm{~ms}$ after their opening and excitation of the cell will not be possible until the majority of the channels recover. Therefore, these channels are the main determinants of the excitability of the cells of each specific region of the heart.

Phase 2 - The plateau phase: Due to the outflow of potassium and the inflow of calcium through L-type calcium channels, a plateau phase is reached, where the voltage is stable around $0 \mathrm{mV}$ for a period of a couple of hundreds of milliseconds. L-type calcium chan- 
nels inactivate at a slower pace compared to other voltage activated channels such as the $\mathrm{Na}_{\mathrm{v}}$ and are therefore important contributors to the plateau phase. As mentioned in Phase 1, the influx of $\mathrm{Ca}^{2+}$ triggers the release of $\mathrm{Ca}^{2+}$ from intracellular compartments, leading to the contraction of the cell.

Phase 3 - Repolarization: This phase ends the action potential. The gradual inactivation of $\mathrm{Ca}^{2+}$ channels, together with the $\mathrm{K}^{+}$outward currents bring the membrane potential back to its negative values. Moreover, the activity of different ion pumps returns the sodium and calcium ions outside of the cell and potassium ions to the interior.

Phase 4 - The Resting Phase: This is the original phase of the cell before receiving a new stimulus and triggering an action potential again. In this phase $\mathrm{Na}^{+}$and $\mathrm{Ca}^{2+}$ channels are closed and a different $\mathrm{K}^{+}$current helps maintaining the negative membrane potential. However, $\mathrm{Na}_{\mathrm{v}}$ channels will need time to recover before gaining the ability to be excited again. This time is called refractory period.

Therefore, during the refractory period cells cannot be excited. It is the interval of time from depolarization to recovery of excitability, and it is related to the action potential duration. Differences in the refractory period in adjacent regions can aid the generation of arrhythmia [14]. Arrhythmia and arrhythmogenesis will be explained in Chapter 3 .

The expression of ion channels as well as their properties will be different for each structure of the heart (sinoatrial node, atrioventricular node, atria, ventricles) and will also vary for different animal species. Therefore, cardiac action potentials have different waveforms depending on their location. Those differences will contribute to the normal propagation of excitation waves through the heart. Fig 2.4 A illustrates the waveforms for the excitation pathway described before.

Fig 2.4 B \& C show the differences between human and mouse action potentials. The latter is shorter and is lacking a plateau phase. These two characteristics can be determined by a smaller expression of $\mathrm{Ca}^{2+}$ channels on the cell membrane compared to the human heart and by a larger expression of $\mathrm{K}^{+}$. The differences lead to a faster repolarization of the membrane potential and shorter action potentials (30-80 milliseconds [ms] compared to $150 \mathrm{~ms}$ to $400 \mathrm{~ms})$. 


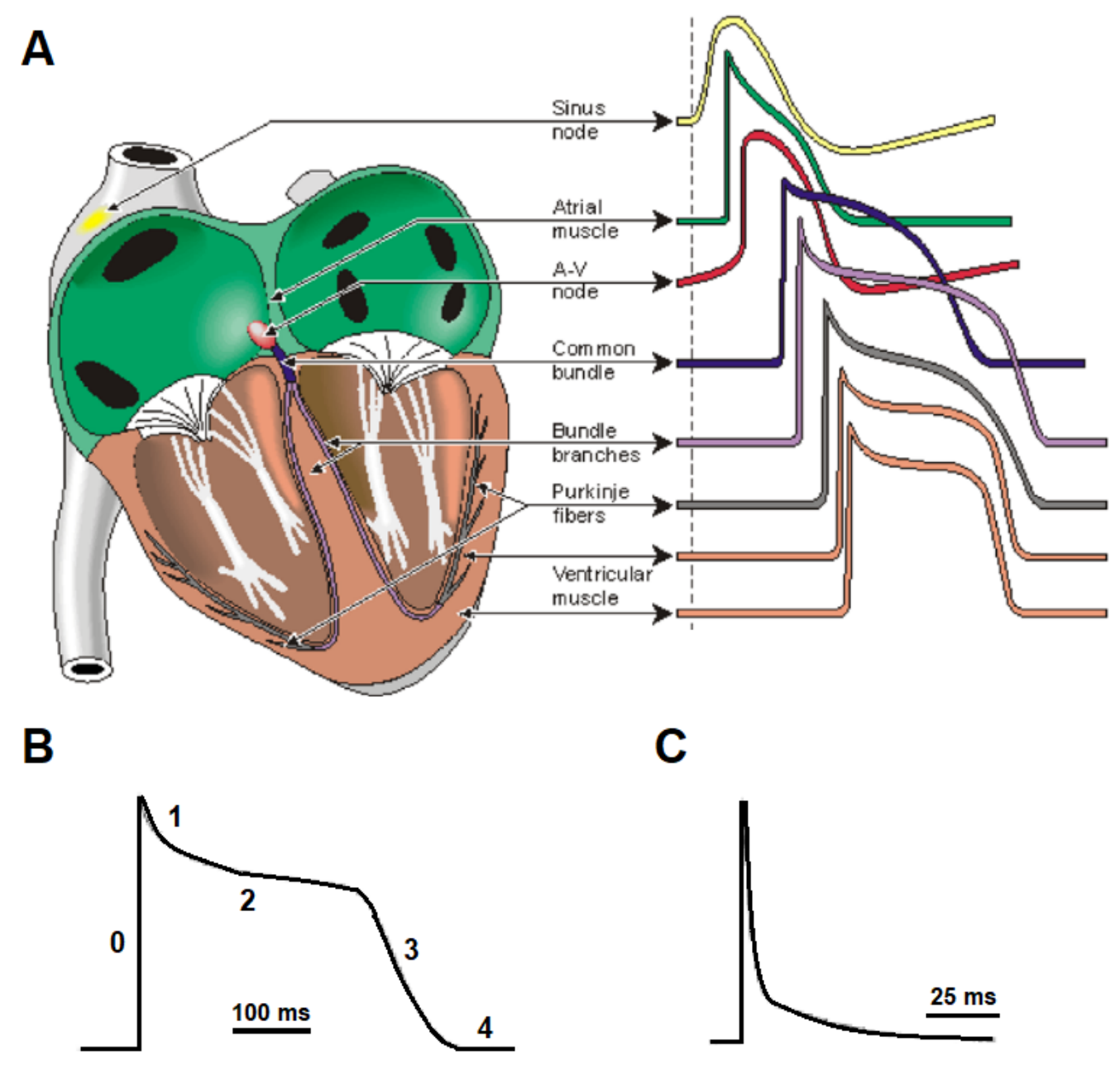

Figure 2.4.: Differences in action potentials. (A)Regional differences in the action potential waveform, and how the regional electrical signals form up the electrocardiogram (ECG). (B)Human and (C) mice action potential waveform. Images obtained and modified with permission from [59, 33]. 


\section{Cardiac Arrhythmias}

Cardiac arrhythmias are defined as an abnormal rhythm of the heart, which in other words would be abnormal electrical activity in the heart. As discussed in Chapter 2, the heart follows an ordered electrical excitation automatically starting at the sinoatrial node and ending at the ventricular myocardium. A disruption of the frequency, origin or route of the electrical propagation can cause or be considered an arrhythmia.

There exists a variety of types of arrhythmias, with also different ways to classify them. For example by heart rhythm, where a bradycardia is termed for a slow heart rate, tachycardia for an abnormally fast heart rate, and during fibrillation the pace, amplitude and morphology are not constant [81, 72]. Arrhythmias can also be classified depending on the region of the heart where they take place. Supraventricular tachycardia originate in the atria and ventricular arrhythmia in the ventricles. An additional classification is by the morphology they show in an electrical recording such as the electrocardiogram, separating them into monomorphic and polymorphic arrhythmias [82].

When the heart is suffering an arrhythmia, it is unable to properly pump blood from its chambers and into the circulation. Sudden cardiac death is one the leading causes of natural deaths in the United States and Europe and approximately half of them are attributed to ventricular arrhythmia [37].

\subsection{Arrhythmogenesis}

A healthy heart is able to maintain its function without any anomalies. Arrhythmias indicate abnormal electrical activity of the heart. The causes of an arrhythmia are broad and they commonly represent a multiscale problem that can originate from one or many of the different scales the heart functions: molecular, cellular, tissue, structures, chambers. Alterations in function of the ion channels can promote arrhythmia initiation, and so can changes in the conductivity of a specific region of the ventricle caused by ischaemia. Nonetheless, the abnormal electrical activity, leading to the initiation of arrhythmias can present in two forms: a) abnormal impulse generation which are triggering events, or b) abnormal impulse conduction, such as heterogeneities or changes in the substrate that affect the pathway of conduction and can lead to reentry. Both of these mechanisms on their own or combined can provoke the 


\section{Cardiac Arrhythmias}

initiation of arrhythmias [66, 39, 23, 33]. In this section we will look into the mechanisms and focus on ventricular arrhythmias.

\subsubsection{Triggering events}

One of the most common mechanisms that trigger arrhythmias at cellular level are afterdepolarizations (Fig. 3.1), which are abnormal impulses or extrasystolic membrane depolarizations (outside of the normal depolarization) that can lead to action potentials. These action potentials at the same time can ignite ectopic beats which may result in sustained tachycardia.
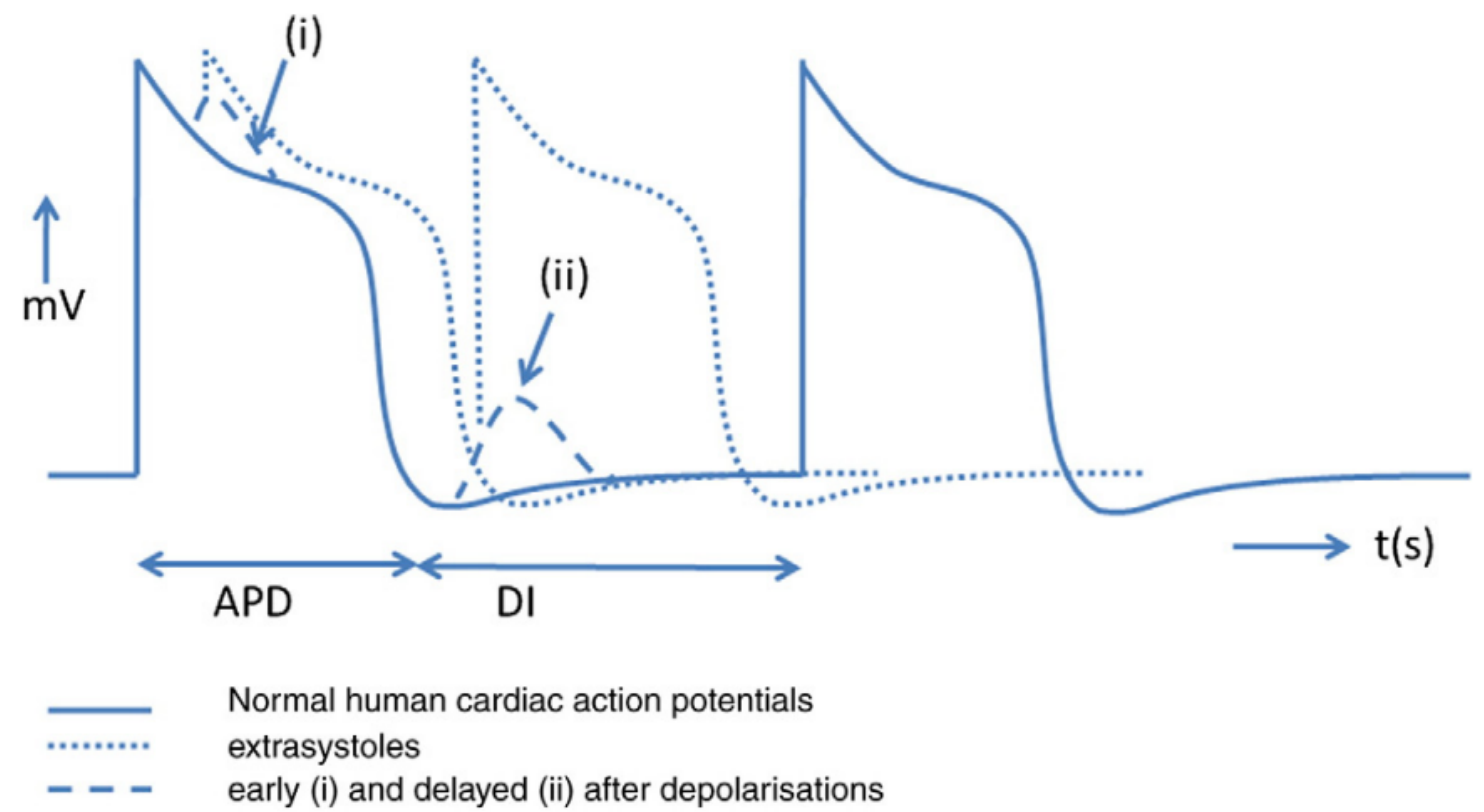

Figure 3.1.: Early after-depolarizations and delayed after-depolarizations. EADs take place during the repolarization (i) of an action potential, while DEAs arise once the cell is again repolarized (ii). They can both prompt extrasystoles. Image obtained with permission from [39].

Early after-depolarizations (EADs) occur during the repolarization time of a prolonged action potential (Phases 2-3). This allows the L-type $\mathrm{Ca}^{2+}$ channels to recover. As mentioned before, these channels are normally open during Phase 2 and are responsible of the plateau in the action potential. The delayed repolarization is commonly caused by a decreased outward potassium current, and if it enables enough time $\mathrm{Ca}^{2+}$ channels recover their excitability, reopen and produce a depolarizing current. This at the same time initiates a positive feedback on channel opening resulting in the after-depolarization and potentially triggering an action potential [66, 39, 23, 33]. 
Delayed after-depolarizations (DEAs), different from EADs, occur after a full repolarization (Phase 4). They can be elicited from an enhanced $\mathrm{Ca}^{2+}$ release from the sarcoplasmic reticulum [66, 39, 23, 33].

Other examples of triggering events are alternans and automaticity. During alternans, cells present changes in the action potential duration, alternating long and short action potentials. Automaticity is when cells that normally do not depolarize on their own do so. Pacemaker cells of the sinoatrial node are able to periodically undergo spontaneous depolarization, and this is normal automaticity. Enhanced automaticity (increased rate), or automaticity acquired by other cells that are not functioning as pacemaker cells can lead to arrhythmia generation [82].

\subsubsection{Heterogeneities or changes in the substrate (Reentry)}

Tissue heterogeneities can lead to arrhythmias in different manners, and they are the basis of most arrhythmias in the clinic. Reentry refers to a circulating electrical wave in which an impulse travels repetitively around a region of the heart. The prerequisites for the development of reentry are: 1) an abnormal electrical circuit around an anatomical block, 2) an unidirectional block and 3) conduction velocity slow enough to allow recovery of excitability in time for reexcitation by the circulating impulse. The wavelength is defined as the product of the effective refractory period by the conduction velocity of excitable media such as cardiac tissue. If a on of the paths in a circuit bifurcation presents a decrease in wavelength an excitation loop in this circuit can be created. The path with lower conduction velocity forms a loop if the propagation is first blocked due to a longer effective refractory period, then a next wave could travel retrogradely along the path that was originally unexcitable creating the reentry circuit (Fig $3.2 \mathrm{~A}$ ). This is called anatomical reentry and many times can give rise to a monomorphic ventricular tachycardia (MVT) [23, 66, 14].

An arrhythmia can also be triggered without an anatomical obstacle. Slowed conduction velocity due to a decreased membrane excitability or decreased cell-to-cell coupling may also result in slowed or blocked conduction. In a different scenario, a heterogeneous area on the tissue with a longer refractory period can also initiate an arrhythmia. An electrical wave originating in the periphery would not propagate through this specific area, but instead travel around it. Afterwards entering it from the opposite direction creating what is called a figure of eight reentry (Fig 3.2 B). In yet a different scenario, a region of cells with triggering activity surrounded by unequal dispersion of refractoriness could start a propagating wave only towards one direction but not to the other initiating a reentrant activity (Fig.3.2 C) [39, 14]. 
A

A Sinus Rhythm

1
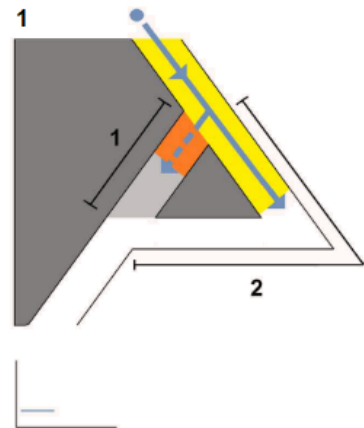

Re-entry Circuit
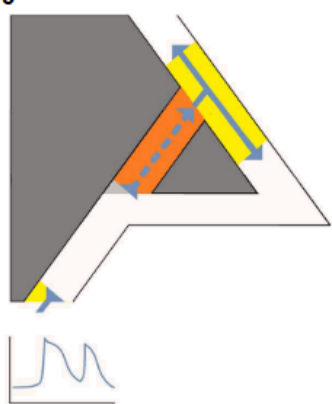

B

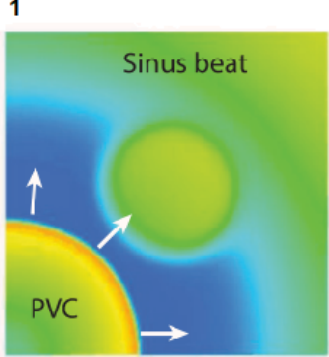

$\mathbf{C}_{1}$

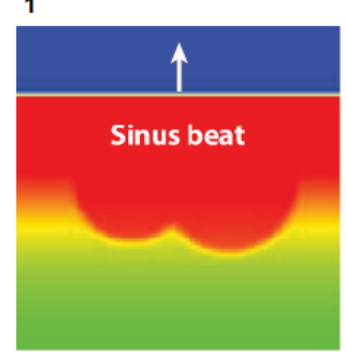

2

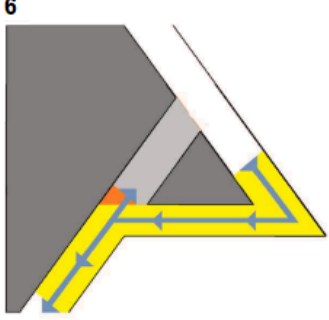

\section{Noum \\ Re-entrant tachycardia}
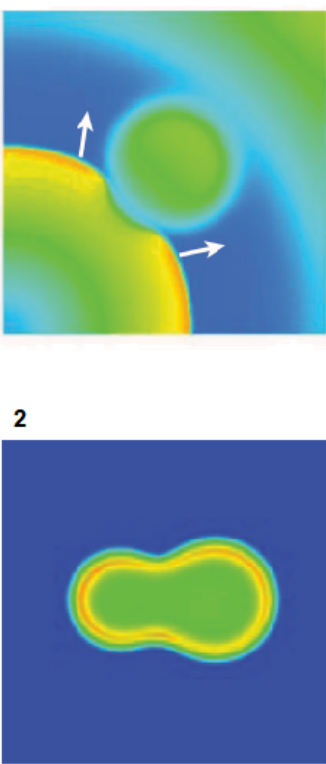

Triggered activity

3

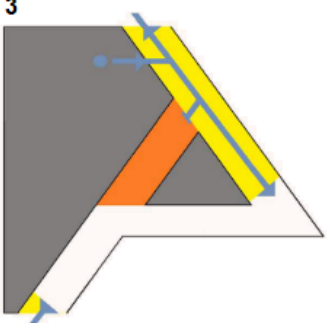

$\Lambda$

Electrical impulse

$\square$ Non-conducting myocardium

$\square$ Slow conducting myocardium

$\square$ Normal myocardium

$\square$ Refractory myocardium

$\square$ Myocardium with increased refractoriness

$\boldsymbol{\theta} \times \mathrm{ERP}=$ Wavelength of excitation $(\lambda)$

If $\lambda<$ dimensions of the circuit then re-entry

3

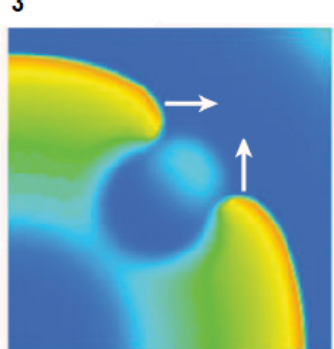

3
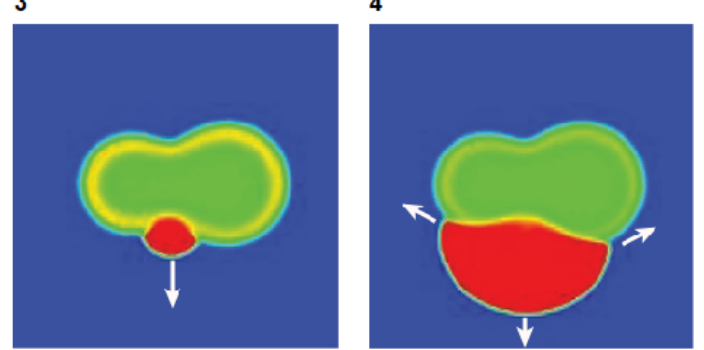

$-80 \mathrm{mV}$

Voltage
4

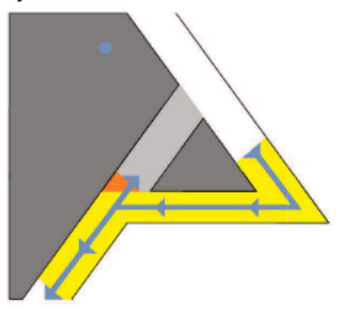

Ectopic AP

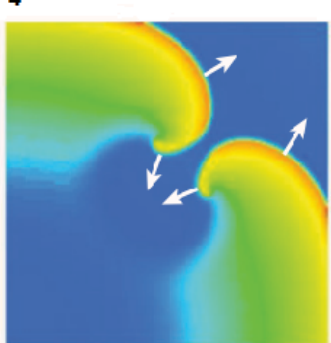

$10 \mathrm{mV}$

Figure 3.2.: Arrhythmogenesis due to heterogeneities in the cardiac tissue. Caption on next page. 
Figure 3.2.: Arrhythmogenesis due to heterogeneities in the cardiac tissue. (A) An anatomical reentry can be initiated when an anatomical block separates two pathways with different conduction velocities. After a wave originated by the sinus rhythm propagates across the two pathways $(1,2)$ a second wave coming from an abnormal activity or source will not be able to travel through pathway 1 (light gray), since it has an increased refractoriness (3). Once the wave reaches the end of pathway 2 (white) it will be able to travel retrogradely $(4,5)$ through pathway 1 initiating the re-entrant arrhythmia $(5,6)$. (B) A region with longer refractory period can also initiate arrhythmia reentry when an unidirectional conduction block occurs in this region. An ectopic wave (white arrows) traveling after a sinus wave (1) would travel around the region presenting the heterogeneity $(2,3)$ and then retrogradely into it (4) starting the arrhythmic pattern. (C) Reentry can also be induced when the same region of the cardiac tissue presents abnormal electrical activity (such as early afterdepolarizations, in this case) and heterogeneities in the propagation. After a normal excitation, an ectopic activity due to EAD would first excite the area with highest conductivity and then propagate. Images obtained and modified with permission from [44, 66]

Even though the mechanisms described before are the most common, there are other mechanisms based on the same principles that can start arrhythmias. In summary, a combination or one of the following is needed: a) an ectopic electrical activity or trigger, b) heterogeneities in the substrate such as a conduction block or differences in the effective refractory period or conduction velocity.

\subsection{Study of cardiac arrhythmias in isolated hearts}

\subsubsection{Langendorff perfusion}

The Langendorff perfusion is a technique to reanimate isolated hearts in vitro. It has been used to investigate a number of different physiological phenomena in the heart such as electrical activity, blood flow regulation, metabolism, effects of drugs, and others. One of the advantages of the technique are the study of the isolated heart without being influenced by other organs and systems from the body. This of course at the same time makes the results obtained harder to directly translate into a clinical scenario [5].

During the experiment the hearts are retrogadely perfused by cannulating the aorta, with the pressure of the perfusate closing the aorta and allowing it to flow through the coronary system [81]. This is an advantage especially for the study of cardiac arrhythmias since the electrical malfunction investigated will not lead to a decrease in perfusion of the heart since it 


\section{Cardiac Arrhythmias}

will not depend on its own pumping to be perfused.

The heart is suspended in a prewarmed bath to keep it at optimal temperature. The perfusion flow can be controlled either via constant pressure or constant flow and during the experiment this and other physiological parameters such as the temperature, $\mathrm{pH}$ and electrical activity can be measured to keep track of the heart's status. In order to keep the heart close to a physiological condition, the perfusate is oxygenated with $95 \%$ oxygen and $5 \% \mathrm{CO}_{2}$ and heated up to the necessary estimated temperature [5].

Once the perfusion setup is running it can be adapted to the specific needs of the study. Since in this PhD work I am looking to understand cardiac arrhythmias, the optical mapping technique was also implemented for some experiments.

\subsubsection{Optical Mapping}

Optical mapping is a technique based on optical sensors which can be fluorescent dyes or genetically encoded and are used to track the transmembrane potential or intracellular calcium concentration of isolated hearts or 2D cardiac cultures. It is a contactless method that has improved the study and understanding of different electrophysiological phenomena such as cardiac arrhythmias. This is due to its ability to record action potentials from different regions of the heart simultaneously that result in the spatiotemporal mapping of travelling waves across the sample [63, 81].

In order to perform optical mapping of transmembrane voltage (implemented for this doctoral thesis), the fluorescent voltage sensor is loaded into the sample. Synthetic voltage sensors can be directly injected to the hearts using the Langendorff perfusion technique. Once the dye is loaded, a source light with the excitation wavelength is used to illuminate the sample, resulting in the emission of photons of a characteristic wavelength that can be detected by the sensor and further analyzed. In order to obtain optimal signals of the phenomena studied, a proper selection of excitation and emission filters as well as detectors with the spatial and temporal resolution needed must be chosen [63, 81].

Chapter7 discusses the setup designed from a Langendorff perfusion system adapted to obtain optical mapping measurements of cardiac optogenetic experiments for this project and in Chapter 5 the technical details are also described. In optogenetics, the heart is stimulated by light. Therefore the setup is capable of stimulating the heart at certain wavelength and exciting the voltage-sensitive dye at different wavelength. Cardiac optogenetics will be described in detail in the following chapter. 


\subsection{Arrhythmia treatment and the opportunity for optogenetics}

Treatments will depend on the type of arrhythmia the patient is presenting, but there are generally three types of treatments: electric therapy, antiarrhythmic drugs and ablation [31, 37]. Antiarrhythmic drugs are mainly used in the cases of recurrent symptomatic arrhythmias. Examples are beta blockers and ion channel blockers. However, in some cases antiarrhythmic drugs can worsen arrhythmias and by having proarrhythmic effects or result toxic for the patient [37, 16]. Electrical therapy, in the case of an implantable cardioverter-defibrillator, aids in the prevention of sudden cardiac death and can decrease the mortality of high-risk patients. Nonetheless, there is a risk of an inappropriate shocks being delivered [8]. And shocks (appropriate or inappropriate) are not free of risks. They can cause unbearable pain, damage to the myocardial tissue, psychological discomfort and increase the mortality of the patient [7, 52, 47, 37].

Optogenetics is a biological technique that enables light-induced depolarization of cells, and therefore has a great potential in the treatment of arrhythmias. Even though its use in the clinics still has different hurdles to face [69], the technique can be used as a tool to investigate the initiation and termination of arrhythmias at a spatiotemporal resolution uncomparable to the resolution offered by drugs or electrical therapy and with the promise of non-harming control of cardiac tissue. The next chapter is focused on describing optogenetics in the cardiac field. From the first findings in single cells and cell cultures to optogenetic cardioversion and its mechanisms. 



\section{Cardiac Optogenetics}

\subsection{Introduction}

The word "optogenentic" was introduced by Deisseroth in 2006 as a combination of optics, genetics and bioengineering in order to control and study neural circuits [22]. Nowadays, optogenetics is used to control varied biological mechanisms through the expression of light-sensitive proteins.

In optogenetics, light sensitivity can be inscribed in cells and tissues, originally in the form of ion channels and pumps. However, the optogenetics toolbox now includes diverse proteins that with a shine of light of a specific wavelength can be used to control different cellular and sub-cellular functions [90, 49, 76]. Potential applications of optogenetics lie in neuroscience, oncotherapy, cardiovascular diseases, diabetes therapy, gene editing and other medical fields [95].

There are four main steps in order to apply optogenetics as a method to study living organisms [64]:

1. Selection or creation of the light activated proteins. The first channels to be tested were directly taken from algae. However, the advances in bioengineering have led to creation and modification of different channels and other proteins. Regarding ion channels, the range of applications has been amplified thanks to the manipulation of their properties. Some of these are wavelength sensitivity, peak and steady current, delay from stimulation to activation, recovery time after activation and the sensitivity of photocurrent to changes in light intensity among others [55]. A clear example is ChR2-H134R -a mutant of ChR2 with greater steady current- which is most commonly used in the cardiac optogenetics field [58].

2. Gene delivery. There are mainly three methods to photo-sensitize cells; transfection, viral transduction or creating transgenic animals. A promoter can be used when applying viral therapy, giving optogenetics yet another possibility to make it cell or tissue specific. However, potential translation into the clinic represents a challenge and the most promising alternative at the moment appears to be viral gene delivery, which is still questionable for different safety reasons. 


\section{Cardiac Optogenetics}

3. Illumination. One of the key advantages of optogenetics compared to other methods of stimulation such as electrical or drugs is the spatio-temporal precision offered by light. Illumination technologies can be at the same time the limiting factor and the facilitator. The illumination source should depend on the type of control needed to be achieved. From optical fibers [96], lasers, LEDs ([67]), micro-mirror devices ([19, 77, 50]) to microscopes [12], and micro-LEDs embedded in a 3D multifunctional integumentary membrane [94] each one will vary on spatio-temporal resolution, light-crafting capabilities and amount of energy delivered. Optical control of living systems such as the heart or cardiomyocytes offers a new level of interrogation capabilities that were impossible to achieve using electrical or drug stimulation.
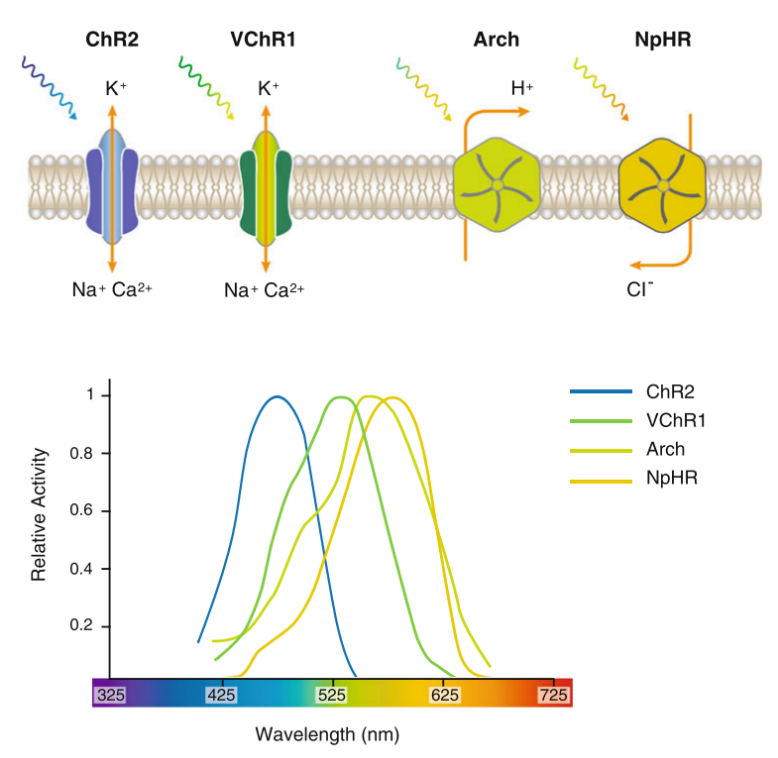

Figure 4.1.: Optogenetic tools controlling membrane potential. The first applications of optogenetics were focused on changing the membrane potential of cells. (A) Ion channels such as Channelrhodopsin-2 (ChR2) and Channelrhodopsin-1 (VChR1) have been mainly used to depolarize the cells by allowing the entrance of cations, while ion pumps such as archaerhodopsin-3 (Arch) and NpHR hyperpolarize the membrane by either pumping cations to the exterior or anions to the interior of the cell. Moreover, these proteins are sensitive to (B) different wavelengths, therefore depolarizing and hyperpolarizing proteins could be combined to achieve a finer control of the membrane potential. Image obtained and modified with permission from [68].

4. Reading and interpreting the outcome. Some often used examples in the case of cardiac optogenetics can be the action potential during a patch clamp experiment, electrical signal such as the ECG using electrodes, or topological information of a monolayer or an isolated heart using optical mapping. The analysis of one or several outcomes will help better understand the electrophysiological behaviour of the heart. 
Since this doctoral thesis is focused on the understanding of optogenetic arrhythmia termination via global illumination, we will mainly focus on steps 3) and 4). For the first step it is of importance to understand the kinetics of the ion channel used in our model, Channelrhodopsin-2 (ChR2), and for the second step that we are using a $\alpha$-MHC-ChR2 transgenic mouse model, which expresses $\mathrm{ChR} 2$ in $>90 \%$ of its cardiomyocytes.

\subsubsection{Channelrhodopsin-2 (ChR2)}

The identification of Channelrhodopsin-2 in the green algae Chlamydomonas reinhardtii and its further cloning and characterization triggered the field of optogenetics as a technique to control excitable cells via light sensitive ion channels [57]. So far it has been the most important tool to study both neural and cardiac activity without the need of electrical or drug stimulation and it is also the optogenetic tool used in this doctoral work.

ChR2 is a non-selective cation channel with its highest affinity to $\mathrm{H}^{+}, \mathrm{Na}^{+}, \mathrm{K}^{+}$and $\mathrm{Ca}_{2}{ }^{+}$. Once it is illuminated, the opening of the channel will lead to a fast rise of its current (in approximately $200 \mu \mathrm{s}$ ). ChR 2 uses retinal as its photosensing element. Since it is a passive membrane transport protein dependent on the electrochemical gradients, the current elicited with its opening will depend on the membrane potential.

Two different characteristic currents can be perceived; the peak current, reached on the first micro to milliseconds from the moment of illumination, and a steady-current which will have a lower amplitude and can be sustained for longer. The peak current of channel will also have a recovery time, therefore if a second light pulse is used to stimulate it, the current will depend on the time elapsed, and a full recovery can take up to several seconds something that will not affect the steady current coming afterwards. Closing of the channel will take approximately $10 \mathrm{~ms}$ to $20 \mathrm{~ms}$. [57, 11, 26, 78].

\subsubsection{Why optogenetic control?}

Genetic targeting presents the possibility of discriminating the rest of the different cells and tissue that is not subject of optogenetic control or study. Light stimulation enhances even further the capabilities of spatial and temporal resolution and precision. With these new tools, a variety of scales can be chosen to control and study.

Moreover, electrical stimulation imposes an injection of current that affects the cell or tissue without considering the current electrophyiological condition of the target. An effect provided by an optogenetic stimulus will always be modulated by the ionic balances in and outside the cell, as well as by the other cells surrounding it, providing a sort-of feedback response that will manipulate the amplitude of the photo-current injected. Optogenetic control 


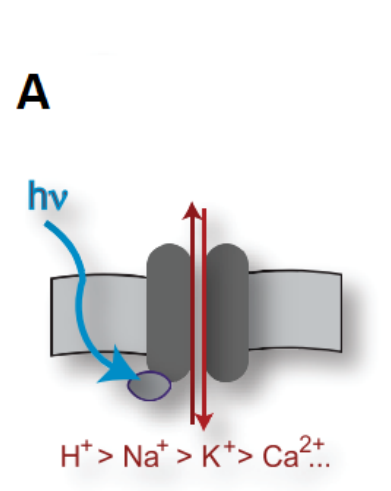

B
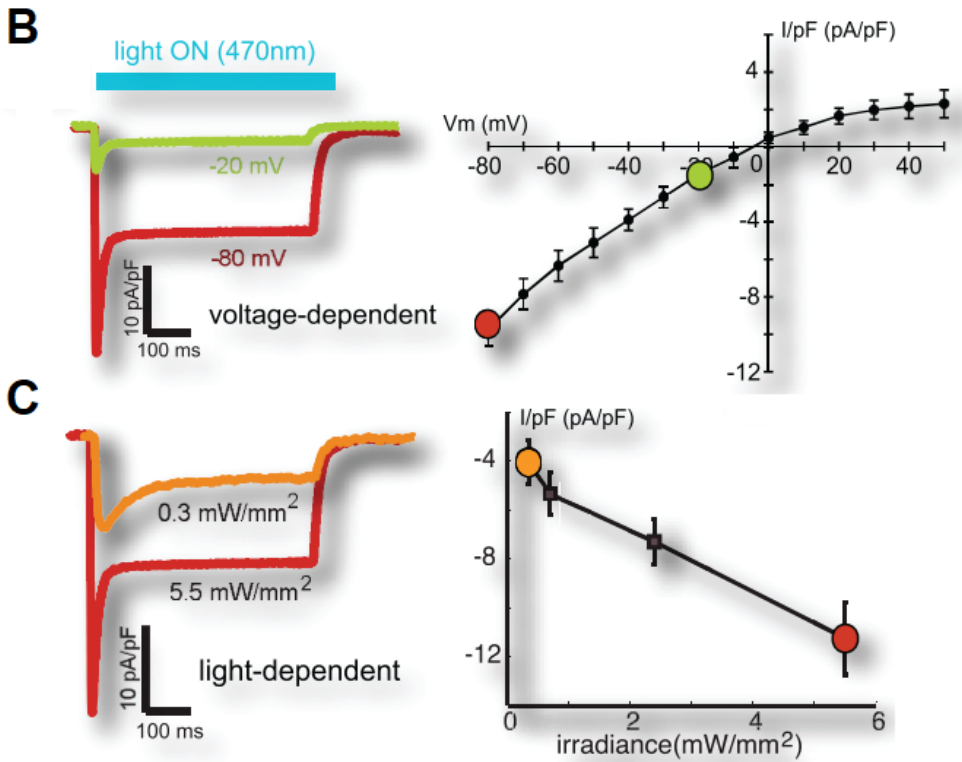

Figure 4.2.: Properties of Channelrhodopsin-2. (A)Channelrhodopsin-2 allows cations to flow with different affinity. The photo-current elicited is both (B) voltagedependent and $(\mathbf{C})$ light-dependent. When the membrane potential rests at greater negative value the current will be larger. Image obtained and modified with permission from [26]

generates bio-current, compared to super imposed electrical current that could lead to damage of the cells.

\subsection{Optogenetic control of cardiac electrophysiology}

The spatio-temporal control of the electrophysiology of the heart using optogenetics has been demonstrated with high precision at different scales. From milliseconds to seconds, cell level to organ level as well as different light intensities and illumination patterns, having an overview of all the capabilities in optogenetic control is of great importance to understand the outcome of such stimuli and to find an optimal solution for the control and termination of arrhythmia in the light-sensitive heart. It is well known that optogenetics offers high spatiotemporal precision but it is also important to consider that light intensity provides yet another dimension since different amounts of light will lead to different responses.

In one of the first computational models of cardiac optogenetics Abilez and colleagues [1] validated with experiments the ability to pace cardiomyocytes via optical stimulation at different frequencies and the effect of the light intensity on the photocurrent injected into the 
cells. When investigated using patch clamp, Channelrhodopsin-2 (ChR2) current reaches a peak during the first $20 \mathrm{~ms}$ of illumination and later decays to a steady-state current, both dependent on the intensity of the pulse applied. In a similar manner, lower intensities will result in longer delays of the action potential generation [11, 1]. Stimulating in a precise timecontrolled fashion, optogenetic stimulation during the generation of an action potential can shape its morphology depending on the delay from the beginning of the action potential [92].

Optogenetic pacing of ChR2-expressing hearts will also lead to a 1:1 capture of the cardiac tissue, and for shorter pulses a higher amount of light will be required [11]. Moreover, the amount of energy needed to pace on the ventricles will be lower than in the atria [11], with the right ventricle being sensitive to the least amount of light [96]. In vivo spatial precision was corroborated using optical fibers to stimulate specific regions of the heart that were followed by varied responses depending on the stimulation place of ChR2 mice hearts [96, 17] (Fig. 4.6 E). In the same study, one more of the advantages of optogenetics was employed, as specific transgenic expression of ChR2 in either cardiomyocytes or the purkinje fibers was used to investigate the differences and susceptibilities of arrhythmia triggers between the two cell types and specific regions of the heart, proving that extrasystoles and ectopies can be generated by stimulating approximately ten fold less purkinje fibers than cardiomyocytes.

High spatio-temporal control in cardiac monolayers has seen further enhancement. Aided by technological advances, in this case a digital-micromirror-device (DMD), light was crafted in order to achieve precise control of excitation waves [15]. Direction of the waves was controlled by a synchronized series of light stimuli, first creating a one-sided conduction block with a long, high intensity pulse and during the period of refractoriness applying a next short low intensity pulse to generate a wave that would only travel the opposite direction of the block. Control of conduction velocity of the traveling waves was acquired by benefiting from the dimension offered by the manipulation of the light intensity. By applying a sub-threshold pulse (light at intensities that will not lead to an activation wave), cells' membranes were brought close to the excitation point, therefore increasing the conduction velocity once a second light pulse triggering propagation was applied. Lastly, by imposing a spiral shaped light stimulus with opposite chirality, spiral wave chirality manipulation was achieved for the first time in excitable media.

At the moment of planning and designing optogenetic control, it is also of importance to consider the delivery method and how each one of these can lead to different spatial distributions of the light-sensitive cells, and at the same time, which kind of illumination is better suited to the distribution obtained [9].

The knowledge of all these features in optogenetic control of cardiac electrophysiology paved the way first to understand the mechanisms that can be used to terminate arrhythmias, and then to optimize arrhythmia control. The different methods and bases of optogenetic arrhythmia termination are described in the next section. 
A
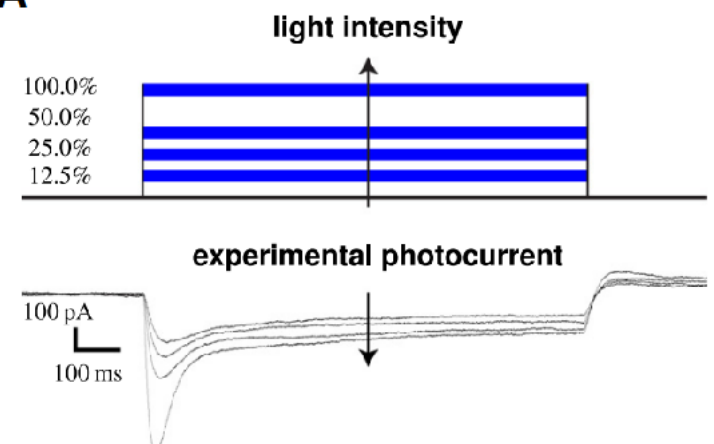

computational photocurrent

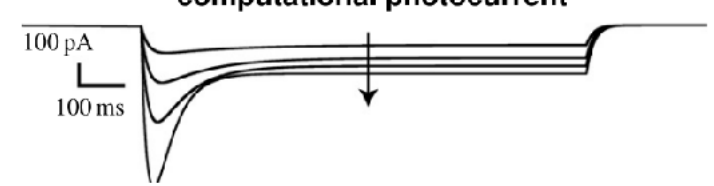

C

$$
\text { light stimulation at } 1.0 \mathrm{~Hz}
$$

Represented as: light stimulation, field potentials, mechanical contractions, computational models

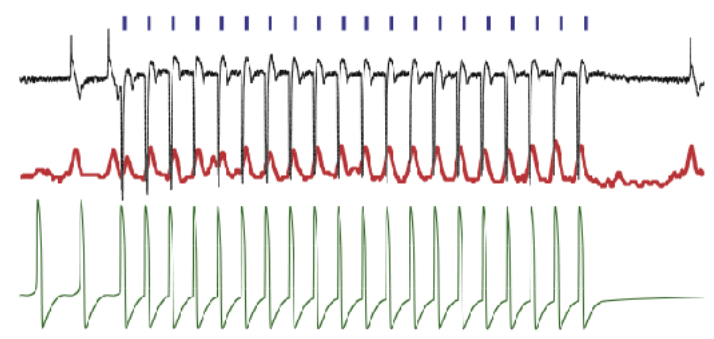

E

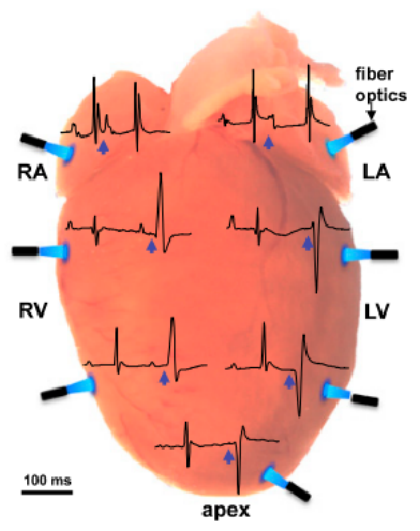

B

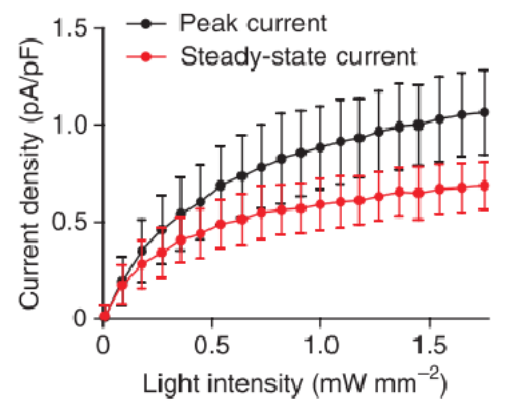

D

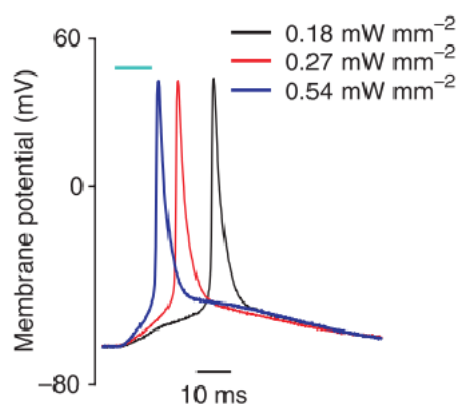

F

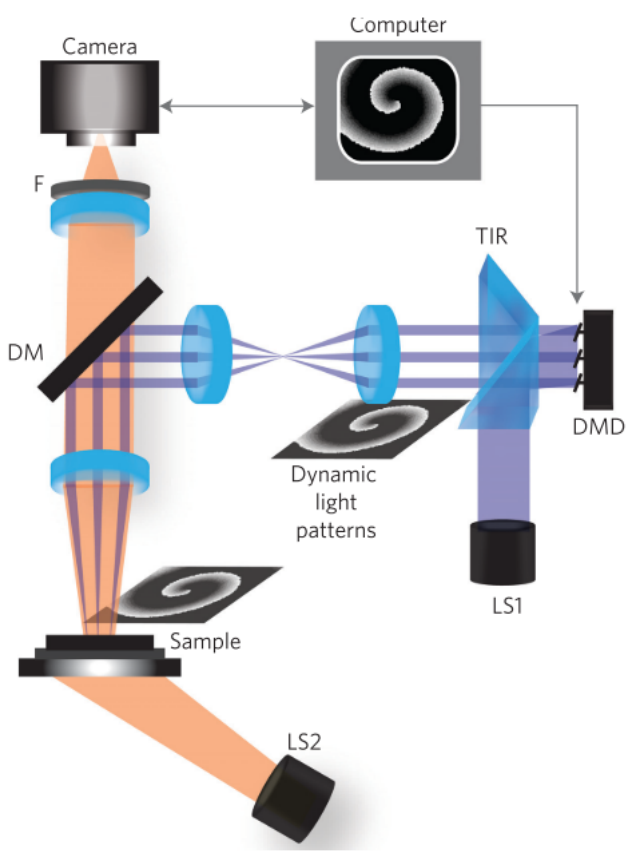

Figure 4.3.: Optogenetic control of cardiac electrophysiology. Caption on next page. 
Figure 4.3.: Optogenetic control of cardiac electrophysiology. Experimental and computational work has shown that the electrophysiological behaviour induced by ChR2 will depend on the intensity of the stimulus. (A) Photocurrents will be larger for higher intensities, both peak and steady-state currents (B). Additionally, (C) light stimulation of individual cells, cardiac monolayers or beating hearts will derive on temporal response and activation of the target, and on single cells (D) higher intensities will translate into faster triggering of action potentials. (E) Regional control was also proved using optical fibers to stimulate specific areas of the heart and confirmed on the ECG recordings. (F)Direction, speed and spiral chirality of excitation waves on cardiac monolayers was achieved using a micro-mirror device. Images obtained and modified with permission from [1] for A and C, [11] for B and D, [96] for E, [15] for F.

\subsection{Optogenetic arrhythmia termination}

One of the most ambitious and desired goals in the field of cardiac optogenetics is the possibility of pain-free and therefore harmless arrhythmia termination. A potential application could be seen in an optogenetic implantable cardioverter-defibrillator (ICD). Patients implanted with ICD not only suffer from pain at the moment of the shocks, but also from depression and anxiety showing a reduction in their self-perceived quality of life, mainly due to the application of irregular shocks and the development of other symptoms that affect the patients mentally and physically [79, 89].

The first proof of optogenetics-based arrhythmia termination was shown on monolayers of neonatal rat atrial cardiomyocytes [6]. Spiral waves were electrically induced and then terminated by photo-stimulation of the $\mathrm{Ca}^{2+}$-permeable channelrhodopsin CatCh [45], which is also sensitive to blue light. Light pulses of $500 \mathrm{~ms}$ at $0.038 \mathrm{~mW} / \mathrm{mm}^{2}$ led to arrhythmia termination in all the cell cultures where CatCh was expressed. Spiral termination was attributed to the decrease in excitability by the light-induced depolarization that lead to meandering and collision of phase singularities with one another or with the boundaries (Fig 4.4).

This study demonstrated the potential of light induced arrhythmia control. However, a translation to clinical applications is still many challenges away. Two of the most immediate ones are related to the translation from a 2D culture model to a whole-heart model. First, arrhythmia behaviour is more complex in a heart and the physical boundaries such as the ones found in a 2D model are not there. And second, light-penetration can become an issue. Bingen and colleagues relied on homogeneous illumination of the cardiomyocyte monolayers [6], therefore one can expect that almost all the cells received the same intensity. Achieving a stimulus as precise and as global as the one achieved by them is a practically impossible challenge, even without mentioning that simply achieving stimulation of all the cells in the heart could already be a challenge. 
A

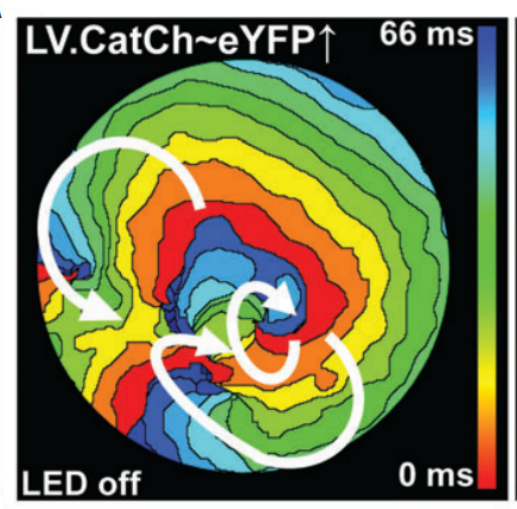

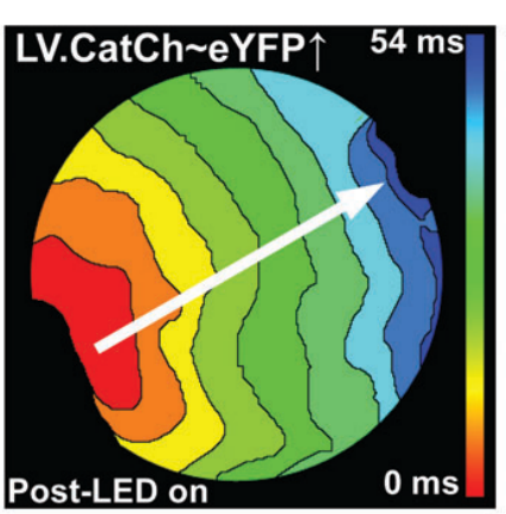

B

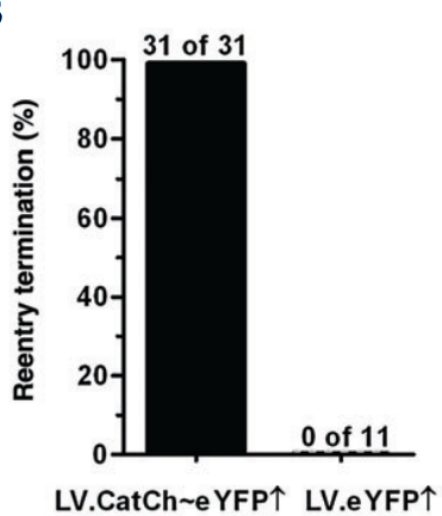

Figure 4.4.: Effective light-induced spiral wave termination by CatCh expression. (A) Activation map of CatCh-transduced rat atrial cultures showing spiral arrhythmic activity before being optogenetically stimulated (left) and after $500 \mathrm{~ms}$ of homogeneous illumination, showing planar wave eletrcial propagation. (B) Rate of successful reentry termination for CatCh-transduced cultures $(n=31)$ and cultures transduced only with eYFP $(n=11$, control). Image obtained and modified with permission from [6].

\subsubsection{First "optical shocks" in small animal models}

Nonetheless, in 2016 three different groups were able to revert arrhythmic hearts ex-vivo into their normal rhythm via optogenetical control of their electrophysiology. A combination of two different animal models, using two versions of channelrhodopsins and also diverse illumination strategies opened the road into a new dimension of cardiac arrhythmia research. While the works by Bruegmann and Crocini were done on ChR2-transgenic mice hearts [12, 19], Nyns and colleagues stimulated red-activatable channelrhodopsins (ReaChR) in rat hearts treated with the injection of adeno-associated virus [61].

Since the work of this thesis was performed using ChR2-transgenic mice hearts, the results obtained in the same model were actually used to set the foundations of the research performed and will be more broadly discussed. Using the $\mathrm{K}_{A T P}$ channel opener pinacidil, Bruegmann et al. were able to induce arrhythmia in the Langendorff-perfused mouse heart, and by illumination of the anteroseptal epicardium able to perform optogenetic cardioversion on the beating heart. $\mathrm{K}_{A T P}$ are potassium channels sensitive to adenosine triphophate (ATP) and have a protective role during ischaemia [28]. Their opening leads to the shortening of the action potential duration, facilitating the initiation of arrhythmias.

Compared to optogenetic pacing, cardioversion demands increased amounts of energy, which in optogenetics can be translated into into higher intensities, longer pulses and larger areas to illuminate. In this experiments it was also demonstrated that for the same reason length, intensity and area have an influence in the efficiency of the termination attempt (Fig 
A

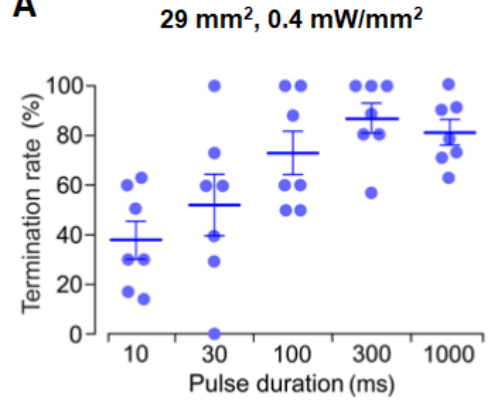

D

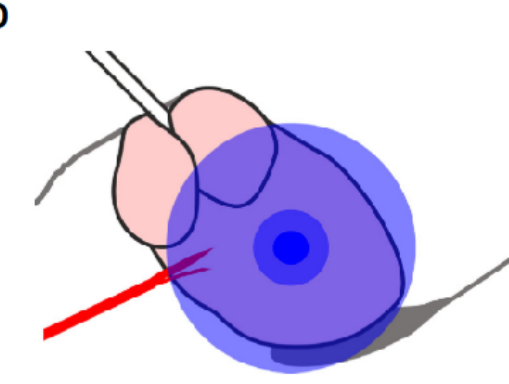

B

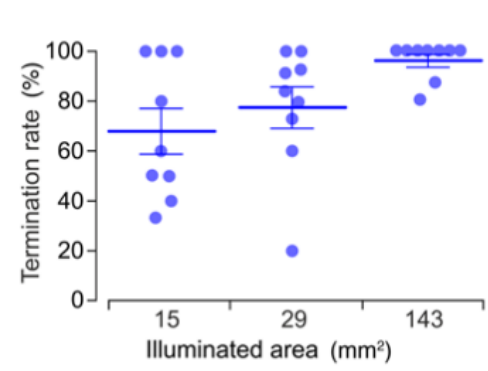

C

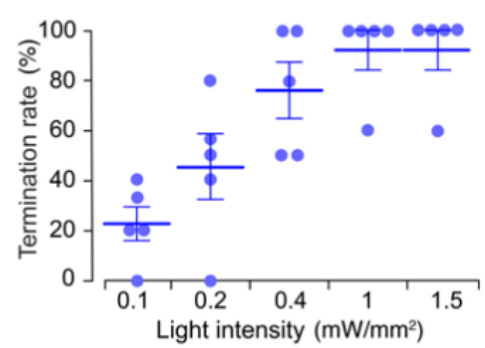

Figure 4.5.: Optogenetic defibrillation of the mouse heart by illumination of the epicardial surface. Stimulation of Langendorff-perfused hearts in arrhythmic state using a 4-pulse protocol shows that efficiency depends on (A) pulse length $(n=7)$, (B) area illuminated $(n=9)$ and $(\mathbf{C})$ the intensity delivered to the cardiac tissue $(n=5)$. For the parameters tested the pulse length maximum efficiency appears to be reached between 300 and $1000 \mathrm{~ms}$, while the intensity saturates at $1.0 \mathrm{~mW} / \mathrm{mm}^{2}$. (D) Arrhythmia was induced benefiting from the effects of pinacidil via electrical burst pacing and the stimulated protocol consisted of 4 light pulses of identical characteristics. Data presented as mean \pm S.E.M. Each data point represents the termination rate in one heart. Image obtained and modified with permission from [12]

4.5). In order to obtain a higher efficiency, the number of pulses was increased to 4, which allowed them to terminate at a rate of $97 \%$ with stimuli of $0.4 \mathrm{~mW} / \mathrm{mm}^{2}$ lasting 1 second on an area of $143 \mathrm{~mm}^{2}$. Additional experiments of clinical relevance performed by Bruegmann and colleagues in the same work demonstrated the feasibility of optogenetic cardioversion in hearts with induced acute myocardial infarction and in a different scenario the termination of arrhythmia wild type mice heart one year after gene transfer via AAV injection.

The approach of Crocini et al. was different. Even though they also looked to optogenetically terminate arrhythmia they aimed to take full advantage of the spatio-temporal benefits of 


\section{Cardiac Optogenetics}

using optogenetics. In order to do this they designed an imaging and stimulation setup using a macroscope and a laser scanning system based on acousto-optics deflectors. This allowed them to "draw" different illumination patterns on the surface of the heart (Fig 4.6).

A

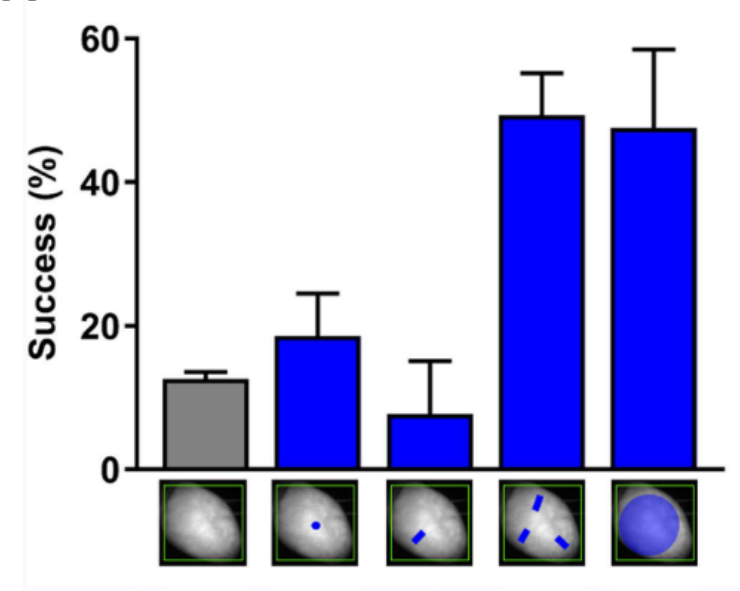

B

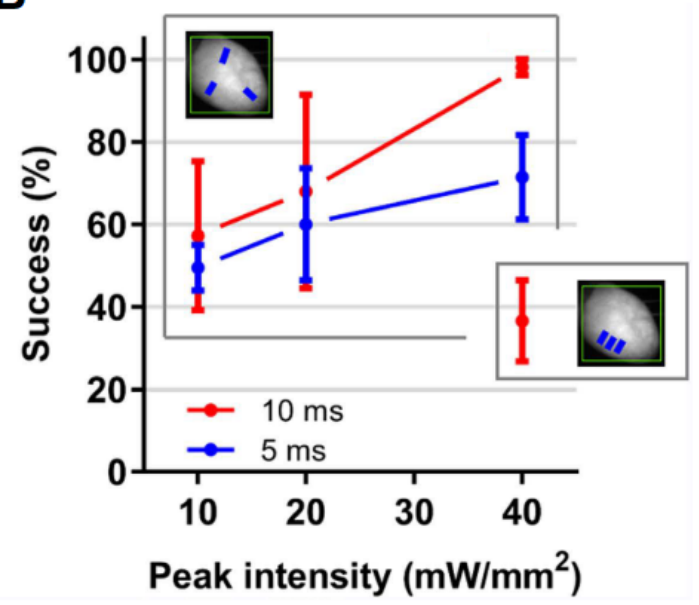

Figure 4.6.: Shaped illumination in optogenetic defibrillation. (A)Success rates for different shapes tested. The intensity of the triple barrier is $10 \mathrm{~mW} / \mathrm{mm}^{2}$ and whole $\mathrm{LV}$ was illuminated with an intensity of $0.5 \mathrm{~mW} / \mathrm{mm}^{2}$. Data presented as mean \pm S.E.M. (B)The triple barrier was tested using different intensities as well as two pulse durations. The highest success rate obtained using $40 \mathrm{~mW} / \mathrm{mm}^{2}$ and $10 \mathrm{~ms}$ was also compared to three barriers placed in a different position terminating only $36 \%$ of the attempts. Image obtained and modified with permission from [19].

Since the arrhythmias generated in their ChR2-mice hearts consisted of a re-entrant spiral covering the left ventricle they opted to use a triple barrier stimulation shape (Fig 4.6A) in order to depolarize the $\mathrm{ChRh} 2$-cardiomyocytes on this specific areas and generate conduction blocks along the path of the tachycardia.

With this concept, the triple barrier terminated arrhythmia at a rate of $98 \%$ covering a total area of $0.45 \mathrm{~mm}^{2}$ compared to Bruegmann's $143 \mathrm{~mm}^{2}$ using a series of 10 pulses of $10 \mathrm{~ms}$ at an intensity of $40 \mathrm{~mW} / \mathrm{mm}^{2}$, which is 100 -fold greater than the intensity used in the first approach.

As mentioned before, both groups used different manners in order to end arrhythmic behavior using optogenetics for the first time in the beating heart [20]. An advantage from the first group was the ability to terminate non-specific arrhythmia and do it at evidently lower intensities. On the other side it required 4 pulses of $1000 \mathrm{~ms}$ with "off" breaks of 1-5 seconds in between. Altogether this can be a great amount of time considering the physiology and heart rate of the mouse heart.

The second group, which needed only a total of $100 \mathrm{~ms}$ of stimulation and $1000 \mathrm{~ms}$ 
to complete the termination protocol suffered a compensation in energy by increasing the intensity up to $40 \mathrm{~mW} / \mathrm{mm}^{2}$, which would need validation of no harm induced on the cardiac tissue since phototoxicity is one of different challenges to be faced by cardiac optogenetics [69].

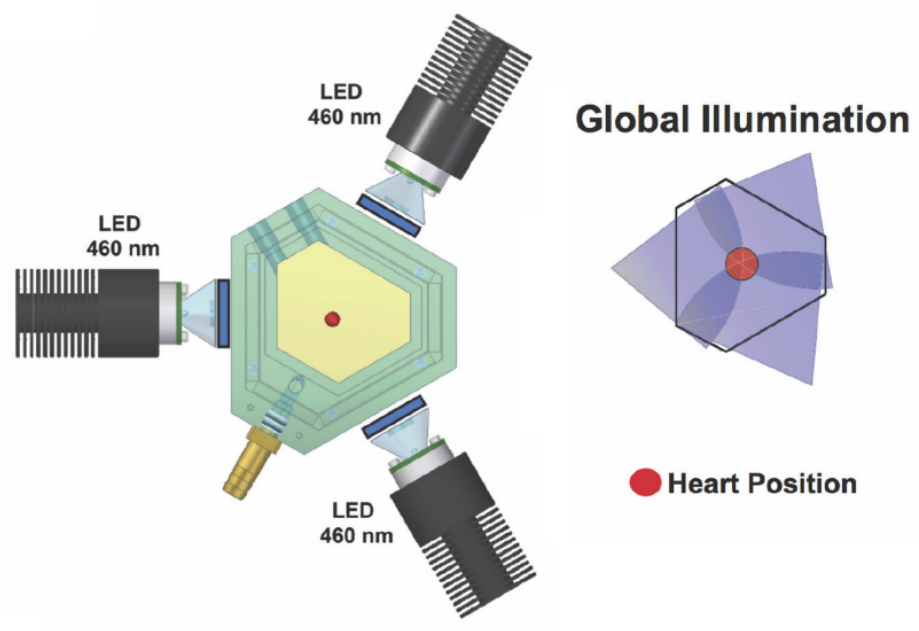

Figure 4.7.: Global illumination of the epicardial surface. A setup composed of three LEDs surrounding the heart enables simultanous illumination of the whole heart which could resemble electrical defibrillation. Image obtained and modified with permission from [67].

The most important part of this doctorate work was the aim to use yet a different approach that would tackle the disadvantages of the two methods previously described, which are the need of multiple and long stimulation pulses or the need of very high intensities. Therefore a stimulation setup illuminating the complete surface area of the heart was designed (Fig 4.7). Apart from allowing us to reduce the intensity needed and the length of the pulses, it would also help us understand how arrhythmia behave in a scenario similar to the application of electrical shocks, where the complete heart is stimulated. Chapter 7 is dedicated to the results and findings obtained with this aim.

\subsubsection{Mechanisms of optogenetic cardioversion}

Sasse and colleagues described optogenetic cardioversion via depolarization using ion channels as an outcome from two possible mechanisms; conduction block or filling of the excitable gaps [76]. An excitable gap is the area of the heart or tissue that has been depolarized and has had enough time to recover from the refractory period, and therefore is again excitable (Fig 4.8A).

During conduction block certain volume, thickness or area of the cardiac tissue is il- 
A

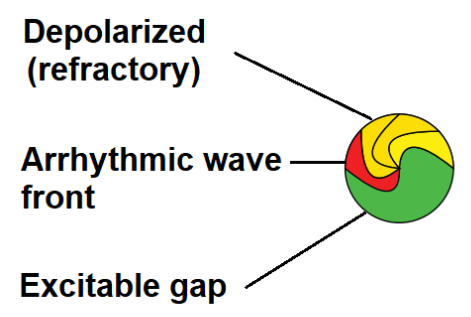

B

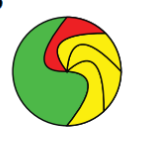

C

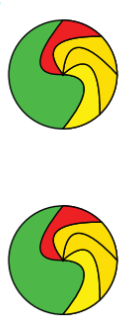

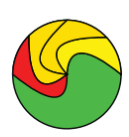
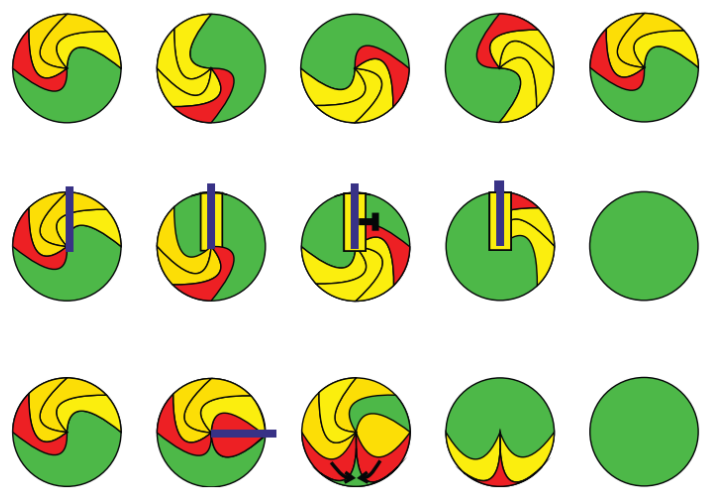

Figure 4.8.: Mechanisms of cardioversion by optogenetic depolarization. (A) After depolarization of the cardiac tissue occurs (red), it cannot be excited again for a period of time while it recovers (yellow), and lastly once it is recovered it is considered an excitable gap again (green). (B) Arrhythmic spiral wave at different time points, where the wave front travels and behind it the cardiac media shows refractorines for a period of time before becoming excitable again. (C) A conduction block consists of continuously illuminating an area of the tissue in order to keep it in a refractory period until the next arrhythmic wave front rout is blocked by the illuminated area. (D) Filling the excitable gap consists in illuminating part of the tissue that can be excitable in order to generate a new wave that would clash with the arrhythmic wave leading to its annihilation. Image obtained with permission from [76].

luminated and brought to an unexcitable state long enough to block or disrupt the activation path of the arrhythmia, therefore halting its activity (Fig $4.8 \mathrm{C}$ ). In this case, the amount of volume stimulated should be enough to properly create the block since a partial block can still allow circulation of the arrhythmic wave through non-excited or deeper areas of the ventricles [88, 41]. Filling of the excitable gap consists of stimulating an available region of the tissue in order to generate a depolarization wave that can collide with the arrhythmia and lead to the termination of both (Fig $4.8 \mathrm{D})$.

Creating a conduction block requires continuous stimulation of the arrhythmic wave path, hence illumination of the deeper layers of the heart will be of importance and can be a limiting factor. Differently, filling the excitable gap can be accomplished with a lower amount of light but it requires timing and positioning of the gap.

\subsubsection{Determinants in optogenetic cardioversion}

As previously mentioned, Nyns and colleagues accomplished optogenetic cardioversion on rat hearts using the red-activatable channelrhodopsins (ReaChR). More interestingly, they 
demonstrated first that the energy requirements for terminating monomorphic and polymorphic arrhythmia are also different. With a stimulation of a $1000 \mathrm{~ms}$-single pulse at an intensity of $2.97 \mathrm{~mW} / \mathrm{mm}^{2}$ on an area of $125 \mathrm{~mm}^{2}$ they achieved a success rate of $97 \%$ in the case of monomorphic tachycardia compared to only $57 \%$ when a polymorphic tachycardia was presented [61]. Furthermore, they suggested that stimulating the cardiomyocytes during the arrhythmia leads to an increase in their action potential duration (APD) which perturbs the arrhythmia facilitating termination.

In experiments performed on neonatal rat ventricular slices, Watanabe and colleagues exhibited the importance of penetration depth as a factor to finish arrhythmia using light (Fig 4.9] [88]. Re-entrant arrhythmia were triggered and conduction blocks of different thickness (transmurality) formed by light-induced depolarization were tested.

Their first step was to test whether termination was possible via global illumination of the slices. Once this was shown to be successful they moved into illuminating a specific width while still covering the transmurality or depth on its whole, therefore moving from global to local illumination. After finding an optimal thickness able to stop the re-entrant activity with the full depth they decided to further reduce the volume of the stimulus and make it even more local. They compared the efficiency of termination between 25, 50, 75 and 100\% depth, and the only stimulation volume capable reaching a success rate higher than $50 \%$ was the last one. This indicates that as long as the arrhythmic wave is left with excitable substrate to travel, the chances of termination will not increase dramatically.

There have also been different groups doing computational work that have helped in the understanding of the mechanisms and requirements of optogenetic defibrillation. Karathanos and colleagues simulated optogenetic termination of ventricular fibrillation (VF) in human heart models under different parameters [41]. In this model, 58\% of the cells expressed a lightsensitive ion channel and both the endocardial and epicardial surfaces were covered by grids of optrodes of $1 \mathrm{~mm}$ radius with consistent spacing. VF was induced and each termination attempt consisted in simultaneously turning on all the optrodes.

In order to investigate optimal parameters to achieve this goal, four different versions of channelrhodopsins were tested; ChR2-H134R (ChR2), which is the same used in the experiments of this doctoral work, a ChR2 version with increased sensitivity (ChR2+), and red activated variants of these two (ChR2-RED, ChR2-RED+). It is important to note that the last three are just modeled channels and there is no version of them available for experiments. Nevertheless, they were simulated in order to investigate the role of light penetration and attenuation of the cardiac tissue. Other parameters varied were the optrode density per area and the duration of the stimulus.

The results of these simulations exhibited that no arrangement of features where the blue-sensitive channelrhodopsins were used was successful at any optogenetic defibrillation attempt, confirming the importance of light penetration. A stimulation pulse of $500 \mathrm{~ms}$ using the highest density of optrodes, in a heart expressing the enhanced-sensitivity version of ChR2 

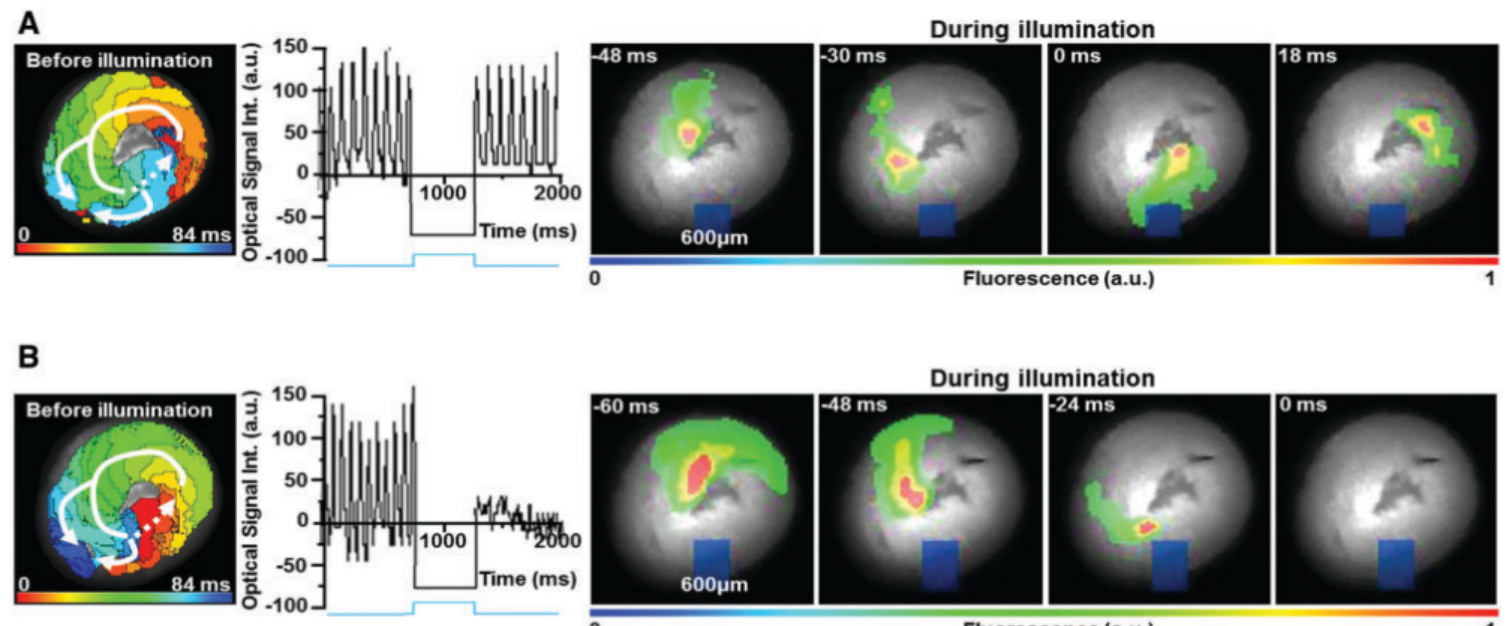

During illumination
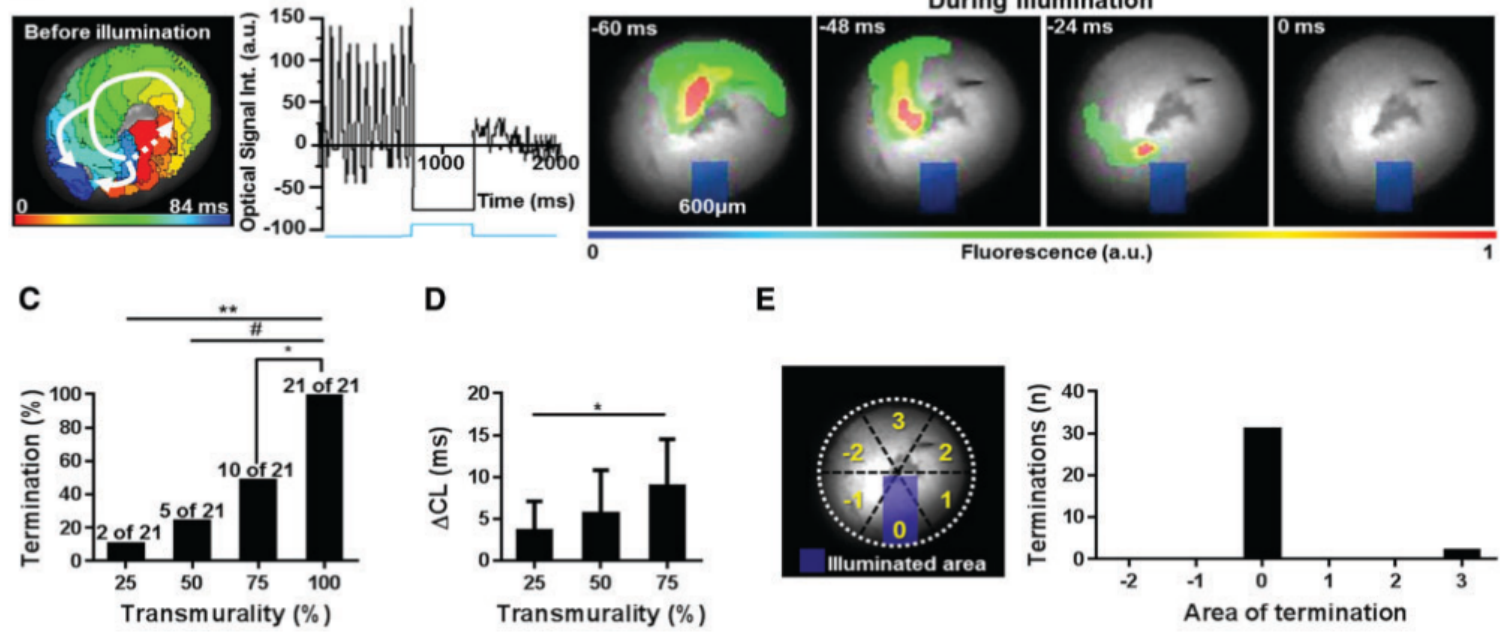

D

E
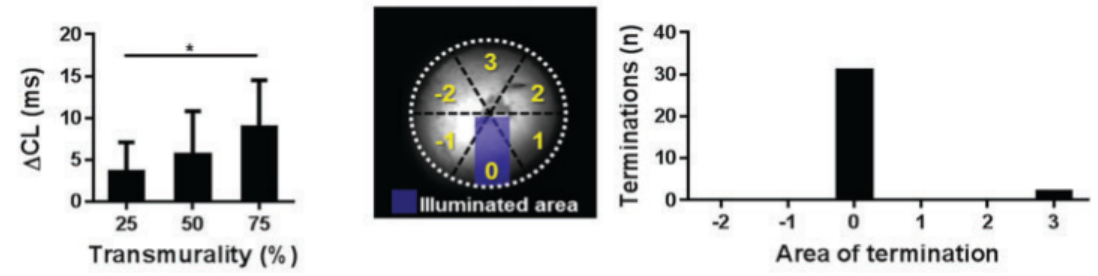

Figure 4.9.: Effect of illumination volume on the termination of re-entrant arrhythmias in CatCh ventricular slices. (A) Unsuccessful and (B) successful examples of cease in arrhythmic behaviour using different transmural illumination. On the left is shown an activation map before stimulation, followed by the optical signal obtained before and after stimulation and on the right the path followed by the electrical activity over time. (C) Effect of transmurality on the termination rate and (D) the cycle length of the re-entry.(E) All except for one arrhythmia were terminated in the illuminated area, validating termination due to optogenetic induced conduction block. Image obtained with permission from [88]

(ChR2+) activated only $10 \%$ of the ventricular tissue. Compared to $46 \%$ by solely substituting the ion channels' sensitivity from blue to red. Figure 4.10 illustrates the distribution of the transfection and optrode densities, and the table includes the ratio of ventricular activation based on different parameters such as the type of ion channel used, the pulse duration and the optrode density.

The enhancement of all these tested parameters translates into a higher volume of ventricular mass activated and the success rate mechanism is dependent on the stimulation of the excitable gap. The wavelength affects the penetration depth while the optrode density directly increases the amount of light. Moreover, a longer pulse will increase the chances of stimulat- 
A

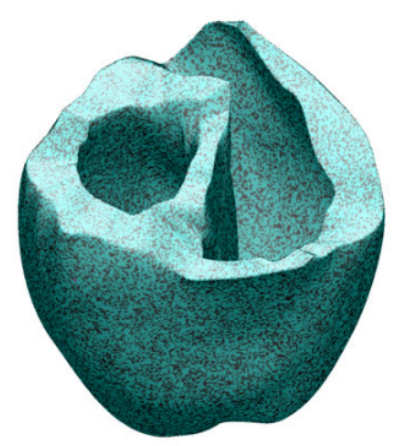

B

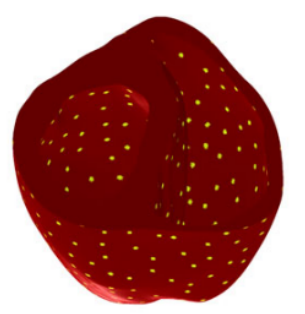

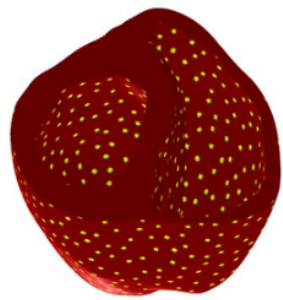

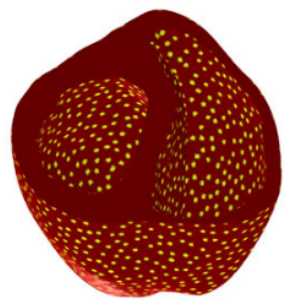

C

Percentages of light-activated ventricular tissue at three different optrode densities $\left(1.15,2.30 \mathrm{or} 4.61 \mathrm{~cm}^{-2}\right)$.

\begin{tabular}{lccccc}
\hline & Illumination & \multicolumn{3}{c}{ Optrode Density } \\
\cline { 3 - 5 } Opsin model & duration & $1.15 \mathrm{~cm}^{-2}$ & $2.30 \mathrm{~cm}^{-2}$ & $4.61 \mathrm{~cm}^{-2}$ & Calculated illumination threshold \\
\hline ChR2 & $25 \mathrm{~ms}$ & $1.37 \%$ & $2.76 \%$ & $5.54 \%$ & $0.08 \mathrm{~mW} \mathrm{~mm}^{-2}$ \\
ChR2 & $500 \mathrm{~ms}$ & $1.48 \%$ & $2.99 \%$ & $5.99 \%$ & $0.07 \mathrm{~mW} \mathrm{~mm}^{-2}$ \\
ChR2 & $25 \mathrm{~ms}$ & $2.08 \%$ & $4.18 \%$ & $8.47 \%$ & $0.04 \mathrm{~mW} \mathrm{~mm}^{-2}$ \\
ChR2+ & $500 \mathrm{~ms}$ & $2.45 \%$ & $4.94 \%$ & $10.0 \%$ & $0.03 \mathrm{~mW} \mathrm{~mm}^{-2}$ \\
ChR2-RED & $25 \mathrm{~ms}$ & $2.68 \%$ & $5.64 \%$ & $13.87 \%$ & $0.08 \mathrm{~mW} \mathrm{~mm}^{-2}$ \\
ChR2-RED & $500 \mathrm{~ms}$ & $3.05 \%$ & $6.47 \%$ & $16.6 \%$ & $0.07 \mathrm{~mW} \mathrm{~mm}^{-2}$ \\
ChR2-RED+ & $25 \mathrm{~ms}$ & $5.35 \%$ & $12.1 \%$ & $34.5 \%$ & $0.04 \mathrm{~mW} \mathrm{~mm}^{-2}$ \\
ChR2-RED + & $500 \mathrm{~ms}$ & $7.17 \%$ & $17.0 \%$ & $46.8 \%$ & $0.03 \mathrm{~mW} \mathrm{~mm}^{-2}$ \\
\hline
\end{tabular}

Percentages of light-activated ventricular tissue at three different optrode densities $\left(1.15,2.30\right.$ or $\left.4.61 \mathrm{~cm}^{-2}\right)$.

Figure 4.10.: Proportion of light-activated ventricular tissue. (A) Ventricular model displaying the distribution of the channelrhodopsins (blue) in the non-transfected tissue (black). (B) Examples of the different optrode densities modeled in human ventrical optogenetic cardioversion. $1.15 / \mathrm{cm}^{2}$ (left), $2.30 / \mathrm{cm}^{2}$ (middle), $4.61 / \mathrm{cm}^{2}$ (right). (C) Table indicating the proportion of tissue activated depending on the opsin, density and pulse length used. Image obtained and modified with permission from [41].

ing cells that with a short pulse would have been illuminated during the refractory period.

To summarize, there are different factors to be considered at the moment of optogenetically stimulating the heart, and all of these will play a different role in the task of optogenetic cardioversion.

As mentioned before, the aim of this doctoral thesis was to use global epicardial illumination of the heart to terminate arrhythmias and investigate the benefits of global illumination as an alternative to structured or local illumination. Allowing us to apply smaller intensities and to better understand the behavior of arrhythmia under optogenetic arrhythmia termination. Moreover, global illumination can offer a closer comparison to electrical cardioversion where the whole heart is electrically stimulated. The next chapter is focused on describing the methods used before moving into Part $\Pi$, which contains the chapters reporting the results 
4. Cardiac Optogenetics

obtained. 


\section{Materials \& Methods}

\subsection{Light transmittance of the cardiac tissue}

The transmittance of light by the heart and different cardiac structures (LV, RV and septum) was estimated using a setup that consisted of a LED illuminating the epicardial surface and a S120VC photodiode power sensor connected to the PM100D optical power meter (Thorlabs) to detect the light coming through the tissue across a pinhole of $1 \mathrm{~mm}$ in diameter (Figure 6.2). The transmittamce of light was obtained from the ratio of the light coming through the tissue and the light directly from the LED through the pinhole without any cardiac tissue. Two LEDS of different wavelengths were implemented. One with a wavelength of $625 \mathrm{~nm}$ (M625L3, Thorlabs) with a bandpass filter (FF02-628/40-25, Semrock) and the other with a wavelength of $460 \mathrm{~nm}$ (M470L3) with a bandpass filter 470(20) nm (ET470/40x, Chroma). The transmittance of 5 different intensities in the range between $0.5 \mathrm{~mW} / \mathrm{mm}^{2}$ to $1.5 \mathrm{~mW} / \mathrm{mm}^{2}$ was considered to calculate the mean values of each heart. Light transmittance was measured in hearts under different conditions; either a) with blood (directly after extraction of the heart from the mouse), b) after 10 min of Langendorff-perfusion with Tyrode, c) perfusion with Tyrode for $10 \mathrm{~min}$ plus $20 \mathrm{~min}$ of perfusion with $5 \mu \mathrm{M}$ Blebbistatin/Tyrode, or d) 10 min Tyrode perfusion followed by a bolus injection of $50 \mu \mathrm{M}$ of the voltage dye Di-4ANBDQPQ (Thermo Fisher Scientific) and perfusing for another 5 minutes. Due to the nature of this experiment, not more than one condition could be tested in the same heart. Transgenic ChR2 mice hearts with Blood $(n=6)$, with Tyrode $(n=6)$, with Dye $(n=3)$ and with Blebbistatin $(n=4)$. Wild type mice hearts with Blood $(n=3)$ and with Tyrode $(n=3)$.

\subsection{Langendorff perfusion}

$\alpha$-MHC-ChR2 transgenic mice were heparinized and anesthetized using isoflurane before being sacrificed for heart explantation. Light absorption as well as pacing measurements were performed by Langendorff-perfusing the hearts with a Tyrode solution consisting of

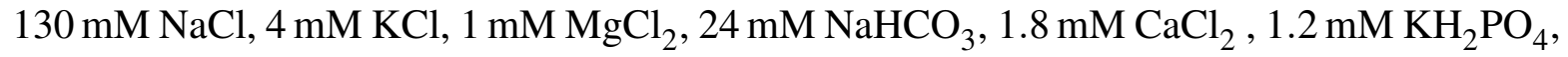
$5.6 \mathrm{mM}$ glucose, $1 \%$ albumin/BSA were aerated with carbogen $\left(95 \%\right.$ oxygen and $\left.5 \% \mathrm{CO}_{2}\right)$ as previously described in [70]. For arrhythmia induction, the solution was modified as detailed later in this chapter. The electrical heart activity was recorded using a monophasic action 
potential (MAP) electrode (BIOPAC Systems, Inc.).

\subsection{Investigation of pacing thresholds using optical fibers}

The minimum intensity required to pace the heart at each of the ventricles was tested by positioning the tip of an optical fiber of $\mathrm{d}=400 \mu \mathrm{m}$ connected to a LED of $460 \mathrm{~nm}$ in contact with the center of the right/left ventricle. Two different pulse widths were applied ( $5 \mathrm{~ms}$ and $10 \mathrm{~ms}$ ), and the intensity was step-wised decreased until the minimum intensity that initiated a response with a capture of $1: 1$ of the last 10 pulses from a train of 20 pulses for $n=5$ attempts in a row for each heart was found. Threshold intensities were examined for the RV and LV under normal Tyrode and after the injection of the membrane potential dye. The pacing threshold of hearts perfused under normal Tyrode vs. the effect of $5 \mathrm{mM}$ Blebbistatin/Tyrode after 20 min of perfusion was also examined on the LV. $N=6$ control hearts and hearts with blebbistatin, $N=3$ hearts with dye.

\subsection{Pacing with different intensities, pulse lengths and surface areas}

To investigate the response rate of the heart to different kinds of stimuli, four different light intensities were chosen for each optical fiber based on previous results. The intensity units used throughout the thesis are milliwatt per millimeter square $\left(\mathrm{mW} / \mathrm{mm}^{2}\right)$. For the $\mathrm{d}=400 \mu \mathrm{m}\left(\mathrm{a}=0.126 \mu \mathrm{m}^{2}\right)$, the intensities investigated were $1.8 \mathrm{~mW} / \mathrm{mm}^{2}, 2.3 \mathrm{~mW} / \mathrm{mm}^{2}$, $3.5 \mathrm{~mW} / \mathrm{mm}^{2}$ and $5 \mathrm{~mW} / \mathrm{mm}^{2}$ and for the $\mathrm{d}=1000 \mu \mathrm{m}\left(\mathrm{a}=0.785 \mu \mathrm{m}^{2}\right)$ the intensities were lower, $0.4 \mathrm{~mW} / \mathrm{mm}^{2}, 0.7 \mathrm{~mW} / \mathrm{mm}^{2}, 1 \mathrm{~mW} / \mathrm{mm}^{2}$ and $2.2 \mathrm{~mW} / \mathrm{mm}^{2}$. For each fiber and intensity, the heart was illuminated using a train of 20 light pulses and the last 10 were considered to determine the capture rate of that heart for that combination. In order, pulses of $1 \mathrm{~ms}$, $2 \mathrm{~ms}, 3 \mathrm{~ms}, 4 \mathrm{~ms}, 4 \mathrm{~ms}, 5 \mathrm{~ms}, 6 \mathrm{~ms}, 7 \mathrm{~ms}, 8 \mathrm{~ms}, 9 \mathrm{~ms}, 10 \mathrm{~ms}$ and $15 \mathrm{~ms}$ were tested. $N=5$ hearts were assayed for $\mathrm{d}=400 \mu \mathrm{m}$ and $N=4$ for $\mathrm{d}=1000 \mu \mathrm{m}$. 
The following methods were first used in "Energy-Reduced Arrhythmia Termination Using Global Photostimulation in Optogenetic Murine Hearts" [67] (Chapter 7) and they were also used in Chapters 8,9 therefore they are described here again as described in [67].

\subsection{Arrhythmia Induction [67]}

In order to induce sustained arrhythmia we lowered the concentration of $\mathrm{KCl}$ to $2 \mathrm{mM}$, so that the arrhythmia induction tyrode contains $130 \mathrm{mM} \mathrm{NaCl}, 4 \mathrm{mM} \mathrm{KCl}, 1 \mathrm{mM} \mathrm{MgCl}{ }_{2}$, $24 \mathrm{mM} \mathrm{NaHCO}_{3}, 1.8 \mathrm{mM} \mathrm{CaCl}_{2}, 1.2 \mathrm{mM} \mathrm{KH}_{2} \mathrm{PO}_{4}, 5.6 \mathrm{mM}$ glucose, $1 \%$ albumin/BSA were aerated with carbogen (95\% oxygen and $5 \% \mathrm{CO}_{2}$ ). Because of a reduction in transmural dispersion of repolarization the so induced hypokalemia enhances arrhythmia induction [43]. In addition, $100 \mu \mathrm{M}$ Pinacidil, which is a $K_{A T P}$ channel activator, was applied to shorten the action potential duration (APD) [30, 91] . The combination of both factors has been successfully applied to induce long sustained ventricular arrhythmia in murine Langendorff-perfused hearts [12]. Sustained arrhythmia was induced by applying 30 electrical pulses with a needle electrode in the range of $30 \mathrm{~Hz}$ to $50 \mathrm{~Hz}$. All perfusion experiments were done at $37^{\circ} \mathrm{C}$.

\subsection{Global Illumination for Cardioversion [67]}

Global illumination was applied in all the optogenetic cardioversion experiments. In order to achieve a consistent optogenetic stimulation of the whole heart surface and therewith global illumination, the hearts were vertically arranged surrounded by three blue-light emitting diodes (blue-LED, Thorlabs) with their wavelengths centered at $460 \mathrm{~nm}$ and limited by a 470(20) nm bandpass filter (ET470/40x, Chroma)(Figure 7.1). Synchronous millisecond control of LED at different intensities was conducted via a function generator (Arbitrary Function Generator A2230, Agilent Instruments). Intensity measurements were done using the PM100D optical power meter and the S120VC photodiode power sensor (Thorlabs). Since the experimental setup consists of three blue-LED spaced at $120^{\circ}$, the intensity was measured directly facing each LED from the heart position and the calculated mean was considered the overall light intensity during global illumination.

To determine the success rate of arrhythmia termination, we induced multiple arrhythmia in each heart and attempted termination 10 times with each light intensity-duration combination. To consider an arrhythmia as sustained we waited for $5 \mathrm{~s}$ after induction before attempting termination, which consisted of illuminating the whole heart by simultaneously turning on the three LED for the duration and intensity tested. If the arrhythmia stopped within maximum one second after the conclusion of stimulation, it was considered a successful attempt. In case of failed termination, we applied backup defibrillation, which consisted on 
increasing the intensity and duration of the pulses. To support the defibrillation procedure the hearts were perfused with maintenance tyrode. The parameter combination with the highest termination rate and the lowest pulse duration values were determined as the most efficient ones. They also served as a decision-critical point for parameter change.

\subsection{Optical Mapping [67]}

To diminish motion artifacts in optical records, the contraction uncoupling reagent Blebbistatin ( $c=5 \mu \mathrm{M}$, Thermo Fisher Scientific) was administrated. Potientiometric staining was achieved by the red-shifted dye Di-4-ANBDQPQ $(c=50 \mu \mathrm{M}$, Thermo Fisher Scientific) via bolus injection. A longpass $685 \mathrm{~nm}$ dichroic mirror (FF685-Di02-25x36, Semrock) was integrated to reflect the excitation light from a $625 \mathrm{~nm}$ mounted LED (M625L3, Thorlabs) after a bandpass filter (FF02-628/40-25, Semrock) onto the heart. The emission light was collected with a 775(70) nm bandpass filter (FF01-775/140-25, Semrock) before reaching the camera (see Figure 7.1). Epicardial signal recording was done with an electron multiplying charged coupled device (EMCCD, Cascade 128+, Photometrics) camera with a spatial resolution of 64 pixels x 64 pixels at $1 \mathrm{kHz}$. Camera control was achieved using custom-made recording software and the electrical heart activity was recorded using a monophasic action potential (MAP) electrode (BIOPAC Systems, Inc.).

The obtained fluorescent images were analyzed and processed using MatLab (MathWork, Inc.). Briefly, spatial and temporal smoothing filter were applied after pixelwise normalization. Overlapping of the blue light with the emission signal of the dye was removed by subtracting the average difference in intensities with and without blue light from each pixel over time. In the cases of short pulses $(2 \mathrm{~ms}, 5 \mathrm{~ms}, 5 \mathrm{~ms}$ and $10 \mathrm{~ms})$, where it was not possible to obtain an average of the signal during illumination, the illumination frames were completely removed and replaced by frames with no signal. The estimation of the total surface area of both ventricles and atria was achieved by reconstructing three-dimensional heart shapes from photographs using a shape from contour approach as previously described [18]. In total three hearts were used to calculate an average epicardiac surface area of $274 \mathrm{~mm}^{2}$.

\subsection{Statistical tests}

Statistical analysis was done applying the Student's t-test, two-sided and unpaired. A detailed description of the method is included in the following subsection. 


\subsubsection{Unpaired t-tests}

t-tests are statistical tests used to compare the means of two populations. Also known as Student's t-tests, these parametric tests are used to determine if there is a significant difference between the mean of two samples. The t-test only works for two samples and it helps find a difference that is unlikely to be due to sampling error or random chance [87, 72].

A paired t-test compares means of two matched or related groups, while an unpaired or independent t-test compares the means of two unmatched or unrelated groups to determine if there is a statistically significant difference. Both tests assume that the data was collected from populations following a Gaussian distribution. In an unpaired t-test it is also assumed that both populations have the same variance. There are two hypothesis in a t-test: The null hypothesis states the two means tested are equal, that the difference between them is zero $\left(\mathrm{H}_{0}: \bar{x}=\bar{y}\right)$. The alternative hypothesis states that there is a statistically significant difference between the two means tested $\left(\mathrm{H}_{1}: \bar{x} \neq \bar{y}\right)$. This is also known as a two-tailed t-test since either $\bar{x}$ or $\bar{y}$ can be the largest one. Ina one-tailed t test, we have to specify which mean we expect to be the larger one.

Whenever we perform statistical analysis, we are testing the some hypothesis, this case the null hypothesis $\left(\mathrm{H}_{0}: \bar{x}=\bar{y}\right)$. The outcome of the statistical test will allow us to accept or reject the hypothesis and do so with some level of confidence. As mentioned before, in order to perform this statistical test we are assuming that the distributions of both populations are normal and that the variances are the same (or close to). Using the sampled data from the populations, the test statistic is calculated as:

$$
t=\frac{\bar{x}-\bar{y}}{\sqrt{s_{p}^{2}\left(\frac{1}{n_{1}}+\frac{1}{n_{2}}\right)}}
$$

where $S_{p}$ is the pooled standard deviation of the two samples:

$$
s_{p}^{2}=\frac{\left(n_{1}-1\right) S_{x}^{2}-\left(n_{2}-1\right) S_{y}^{2}}{n_{1}+n_{2}-2}
$$

$n_{1}$ is the number of observations for population $x$ and $n_{2}$ for population $y$, and $S_{x}^{2}, S_{y}^{2}$ their respective variances, $\bar{x}$ the sample average of $x$ and $\bar{y}$ the sample variance of $y$.

After the $t$ statistic has been obtained, the t-value is compared to t-values given in a table for the $\mathrm{t}$-distribution. Our value must be greater than the t-value entry to reject the null hypothesis with a (1- $\alpha)$ level of confidence for a one-sided t-test or (1- $\alpha / 2)$ for a two-sided 
t-test, which is this specific case. $\alpha$ is the level of significance at which we want to accept or reject our hypothesis. Values of $\alpha \leq 0.05$ are commonly used and a standard accepted to reject the null hypothesis. In other words, a confidence level of $95 \%$ or higher. Therefore, if we want to reject our null hypothesis $\left(\mathrm{H}_{0}\right)$ with a confidence level of $95 \%$, for a two-sided t-test our obtained t-value must be greater than $t\left(0.05 / 2, n_{1}+n_{2}-2\right)$. If this condition is met, we can reject $\mathrm{H}_{0}$ and accept $\mathrm{H}_{1}$ with a $95 \%$ confidence. $n_{1}+n_{2}-2$ is also known as the degrees of freedom $d f$.

\subsection{Determination of outliers for cardioversion times}

Outliers were determined using two different methods. The first method consisted of identifying the median and discarding from the samples the $10 \%$ that had the greatest distance from it, either larger or smaller. As comparison we also used the ROUT method [56] that combines robust regression with outlier detection. First it fits a curve using a robust nonlinear regression method that gives very little weight to extreme outliers, then by analyzing the residuals of the robust fit the outliers are determined by applying a test adapted from the False Discovery Rate approach. And lastly the outliers are removed and an ordinary least-squares regression is performed on the remaining data. A detailed description of the method can be found in [56]. 


\section{Part II.}

\section{Results}





\section{Optogenetic Characterization of the ChR2 Mouse Heart}
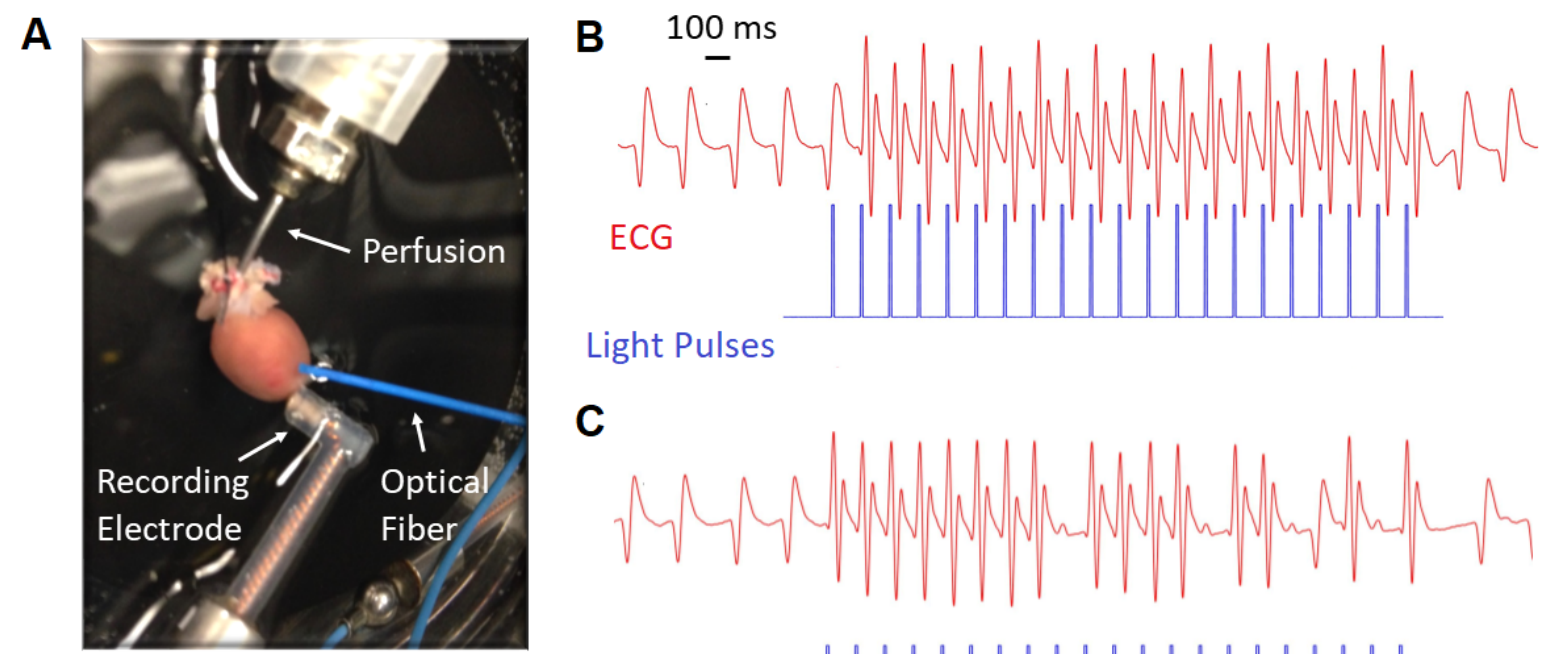

Light Pulses

C

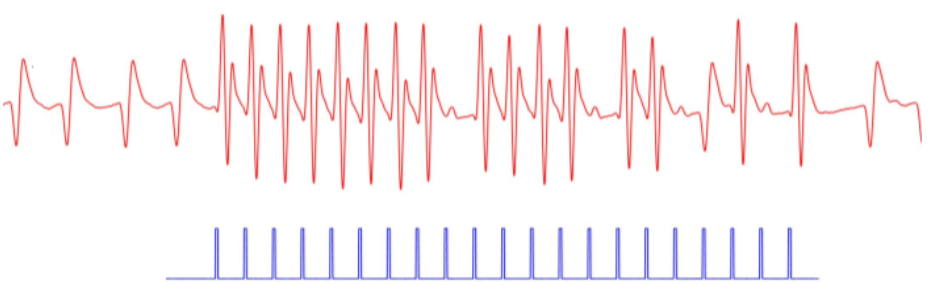

Figure 6.1.: Optogenetic pacing of the ChR2 Mouse Heart. (A) The heart is connected to the perfusion system and an optical fiber is employed to apply $470 \mathrm{~nm}$ light pulses at different widths and intensities while the electrical activity of the heart is being recorded using a monophasic action potential electrode. The recording can be seen on the right side; (B) shows 1:1 response of the heart to the last 10 pulses of the train of 20 light stimuli. (C) represents a different train of light pulses of lower intensity resulting in pulses that did not reach the pacing threshold to ignite a response in the Channelrhodopsin-2 heart.

\subsection{Introduction and Aim}

The energy necessary to stimulate a ChR2-transgenic heart depends on biological and physical aspects such as the electrophysiological properties of the cell expressing the channel, expression density and distribution of the opsin, the region of the heart stimulated, the intensity of the light used as well as the surface area illuminated. The aim of this thesis 
was to apply optogenetics-based cardioversion on the myocardium, therefore both light intensity and duration play a central role. Several other groups have investigated how these parameters acting on photo-sensitized cardiac tissue. The first experiments performed on single cardiomyocytes showed that longer pulses require lower intensities to reach a 1:1 capture (stimulation:response) rate, that higher intensities induce greater currents and decreases the time it takes action potential to reach its peak [11]. In the same work, Bruegmann et al. demonstrated that in vivo atrial stimulation of ChR2-expressing mice hearts demanded more light than the ventricles and that smaller areas required higher intensities to obtain a similar response. This could be related to the volume of cardiac tissue stimulated; a smaller area with a higher intensity would mean that the light is penetrating deeper and could compensate the total volume of tissue excited. Also in transgenic murine hearts, Zaglia et al. showed the differences in excitation thresholds between right (lower) and left ventricles for different areas and intensities [96]. Other results in stimulation thresholds have been shown for different shapes and areas [19], expression levels [85], as well as in rat models [60].

Light behaves differently under varying conditions, but in a simple scenario light it is either absorbed or not. What happens and to which proportion will depend in general on two factors: the wavelength of the photons emitted and the optical properties of the material (in this case the different types of cardiac tissue). When the light is not absorbed it can be scattered by the tissue. When the light is absorbed it can lead to many different events such as triggering chemical reactions (like in the case of Channelrhodopsin-2), it can also be transformed into heat (which could be harmful for cells), and light could also lose energy and change its wavelength through fluorescence (being the case of voltage sensitive dye) [86, 36].

In order to estimate and model light attenuation through the cardiac tissue, different groups have used the Lambert-Beer law [96, 9]:

$$
I(x)=I_{0} e^{-\mu x}
$$

where $I_{0}$ is the light intensity at the surface of the cardiac tissue (depth $\mathrm{x}=0 \mathrm{~mm}$ ), $\mu$ is the attenuation coefficient which is defined by the probability of a photon being absorbed in a specific material per unit path length and it is wavelength dependent, $x$ is the depth of the intensity estimation and $I(x)$ is the intensity at that depth. The ratio $I(x) / I_{0}$ is defined as transmittance $T(x)$ :

$$
T(x)=I(x) / I_{0}=e^{-\mu x}
$$

Therefore, the law of Lambert-Beer can be used to estimate the intensities at different depths into the cardiac tissue as well as the depth reached by blue or red light before a decline in intensity to levels where an optogenetic stimulus can not be produced. 
Blebbistatin is an electro-mechanical uncoupler used to stop the beating of the heart while maintaining its electrical activity and it is used in experiments in order to perform optical mapping. However, studies have shown that the effect of blebbistatin is inhibited via blue light [74] and that it also exhibits fluorescence upon excitation at $450 \mathrm{~nm}$. Di-4-ANBDQPQ is a voltage-sensitive dye used in optical mapping. It has its absorption peak at $603 \mathrm{~nm}$, however it also has a small peak of absorbance around $450 \mathrm{~nm}$ [54]. And lastly, blood absorbs and scatters light at different proportions depending on the wavelength and while its absorption coefficient for wavelengths $\sim 450 \mathrm{~nm}$ is close to $2 \mathrm{~mm}^{-1}$, its value decreases approximately 10 times in the case of wavelengths $\sim 650 \mathrm{~nm}[71]$.

Optimal optogenetic stimulation and arrhythmia termination will depend on these and other different factors that will affect how light travels in the cardiac tissue and how much light is delivered to the light-sensitive channels throughout cardiac walls [9, 41, 12]. Ex vivo experiments using the Langendorff technique [5] are the main source of research of this doctorate work and the conditions in which the heart will be optogenetically stimulated will differ from experiment to experiment. Moreover, the main subject of my study is the $\alpha$-MHC-ChR2 transgenic mouse line and these hearts might show different optical properties compared to wild type mice hearts.

Considering all the above mentioned factors, it was necessary to optogenetically characterize the ChR2 transgenic mouse heart. In other words, to define how this specific heart model responds to optogenetic stimuli with different paramenters (pulse length, intensity and area illuminated) and how the addition of substances such as blebbistatin or Di-4-ANBDQPQ affect the stimulation thresholds compared to a tyrode-only perfusion.

\subsection{Results}

\subsubsection{Light Transmittance by the Murine Cardiac Tissue}

Before stimulating the heart under different conditions, I aimed to interrogate light transmittance through the heart under different conditions. The goal of this experiment was to improve knowledge and understand if a change in the experiment (like adding dye or blebbistatin) can potentially affect stimulation thresholds and how do they affect in general on any given mouse heart prior to or during the experiment. Therefore, we decided to measure the mean transmittance of the different mice hearts, ventricles and septum in general without considering the specific thickness for each one of them, especially considering they were all healthy mice and no abnormally enlarged or shrunken hearts were expected. This would give us a head start on where and under which conditions the stimulation thresholds might increase or decrease. 
In order to estimate the light transmittance by the heart, more specifically by the ventricles and septum, we designed the experimental setup shown in Figure 6.2. The sample tissue was illuminated and the light intensity transmitted through the tissue was measured using a photometer through a $1 \mathrm{~mm}$ in diameter pinhole. Detailed methods are explained in section 5.2 Materials \& Methods.

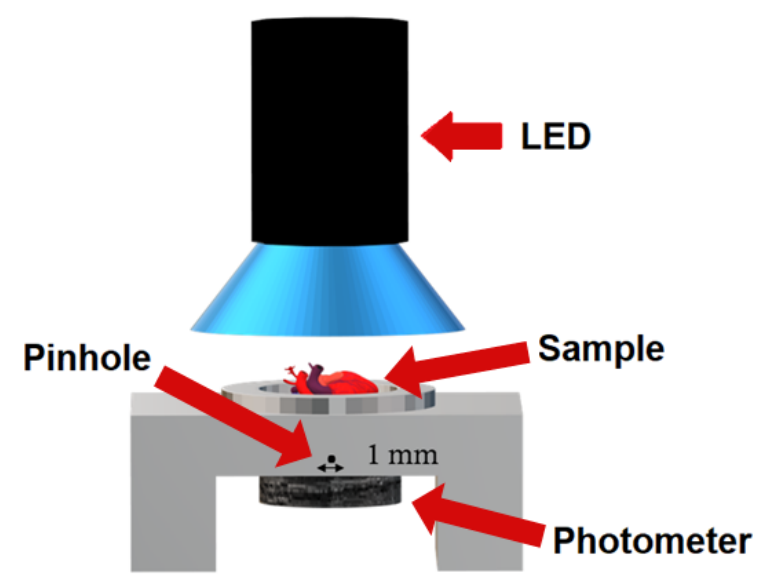

Figure 6.2.: Setup for light attenuation measurements. Light is emitted from a LED, coming down through the tissue and detected on the other side of a $1 \mathrm{~mm}$ in diameter pinhole. Used with permission from [2].

The differences in attenuation by the cardiac tissue were measured directly after one of the following conditions:

- perfusion with tyrode,

- perfusion with tyrode and injected with the potentiometric dye Di-4-ANBDQPQ, with a broad excitation spectra with its peak at $603 \mathrm{~nm}$ [54],

- perfusion with tyrode and the electromechanical uncoupler Blebbistatin,

- with blood, right after extraction.

In addition, two different wavelengths were used; $470 \mathrm{~nm}$ which is used to stimulate $\mathrm{ChR} 2$, and $625 \mathrm{~nm}$, we used to excite the potentiometric dye during optical mapping and can also be used in the future to stimulate other opsins. Table 6.1 summarizes the results obtained shown as percentage of transmitted light (intensity with sample/intensity without sample).

As expected, the RV transmitted more light than the LV, ranging from 5-10 times depending on the condition. When comparing the transmittance of light at different wavelengths, $470 \mathrm{~nm}$ transmittance was at least 5 times smaller for all the cases than $625 \mathrm{~nm}$, while in the LV and RV for the Tyrode perfused case in the ChR2 heart the difference was about 14-fold 


\begin{tabular}{|c|c|c|c|c|c|c|}
\hline & & & & ligher light & ttenuation & \\
\hline $\begin{array}{l}\text { perc } \\
\text { tras }\end{array}$ & $\begin{array}{l}\text { ntage } \\
\text { tted li }\end{array}$ & t [\%] & Tyrode & Dye & Blebbistatin & Blood \\
\hline & $\mathrm{ChR} 2$ & 470 & $0.21 \pm 0.09$ & $0.14 \pm 0.07$ & $0.07 \pm 0.04$ & $0.03 \pm 0.01$ \\
\hline Whole & WT & $4 / 0 \mathrm{~nm}$ & $0.22 \pm 0.03$ & & & $0.08 \pm 0.01$ \\
\hline heart & $\mathrm{ChR} 2$ & 620 & $1.8 \pm 0.3$ & $1.1 \pm 0.2$ & & $0.52 \pm 0.17$ \\
\hline & WT & $030 \mathrm{~nm}$ & $1.9 \pm 0.5$ & & & $0.5 \pm 0.1$ \\
\hline & $\mathrm{ChR} 2$ & 170 n & $0.27 \pm 0.07$ & $0.21 \pm 0.06$ & $0.17 \pm 0.04$ & $0.12 \pm 0.05$ \\
\hline Left & WT & $470 \mathrm{~nm}$ & $0.6 \pm 0.2$ & & & $0.22 \pm 0.09$ \\
\hline Ventricle & $\mathrm{ChR} 2$ & 620 n & $3.8 \pm 0.7$ & $2.3 \pm 0.4$ & & $1.9 \pm 0.3$ \\
\hline & WT & $630 \mathrm{~nm}$ & $3.8 \pm 1.3$ & & & $2.0 \pm 0.7$ \\
\hline & $\mathrm{ChR} 2$ & 170 n & $1.0 \pm 0.3$ & $0.6 \pm 0.1$ & $0.4 \pm 0.2$ & $0.3 \pm 0.01$ \\
\hline$S_{1}$ & WT & $470 \mathrm{~nm}$ & $2.2 \pm 1.2$ & & & $0.8 \pm 0.4$ \\
\hline Septum & $\mathrm{ChR} 2$ & 620 & $8.5 \pm 2$ & $4.1+0.6$ & & $4.0 \pm 0.9$ \\
\hline & WT & $030 \mathrm{~nm}$ & $8.6 \pm 1.5$ & & & $5 \pm 1.9$ \\
\hline & $\mathrm{ChR} 2$ & 470 m & $2.1 \pm 0.4$ & $1.8 \pm 0.3$ & $1.8 \pm 0.2$ & $1.6 \pm 0.4$ \\
\hline Right & WT & $4 / 0 \mathrm{~nm}$ & $3.2 \pm 0.8$ & & & $2.4 \pm 1.1$ \\
\hline Ventricle & $\mathrm{ChR} 2$ & 620 nm & $12 \pm 1.3$ & $7.6 \pm 1.6$ & & $10.2 \pm 1.2$ \\
\hline & WT & $0.30 \mathrm{~nm}$ & $12 \pm 2.2$ & & & $9.8 \pm 0.9$ \\
\hline
\end{tabular}

Table 6.1.: Light transmittance by the heart under different conditions. The heart and its components are enlisted in the main rows on the left side of the table from most to least light attenuating. On the rows next to them, ChR2-expressing hearts are separated from the wild type hearts and in the third column they are further divided into illuminated with either $470 \mathrm{~nm}$ or $625 \mathrm{~nm}$. On the columns the various conditions tested are ordered as tyrode had the highest transmittance of light the blood the least. The numbers represent the $\%$ of light detected by the photometer coming through the sample from the amount of light detected by the photometer without sample. ChR2 hearts with blood $(n=6)$, tyrode $(n=6)$, dye $(n=3)$, blebbistatin $(n=4)$. Wild type hearts with blood $(n=3)$, tyrode $(n=3)$.

and about 6-fold higher. This clearly supports the idea of using red-shifted opsins in order to increase the amount tissue excited.

Moreover, all the conditions tested affected light attenuation in a different way. The heart and its elements were most translucent when perfused only with Tyrode, which is important to keep in mind since ex vivo experiments are an important source of knowledge. When the dye was added, more light was absorbed and this can change the outcome of an experiment performed with or without optical mapping. Blebbistatin attenuated even more light than 
the dye, and hearts with blood in their chambers and circulation attenuated light the most. This behavior was observed for both wavelengths and for the whole heart, LV and septum, where dye, Blebbistatin and blood significantly increased attenuation compared to the Tyrode perfused.

Comparing the attenuation by blood, it was at least 2-fold higher than the tyrode solution for all hearts elements except for the RV. Moreover, illuminating a wild type mouse heart or ChR2 mouse heart with $625 \mathrm{~nm}$ did not make a difference in the transmitted light. On the other hand, illumination using $470 \mathrm{~nm}$ saw a clear drop in transmittance in the case of ChR2 mice hearts compared to the hearts without the channel, reflecting the absorption by the channels.

Considering measurements of the LV wall thickness $(x=1.79 \mathrm{~mm})$ obtained via magnetic resonance [73] in experiments from a different group, we can use the Lambert-Beer law to calculate attenuation coefficients for the ventricle under the different conditions tested as follows:

$$
\mu=\ln (1 / T(x)) / x
$$

with a wall thickness of $\mathrm{x}=1.79 \mathrm{~mm}$ and $\mathrm{T}(\mathrm{x})$ from Table 6.1 Results are shown in Table 6.2. Naturally, the highest attenuation coefficients belong to the conditions with the smallest transmittance. These results provide an indication on what to expect if the ChR2-mouse heart were optogenetically stimulated under these circumstances. Therefore the next step was to measure the response using different illumination parameters.

\begin{tabular}{|c|c|c|c|c|c|c|}
\hline \multicolumn{3}{|c|}{$\begin{array}{l}\text { attenuation coefficient }(\mu) \\
{[1 / \mathrm{mm}]}\end{array}$} & Tyrode & Dye & Blebbistatin & Blood \\
\hline \multirow{4}{*}{$\begin{array}{c}\text { Left } \\
\text { Ventricle }\end{array}$} & $\mathrm{ChR} 2$ & \multirow{2}{*}{$470 \mathrm{~nm}$} & 3.29 & 3.44 & 3.55 & 3.73 \\
\hline & WT & & 2.85 & & & 3.41 \\
\hline & $\mathrm{ChR2}$ & \multirow{2}{*}{$630 \mathrm{~nm}$} & 1.82 & & & 2.17 \\
\hline & WT & & 1.82 & 2.09 & & 2.21 \\
\hline
\end{tabular}

Table 6.2.: Calculated attenuation coefficient $\mu$ of the $\mathbf{L V}$ under different conditions. On the rows, ChR2-expressing hearts are separated from the wild type hearts and in the third column they are further divided into illuminated with either $470 \mathrm{~nm}$ or $625 \mathrm{~nm}$. On the columns the various conditions tested are ordered as tyrode had the highest transmittance of light the blood the least. 


\subsubsection{The Effect of Di-4-ANBDQPQ and Blebbistatin on the pacing thresholds of the right and left ventricles}

After exploring the differences in light attenuation under different conditions we investigated how these potentially affect the ability to optogenetically pace the heart. Therefore, we tested the minimum intensities required to pace the heart with at least 10 pulses in a row when illuminating the RV and the LV with an optical fiber of $d=400 \mu \mathrm{m}$ (Fig. 6.1). We also compared stimulating hearts with and without bolus injection of the potentiometric dye Di-4ANBDQPQ. In order to do this, a train of 20 pulses was used to illuminate the heart and if the last 10 responded consecutively for a specific intensity, then the intensity would be decreased until the minimum for that specific heart was found. Using the lowest intensity, the same train of pulses would be applied for a total of 5 times before establishing it as reproducible. Afterwards, either the dye or blebbistatin would be injected to the heart before performing the same measurements again, as described in Section 5.3 .

As shown in Figure 6.3 A, the intensity required to pace the heart was lower for the RV than for the LV for both pulse lengths and conditions (with and without Dye) tested. For the $5 \mathrm{~ms}$ pulse case, the $\mathrm{LV}$ required $4 \pm 0.9 \mathrm{~mW} / \mathrm{mm}^{2}$, compared to $2.4 \pm 0.8 \mathrm{~mW} / \mathrm{mm}^{2}$ on the $\mathrm{RV}$ (mean \pm s.d.). Furthermore, a significant increase in intensity was necessary to pace the Dye-injected heart on the $\mathrm{LV}$, jumping to $10.3 \pm 4.2 \mathrm{~mW} / \mathrm{mm}^{2}$. On the RV the increase was less pronounced, up to $3.6 \pm 1.3 \mathrm{~mW} / \mathrm{mm}^{2}$ for the $5 \mathrm{~ms}$ case.
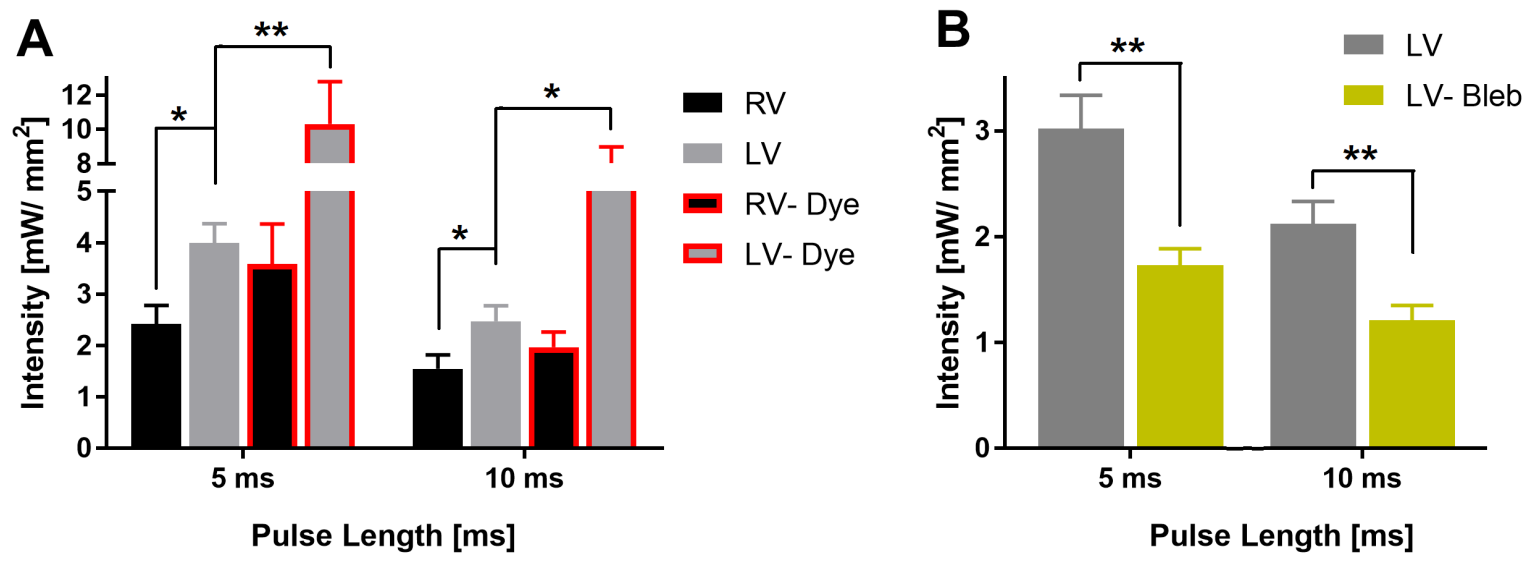

Figure 6.3.: Pacing under different conditions. The pacing threshold of the RV was lower than the threshold of the LV. Even though shorter pulses demanded higher intensities, the effect of the dye and of blebbistatin was alike for both pulse lengths; while (A) the dye increased the mean optogenetic pacing threshold, (B) blebbistatin decreased it. Plots shown as mean \pm s.e.m. $N=6$ for control hearts and hearts with blebbistatin, $N=3$ hearts with dye. Performed unpaired t-test $(* * \mathrm{p}<0.01$, $* \mathrm{p}<0.05)$.

Next, we checked the differences when pacing the LV before and after adding Blebbis- 
tatin to the perfusion solution. After 20-25 min of Tyrode-Blebbistatin perfusion, a decrease in the pacing threshold intensity was observed. Both $5 \mathrm{~ms}$ and $10 \mathrm{~ms}$ pulses required about $50 \%$ less intensity in the blebbistatin case (Figure 6.3 B). Since a tyrode-blebbistatin perfused LV transmitted a smaller amount of light than a tyrode-only perfused heart (Table 6.1), one could expect a higher stimulation threshold. I speculate that I observed the opposite, there could be a rather electrophysiological effect origin than a physical one, deserving special consideration when planning experiments. This will be further discussed in the Discussion \& Conclusions section.

\subsubsection{Pulse length, intensity and surface area determine the optogenetic pacing threshold}

In order to examine the effects of surface area, pulse length and intensity in optogenetic pacing we used two optical fibers with different diameters ( $400 \mu \mathrm{m}$ and $1000 \mu \mathrm{m}$ ) to pace the heart on the left ventricle at four different intensities. For each intensity the capture rate of the last 10 out of a train of 20 pulses at $9 \mathrm{~Hz}$ was measured for pulse widths $1 \mathrm{~ms}$ to $15 \mathrm{~ms}$. The plots in Figure 6.4 display the response of the heart to the different intensity/pulse width combinations for each fiber.
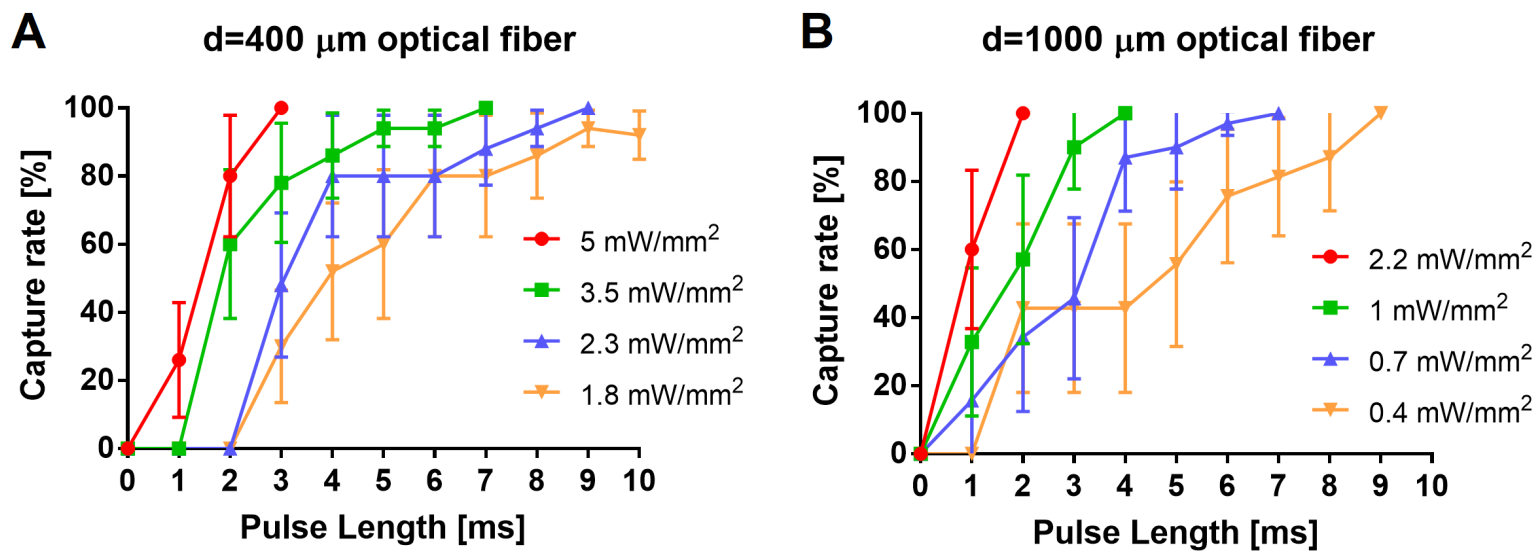

Figure 6.4.: Pacing with different surface areas. The response rate was step-wise [ms] tested for (A) $\mathrm{d}=400 \mu \mathrm{m}$ and $(\mathbf{B}) \mathrm{d}=1000 \mu \mathrm{m}$ at four different intensities and they both displayed the same tendency. Higher intensities reach 1:1 capture at shorter pulses. Each data point represents the mean \pm s.e.m. $N=5$ for $\mathrm{d}=400 \mu \mathrm{m}$ and $N=4$ for $\mathrm{d}=1000 \mu \mathrm{m}$.

As expected, both cases show similar behavior, with the highest intensities achieving 1:1 capture rate for shorter pulses and the lowest intensities requiring longer pulses in order to obtain a reproducible response (Figure 6.4). Another behavior in common is the variability in each data point. This can be explained as follows; it was expected that for each pulse width/intensity combination each heart would achieve a certain number of captures and we 
would therefore obtain a mean value representing all of the hearts. However, opposite to this, some hearts responded to $100 \%$ of the pulses while other responded to no pulse, leading to the variability.

Figure 6.5 plots for both surface areas probed the combinations of pulse widths and intensities that led to a 1:1 capture rate in all the hearts tested. In addition, the energy of these were also computed and plotted using dashed lines. For both cases, increases in pulse length allow a decrease in intensity, and vice versa. Similarly, for the same pulse width $(7 \mathrm{~ms})$, illuminating larger areas allows a decrease in intensity. And, when the same intensity was used $\left(\sim 2.2 \mathrm{~mW} / \mathrm{mm}^{2}\right)$ the fiber with the smaller diameter needed a $9 \mathrm{~ms}$ pulse compared to a $2 \mathrm{~ms}$ pulse when using the larger fiber.

The amount of energy delivered to the heart during optogenetic pacing remained somewhat constant for each fiber with an average of $2.8 \pm 0.5 \mu \mathrm{J}$ for the smaller fiber and $3.4 \pm$ $0.4 \mu \mathrm{J}$ for the larger one. Since pulse width and intensity compensate each other, an increase in energy with an increase in surface area illuminated is expected.

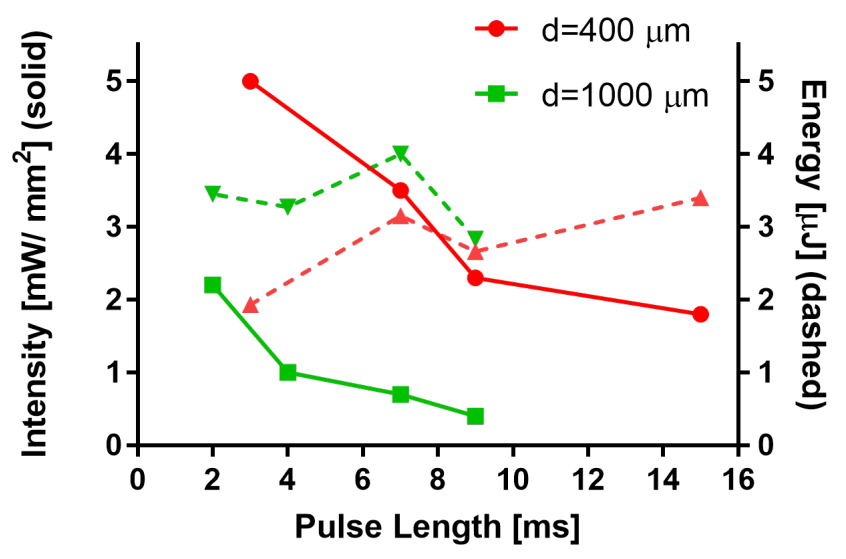

Figure 6.5.: Intensities and energies during optogenetic pacing with optical fibers. The intensity (solid lines) required to achieve 1:1 capture in all the hearts tested is reduced for the larger surface area. However, they both show a decrease in intensity for longer pulses. On the other hand, the energy (dashed lines) doesn't show a clear trend. $N=5$ for $\mathrm{d}=400 \mu \mathrm{m}$ (in red) and $N=4$ for $\mathrm{d}=1000 \mu \mathrm{m}$ (in green).

\subsection{Discussion \& Conclusions}

In optogenetics there are two key players: opsins which are the actuators, and light which is the triggering energy activating the actuators. With a deficiency in any of them optogenetic control could be hindered. Light attenuation is affected by the components of the heart as well as by laboratory elements used in cardiac research. For these reasons, it is of 
great importance to understand how the conditions of the heart in an experiment affect the light being delivered to it. In the same line, comparing the attenuation of these conditions for two different wavelengths and for each area of the heart can help in the design of future experiments and devices towards the application of cardiac optogenetics in the clinic.

To the best of our knowledge, this is the first optical transmittance investigation comparing the attenuating effects of solutions used in experiments and blood. Zaglia and colleagues measured penetration of blue light into the ventricular tissue, offering an estimate of light attenuation coefficient of $1 / 240 \mu \mathrm{m}^{-1}=4.16 \mathrm{~mm}^{-1}$, which is close to our attenuation coefficient obtained from the LV with blood of $3.73 \mathrm{~mm}^{-1}$ [96]. Their results also display that pacing the left ventricle requires a greater amount of light than for the right ventricle. As expected, our results show that penetration of red light was clearly greater than for the blue light. Also, comparing the absorption of both wavelengths in tyrode and blood, the ratio of increased attenuation in blood was always higher in the case of the blue light, which as mentioned in the introduction, is explained by the higher absorption coefficient of blood to this wavelength [71].

The results regarding transmittance and attenuation coefficient obtained in this subsection cannot be straightforward translated to calculate the differences in intensity thresholds to stimulate the ChR2-mice hearts since there are different factors playing a role such as concentrations of the dye and blebbistatin, the fluorescence effect of blebbistatin as well as electrophysiology of the stimulated cells. However, the results can provide an orientation on to which degree and which solutions attenuate light the most. More specifically for the mouse model heart used during this project and under the laboratory conditions of the following experiments.

An interesting point to highlight is how the dyes and blebbistatin can diminish the light delivered to the photo-sensitive channels. Both increased the coefficient of attenuation of the heart. However, while the stimulation threshold increased, as anticipated, with the dye, it decreased with blebbistatin. Blebbistatin has been shown to be inactivated with blue light [74] and also to show fluorescence and photo-toxic effects in single cells and tissue cultures [46], explaining the observed increase in absorption in our experiments. Despite a lack of an agreement on the effects of blebbistatin, some groups have reported alterations on the electrophysiology of various heart models [10, 83], such as shortening of action potential duration in rabbit hearts [40]. The evidence shown here might not be enough to prove that blebbistatin is affecting the sensitivity of optogenetically paced mice hearts, but optogenetics provides a new tool to design further experiments that could lead to a better understanding of the electromechanical uncoupler. A decrease in the pacing threshold intensity is not expected from a light attenuating substance. Researchers should take into account all the above mentioned factors related to the voltage-dye and blebbistatin at the moment of translating the results obtained using the optical mapping technique, since it is an important source of experiments in all-optical setups [63].

Lastly, our results regarding the roles of intensity, pulse length and surface area in opto- 
genetic pacing are in accordance with previous experiments [19, 96, 60]. The three parameters compensate each other, namely, you can increase/decrease one by complementing with one or the other two in order to obtain the same response. Therefore, short pulses will demand higher intensities in order to pace the heart and larger areas of stimulation will require lower intensities and/or shorter pulses to obtain a 1:1 capture rate. The advantage of illuminating larger areas will be especially favorable during the next chapter since optogenetic arrhythmia termination will be tackled by employing global photostimulation.

The results obtained from this first steps offer knowledge about simple optogenetic stimulation providing the necessary foundations before moving on to more complex scenarios such as arrhythmia termination. Moreover, the majority if not all of the results obtained with the mouse model can offer useful perception on what to expect from larger animal models or even humans.

\subsection{Contributions}

Part of the experiments were performed by the master students Tsima Abou Kors and Vishalini Vinkatesan during their master thesis projects. I completely designed and supervised the project carried on by Tsima, and I partially designed and supervised the project carried on by Vishalini. 



\section{Global Optogenetic Stimulation to Terminate Arrhythmias}

\subsection{Introduction and Aim}

After characterizing the effects of optogenetic stimulation on the murine heart, it was possible to use this results in order to translate them to a different task: optogenetic cardioversion. This chapter describes the most important part of this $\mathrm{PhD}$ work, which lead to a publication. At the moment of working on this project, all the optogenetic cardioversion approaches used local stimulation in order to terminate arrhythmias [12, 19, 61].

As described before, when using optogenetic stimulation pulse length, light intensity and the surface area of illumination play a role in the efficiency of the stimulus. Therefore, we thought that simultaneously illuminating the whole cardiac surface would lead to a decrease in one or both pulse length or light intensity used to terminate arrhythmias compared to the previous methods used. Moreover, up to a certain point it would mimic electrical defibrillation, where the whole heart is shocked.

Therefore, in order to accomplish the task of illuminating the whole epicardial illumination, which was the main difference of this method compared to the other groups, I designed an experimental setup that could synchronously stimulate globally the heart and record optical mapping data. The setup will be briefly discussed here before attaching the publication generated. On the part of the analysis, I also focused on how the arrhythmias were terminated, a feature not investigated before. By computing the time it takes the stimulation to terminate the arrhythmia I was able to have a better understanding of the mechanisms behind, and this is also discussed in the paper.

In summary, the aim of this chapter which is mainly composed of a paper, is to describe global optogenetic illumination as a method to terminate arrhythmias. From its characterization, to its advantages, disadvantages and mechanisms. The first step was to build the setup. 


\subsection{Experimental setup for global illumination}
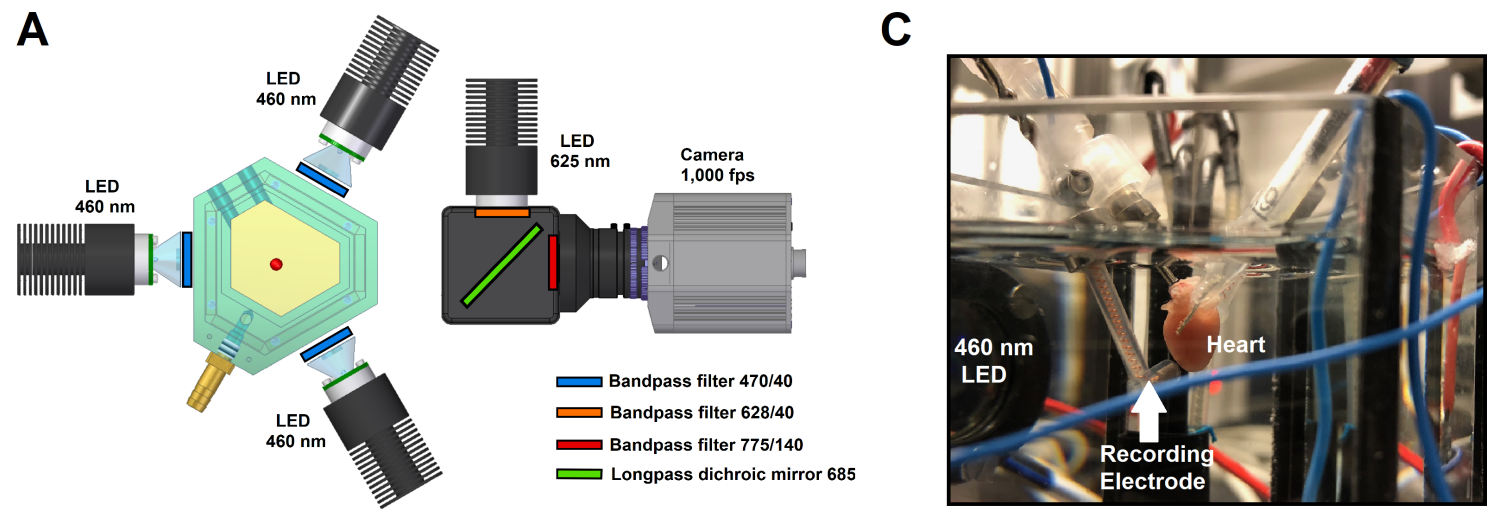

B

D
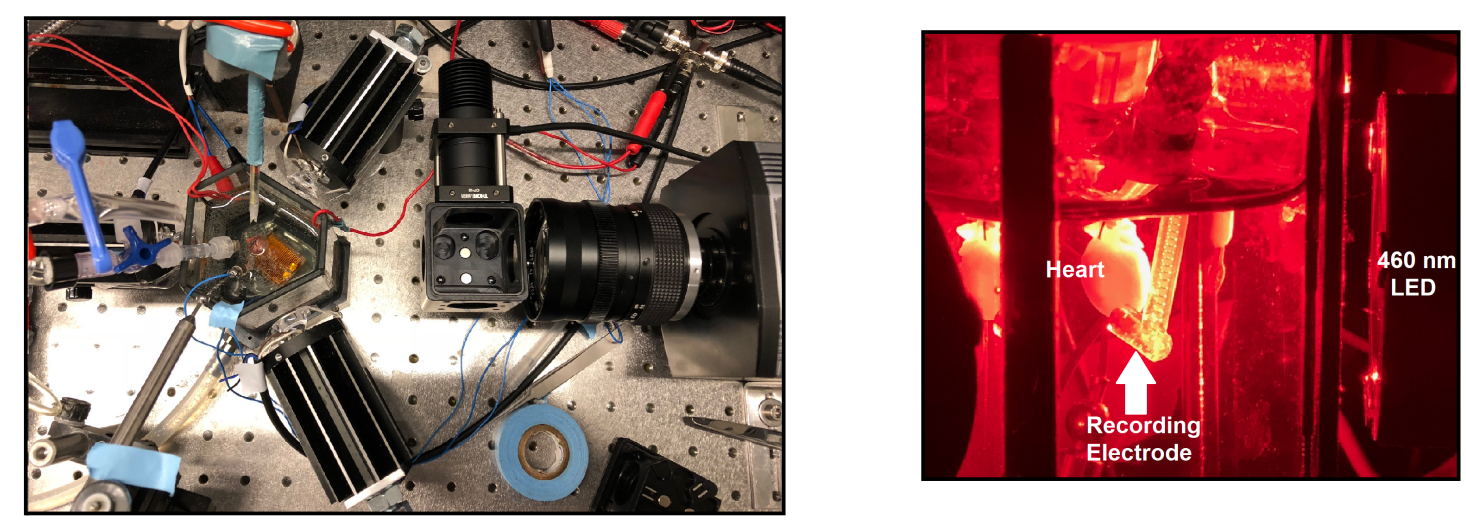

Figure 7.1.: Global Illumination Setup. (A) Sketch of the setup and all its components. On the left side the bath surrounded by the three blue-light LEDs and their filters. On the right side the imaging part composed of the LED to excite the dye as well as the dichroic mirror and the filter of the emission light coming from the heart. (B) Photo of the setup placed in the same position as (A). (C) Lateral view of the bath with the heart vertically arranged. On the left side of the photo the apex of the heart is in contact with the MAP electrode to record the electrical signal. (D) Lateral view of the heart in the bath. This time the excitation light of the dye is irradiating the bath. On the right side of the photo one of the blue LEDs can be appreciated, with its diameter larger than the height of the heart.

In order to investigate for the first time the effect of global optogenetic stimulation of the ChR2 mouse heart, this setup had to be built. Since the arrhythmias induced were located in the ventricles it was a priority to illuminate both ventricles completely with the same intensity. Hence, using a custom-built hexagonal perfusion bath that would allow vertical positioning of the heart (Fig.7.1 C) we were able to surround the heart with three illumination sources (Fig.7.1 C) that would simultaneously illuminate the right and the left ventricles and part of the atria. In addition, one side of the bath had a clear view over the heart in order to perform 
optical mapping measurements.

The stimulation part of the setup was composed of three blue LEDs equally arranged and distanced around the bath. Before reaching the heart, the light irradiated from the LEDs was filtered in order to discriminate other wavelengths that could interfere with the optical mapping. The imaging part of the setup was composed of red-light mounted LED to excite the voltage dye. This light was also filtered before reaching a longpass dichroic mirror that would direct the light to the heart and prevent blue light from the stimulation to get in the path of the camera.

Precisely, the setup was composed of:

- The perfusion bath.

- Three high power LEDs with the wavelength centered at $460 \mathrm{~nm}$ (Led Engin, LZ400B208), lenses and bandpass filters 470/40 (Chroma, F49-470).

- Mounted LED $625 \mathrm{~nm}$ with a bandpass filter 628/40 (Semrock).

- Longpass dichroic mirror at $685 \mathrm{~nm}$ (Semrock).

- Bandpass filter 775/140 nm (Semrock).

- EMCCD camera (Cascade128+, Photometrics)

As described in the manuscript attached in the following section, after the setup was built the first steps consisted of characterizing the excitation using global illumination, characterize arrhythmia induction, investigate the termination of arrhythmias applying stimuli of different pulse lengths, analyze the termination time of arrhythmias and the electric phenomena taking place on the surface of the heart during optogenetic arrhythmia termination using optical mapping. All of this is described on the next chapter composed of the publication.

\subsection{Results (publication)}

In this section you can find attached the publication "Energy-Reduced Arrhythmia Termination Using Global Photostimulation in Optogenetic Murine Hearts" in the journal Frontiers in Physiology. My contribution for this paper was the design, performance and analysis of all the experiments, as well as writing the paper together with my co-authors.

The Results obtained are the following, and can be found in page 5 of the attached paper: 
7. Global Optogenetic Stimulation to Terminate Arrhythmias

\subsubsection{Global vs. Local Optogenetic Pacing}

Found in Section 3.1 of the attached paper.

\subsubsection{Global Optogenetic Cardioversion}

Found in Section 3.2 of the attached paper.

\subsubsection{Effects of Global Illumination on Arrhythmia Patterns}

Found in Section 3.3 of the attached paper. 
OPEN ACCESS

Edited by:

Ming Lei,

University of Oxford, United Kingdom

Reviewed by:

Alexey V. Glukhov,

University of Wisconsin System,

United States

Jason D. Bayer

Université de Bordeaux, France

*Correspondence:

Claudia Richter

claudia.richter@ds.mpg.de

Specialty section:

This article was submitted to

Cardiac Electrophysiology,

a section of the journal

Frontiers in Physiology

Received: 13 August 2018 Accepted: 02 November 2018 Published: 27 November 2018

Citation:

Quiñonez Uribe RA, Luther $S$

Diaz-Maue L and Richter C (2018)

Energy-Reduced Arrhythmia

Termination Using Global

Photostimulation in Optogenetic

Murine Hearts. Front. Physiol. 9:1651.

doi: 10.3389/fphys.2018.0165

\section{Energy-Reduced Arrhythmia Termination Using Global Photostimulation in Optogenetic Murine Hearts}

\author{
Raúl A. Quiñonez Uribe ${ }^{1}$, Stefan Luther ${ }^{1,2,3,4}$, Laura Diaz-Maue ${ }^{1}$ and Claudia Richter ${ }^{1,4,5 *}$ \\ ${ }^{1}$ RG Biomedical Physics, Max Planck Institute for Dynamics and Self-Organization, Göttingen, Germany, ${ }^{2}$ Institute for \\ Nonlinear Dynamics, Georg-August University, Göttingen, Germany, ${ }^{3}$ Department of Pharmacology and Toxicology, \\ University Medical Center, Göttingen, Germany, ${ }^{4}$ German Center for Cardiovascular Research (DZHK e.V.), Partner Site \\ Göttingen, Göttingen, Germany, ${ }^{5}$ Department of Cardiology and Pneumology, University Medical Center, Göttingen, Germany
}

Complex spatiotemporal non-linearity as observed during cardiac arrhythmia strongly correlates with vortex-like excitation wavelengths and tissue characteristics. Therefore, the control of arrhythmic patterns requires fundamental understanding of dependencies between onset and perpetuation of arrhythmia and substrate instabilities. Available treatments, such as drug application or high-energy electrical shocks, are discussed for potential side effects resulting in prognosis worsening due to the lack of specificity and spatiotemporal precision. In contrast, cardiac optogenetics relies on light sensitive ion channels stimulated to trigger excitation of cardiomyocytes solely making use of the inner cell mechanisms. This enables low-energy, non-damaging optical control of cardiac excitation with high resolution. Recently, the capability of optogenetic cardioversion was shown in Channelrhodopsin-2 (ChR2) transgenic mice. But these studies used mainly structured and local illumination for cardiac stimulation. In addition, since optogenetic and electrical stimulus work on different principles to control the electrical activity of cardiac tissue, a better understanding of the phenomena behind optogenetic cardioversion is still needed. The present study aims to investigate global illumination with regard to parameter characterization and its potential for cardioversion. Our results show that by tuning the light intensity without exceeding $1.10 \mathrm{~mW} \mathrm{~mm}^{-2}$, a single pulse in the range of $10-1,000 \mathrm{~ms}$ is sufficient to reliably reset the heart into sinus rhythm. The combination of our panoramic low-intensity photostimulation with optical mapping techniques visualized wave collision resulting in annihilation as well as propagation perturbations as mechanisms leading to optogenetic cardioversion, which seem to base on other processes than electrical defibrillation. This study contributes to the understanding of the roles played by epicardial illumination, pulse duration and light intensity in optogenetic cardioversion, which are the main variables influencing cardiac optogenetic control, highlighting the advantages and insights of global stimulation. Therefore, the presented results can be modules in the design of novel illumination technologies with specific energy requirements on the way toward tissue-protective defibrillation techniques.

Keywords: optogenetics, energy-reduced defibrillation, cardiac arrhythmia, channelrhodopsin-2, photostimulation, global illumination 


\section{Global Optogenetic Stimulation to Terminate Arrhythmias}

\section{INTRODUCTION}

Spatiotemporal dynamics in biological systems, particularly the control of complex excitation patterns, are a fundamental nonlinear problem with extensive potential in medical engineering and therapeutic application. Due to the intrinsic complexity of cardiac tissue, it is challenging to understand in detail the underlying biophysical mechanisms of arrhythmia. The normal sinus rhythm of the heart is triggered by regular, quasiplanar waves of electric depolarization. Spatiotemporally chaotic activation patterns have been identified, and are shown to be responsible for arrhythmic, life-threatening regimes (Davidenko et al., 1992; Luther et al., 2011; Christoph et al., 2018). The inferences of patterns in electrocardiograms (ECG), which showed up as irregular, sometimes aperiodic structures, gave the impulse to think of arrhythmia and especially of ventricular fibrillation as an uncontrolled, shivering activation of heart muscle. Thereby the underlying patterns are results of multiple erratic excitation waves changing in direction and shape. The complexity of wave patterns, leading to spatiotemporal chaotic regimes, is a consequence of the non-linearity. The dynamical processes are characterized by the annihilation of interacting waves, a mechanism also found in other physical systems (Panfilov and Holden, 1990; Jalifé et al., 1998). State-of-the-art therapies include high energy electrical shocks applied either external or internal to defibrillate the heart. These, for the patient mostly painful, shocks terminate the chaotic spreading activity almost certainly, but are suspected to worsen the existing tissue conditions mostly due to their potential electroporating effect on cardiomyocytes. Hence, they also serve as trigger for new arrhythmia with increasing probability over time. Much work has been devoted to the search for improved therapies (see e.g., Zipes and Jalife, 2009). Methods such as Anti-Tachycardia Pacing (ATP), already used in implantable devices, involve very small electrical currents delivered by a single electrode. Provided that the pacing frequency is high enough, ATP can terminate arrhythmia with a fairly high success rate (Wathen et al., 2004). Regardless, even with a high success rate the case of failure can never be disesteemed. Especially stationery vortex-activities are difficult to terminate with only one pacing electrode, which is not close enough to the pinning region. So it is not astonishing that several research groups are investigating advanced implementations of ATP compared to the traditional applied defibrillation shocks (Efimov et al., 2000; Exner, 2005). In addition to the ATP, the Low-Energy Anti-Fibrillation Pacing (LEAP) method was announced. It consists in pacing the tissue with an externally applied electric field. In vitro and in vivo experiments have provided ample evidence that LEAP significantly reduces the energy necessary to terminate atrial and ventricular fibrillation (Fenton et al., 2009; Luther et al., 2011) by using repeated stimulation with fields of lower amplitude. One crucial feature of LEAP is that it is based on multiple virtual electrodes induced by intrinsic obstacles. Referring to former in vitro and in vivo experiments (Exner, 2005), especially defibrillation approaches implementing multiple pacing sites have significant influence on arrhythmia specific excitation patterns resulting in rapid synchronization. Anyhow, in order 62 to stimulate at multiple pacing sites either multiple implanted electrodes or specific electrical fields are necessary, which raises obvious translational hurdles. Also, all these valuable methods are still based on electrical shock application, which in turn can never be fully acquitted of potential worsening side effects. Consequentially, the evaluation of new cardiac treatments with side effect diminishing properties but fairly high success rates has to be brought into focus. At this point patterned light control of optogenetically modified cardiac tissue gives the opportunity to specifically stimulate well-defined tissue regions without critical Faraday reactions. Optogenetic photostimulation uses light of specific wavelengths to activate light-sensitive ion channels, which works without former electrically induced membrane potential changes (Bruegmann et al., 2011; Deisseroth, 2011). Recently, optogenetic cardioversion methods applying localized photostimulation were shown to be feasible (Zaglia et al., 2015; Bruegmann et al., 2016; Crocini et al., 2016; Nyns et al., 2016; Richter et al., 2016). Although much effort was put into the characterization of locally applied light intensity and energy (Bruegmann et al., 2010; Zaglia et al., 2015; Diaz-Maue et al., 2018) the underlying dependencies of light intensity, pulse duration and successful cardioversion remains somehow elusive.

However, recent studies showed that inducing multi-centered excitation within the arrhythmic tissue leads to a better control of spatiotemporal wave patterns, typical for fibrillation (Pumir et al., 2007; Luther et al., 2011; Janardhan et al., 2012). Having this in mind, successful global photostimulation would represent the maximum number of available pacing sites. In comparison with the conventional high-energetic electrotherapy, global photostimulation could overcome adverse side-effects like electroporation or unwanted co-stimulation of sensible neurons responsible for pain sensation during defibrillation. Indeed there still remain some questions to be solved before global illumination or multi-site photostimulation could count for reliable defibrillation. With regard to potential clinical translation especially the dependencies between the minimal required light intensity and pulse duration as well as the applied over-all energy are important for the design and optimization of implanteable light-emitting devices. Furthermore, the investigation of global photodefibrilation and the underlying spatiotemporal mechanisms could help to deepen our understanding of the mode of action of conventional electrotherapy.

In the present study, we determine the threshold value of the applied global photostimulation as a function of the intensity and pulse duration, and we compare our experimental results with other photostimulation data. Another main question is whether it is possible to terminate arrhythmia like patterns by light-induced excitation.

\section{MATERIALS AND METHODS}

All experiments were done in accordance with the current version of the German animal welfare law and were reported to our animal welfare representatives; the application for approval of animal experiments has been approved by the responsible animal welfare authority (Lower Saxony State Office 
for Consumer protection and Food Safety). Humane welfareoriented procedures were carried out in accordance with the Guide for the Care and Use of Laboratory Animals and done after recommendations of the Federation of Laboratory Animal Science Associations (FELASA).

\subsection{Langendorff Perfusion}

The presented experiments are based on retrograde Langendorff perfusion using a constitutive transgenic mouse model, $\alpha$ MHC-ChR2, which restricts expression of channelrhodopsin2 (ChR2) to cardiac tissue. The ChR2 expression was proven by biomolecular protocols. The perfusion protocol includes two different tyrode solutions, either for arrhythmia induction or maintenance. The maintenance tyrode composition was described elsewhere (Richter et al., 2016). Briefly, $130 \mathrm{mM}$

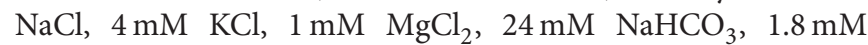
$\mathrm{CaCl}_{2}, 1.2 \mathrm{mM} \mathrm{KH}_{2} \mathrm{PO}_{4}, 5.6 \mathrm{mM}$ glucose, $1 \%$ albumin/BSA were aerated with carbogen ( $95 \%$ oxygen and $5 \% \mathrm{CO}_{2}$ ).

\subsubsection{Arrhythmia Induction}

In order to induce sustained arrhythmia we lowered the concentration of $\mathrm{KCl}$ to $2 \mathrm{mM}$, so that the arrhythmia induction tyrode contains $130 \mathrm{mM} \mathrm{NaCl}, 2 \mathrm{mM} \mathrm{KCl}, 1 \mathrm{mM} \mathrm{MgCl} 2,24 \mathrm{mM}$ $\mathrm{NaHCO}_{3}, 1.8 \mathrm{mM} \mathrm{CaCl}_{2}, 1.2 \mathrm{mM} \mathrm{KH}_{2} \mathrm{PO}_{4}, 5.6 \mathrm{mM}$ glucose, $1 \%$ albumin/BSA were aerated with carbogen $(95 \%$ oxygen and $\left.5 \% \mathrm{CO}_{2}\right)$. Because of a reduction in transmural dispersion of repolarization the so induced hypokalemia enhances arrhythmia induction (Killeen et al., 2007). In addition, $100 \mu \mathrm{M}$ Pinacidil, which is a KATPchannel activator, was applied to shorten the action potential duration (Wilde, 1994; Glukhov et al., 2010). The combination of both factors has been successfully applied to induce long sustained ventricular arrhythmia in murine Langendorff-perfused hearts (Bruegmann et al., 2016). Sustained arrhythmia was induced by applying 30 electrical pulses with a needle electrode in the range of $30-50 \mathrm{~Hz}$. To diminish motion artifacts in optical records, the contraction uncoupling reagent Blebbistatin $(c=5 \mu \mathrm{M}$, Thermo Fisher Scientific) was administrated. Potientiometric staining was achieved by the red-shifted dye Di-4-ANBDQPQ $(c=50 \mu \mathrm{M}$, Thermo Fisher Scientific) via bolus injection. All perfusion experiments were done at $37^{\circ} \mathrm{C}$.

\subsubsection{Optical Mapping}

A longpass $685 \mathrm{~nm}$ dichroic mirror (FF685-Di02-25x36, Semrock) was integrated to reflect the excitation light from a $625 \mathrm{~nm}$ mounted LED (M625L3, Thorlabs) after a bandpass filter (FF02-628/40-25, Semrock) onto the heart. The emission light was collected with a $775 \pm 70 \mathrm{~nm}$ bandpass filter (FF01775/140-25, Semrock) before reaching the camera (see Figure 1). Epicardial signal recording of the anterior wall was done with an electron multiplying charged coupled device (EMCCD, Cascade $128+$, Photometrics) camera with a spatial resolution of $64 \times 64$ pixels $(133 \mu \mathrm{m}$ per pixel) at $1 \mathrm{kHz}$. Camera control was achieved using custom-made recording software and the electrical heart activity was recorded using a monophasic action potential (MAP) electrode (BIOPAC Systems, Inc.).

\subsection{Optogenetic Illumination Strategies}

We employed local and global illumination to stimulate the heart, whereby only global illumination was used for cardioversion. Local illumination was achieved by positioning the tip of an optical fiber of $\varnothing=400 \mu \mathrm{m}$ in contact with the left ventricle. On the other hand, in order to achieve a consistent optogenetic stimulation of the whole heart surface and therewith global illumination, the hearts were vertically arranged surrounded by three blue-light emitting diodes (blue-LED, Thorlabs) with their wavelengths centered at $460 \mathrm{~nm}$ and limited by a $470 \pm 20 \mathrm{~nm}$ bandpass filter (ET470/40x, Chroma) (see Figure 1). Synchronous millisecond control of LED at different intensities was conducted via a function generator (Arbitrary Function Generator A2230, Agilent Instruments). Intensity measurements were done using the PM100D optical power meter and the S120VC photodiode power sensor (Thorlabs). Since the experimental setup consists of three blue-LED spaced at $120^{\circ}$, the intensity was measured directly facing each LED from the heart position and the calculated mean was considered the overall light intensity during global illumination.

To minimize effects of potential edema as well as metabolic changes during repeated arrhythmia periods on the defibrillation success rate, we limited the experimental time to $2 \mathrm{~h}$ and a maximum number of defibrillation attempts of 50 .

\subsection{Data Analysis}

The obtained fluorescent images were analyzed and processed using MatLab (MathWork, Inc.). Briefly, spatial and temporal smoothing filter were applied after pixelwise normalization. Overlapping of the blue light with the emission signal of the dye was removed by subtracting the average difference in intensities with and without blue light from each pixel over time (see Figure S1). The estimation of the total surface area of both ventricles and atria was achieved by reconstructing three-dimensional heart shapes from photographs using a shape from contour approach as previously described (Christoph et al., 2017). In total three hearts were used to calculate an average epicardiac surface area of $274 \mathrm{~mm}^{2}$. Statistical analysis was done by Student's $t$-test (one-tailored and unequal variance) comparing each increasing step with the following value. Throughout the text results are indicated with \pm standard deviation, unless otherwise noted.

Concerning local and global pacing experiments a train of 20 pulses was applied, whereby only the last 10 pulses were considered for the analysis. The minimum pulse lengths needed to reach 1:1 capture in all the tested hearts for different intensities were investigated. Each light intensity was tested in combination with maximal four different pulse duration values.

To determine the success rate of arrhythmia termination, we induced multiple arrhythmia in each heart and attempted termination 10 times with each light intensity-duration combination. To consider an arrhythmia as sustained we waited for $5 \mathrm{~s}$ after induction before attempting termination, which consisted of illuminating the whole heart by simultaneously turning on the three LED for the duration and intensity tested. If the arrhythmia stopped within maximum $1 \mathrm{~s}$ after the conclusion of stimulation, it was considered a successful attempt. In case 63 


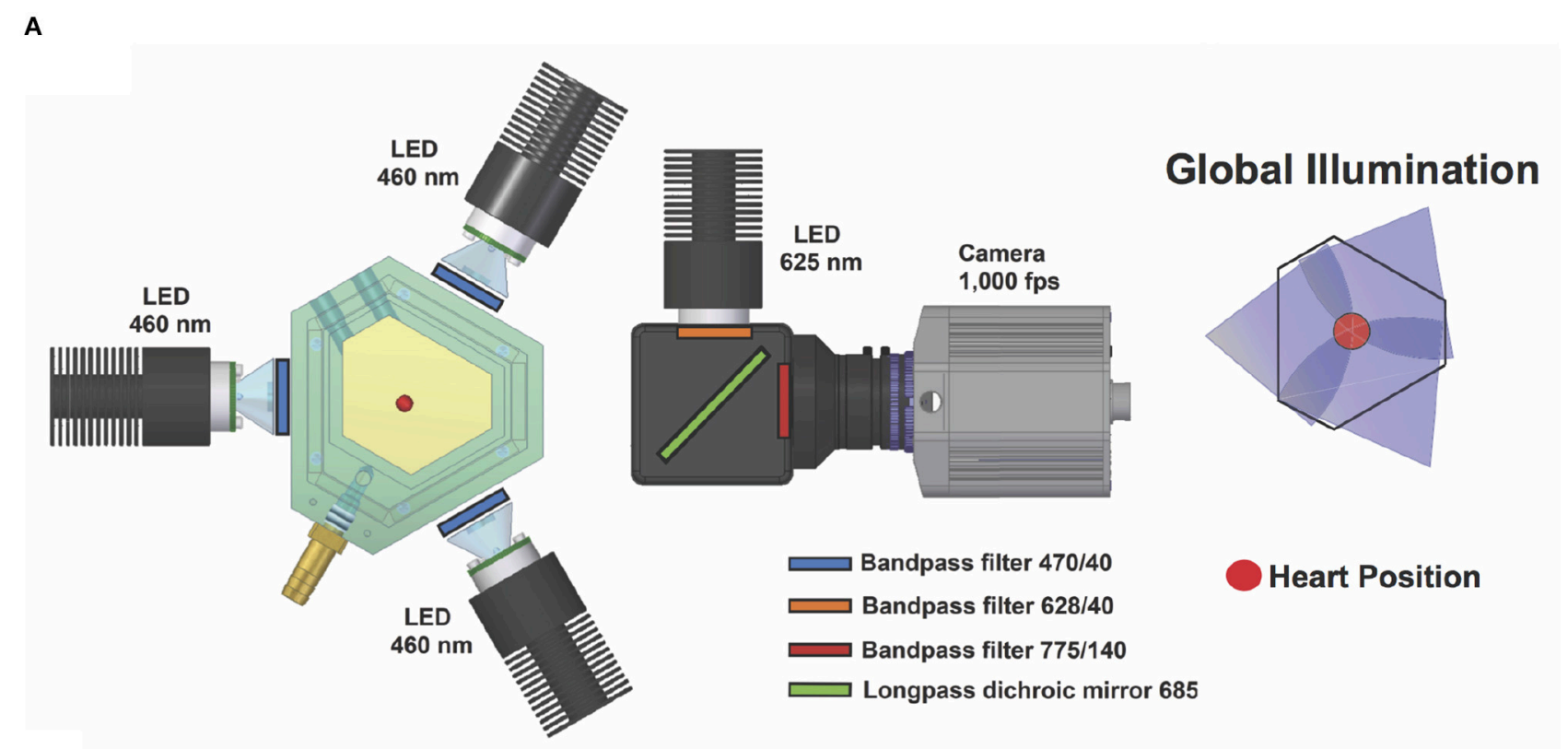

B

\section{Sinus Rhythm}
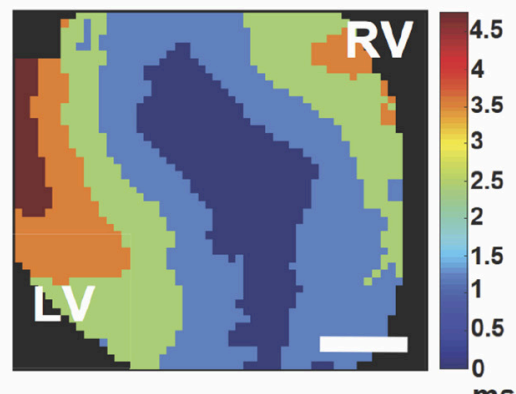

ms

C

\section{Spiral Activity}

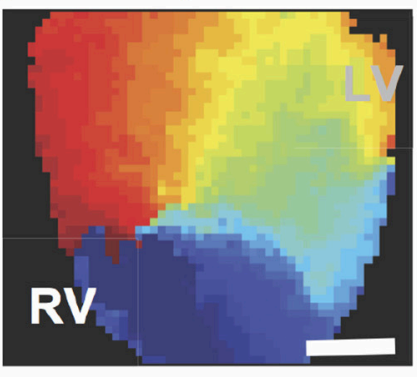

Electrical Pacing

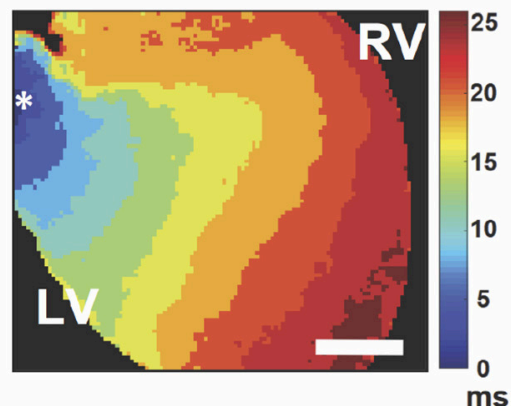

Photostimulation

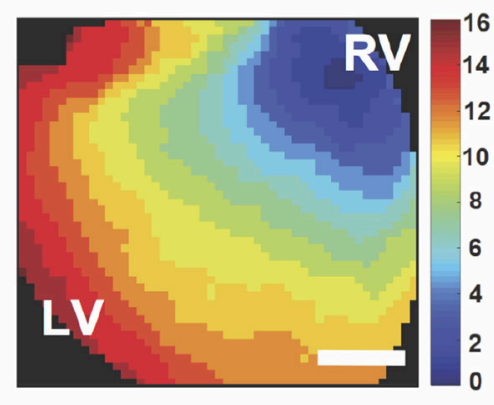

ms 


\subsection{Results (publication)}

of failed termination, we applied backup defibrillation, which consisted on increasing the intensity and duration of the pulses. To support the defibrillation procedure the hearts were perfused with maintenance tyrode (as described in section 2.1 and 2.1.1). The parameter combination with the highest termination rate and the lowest pulse duration values were determined as the most efficient ones. They also served as a decision-critical point for parameter change.

\section{RESULTS}

\subsection{Global vs. Local Optogenetic Pacing}

To characterize successful cardiac photostimulation, we tested the necessary pacing parameters to achieve 1:1 capture with respect to different pulse durations and light intensities. In order to avoid potential photochemical reactions and other side-effects stimulation light was turned off after every pacing.

First, global pacing was applied. Table 1 gives an overview of the step-wise measured intensities in relation to pulse duration and success rate. Our results prove that a shorter pulse duration correlates with higher intensities needed to gain 1:1 capture. The shortest pulse duration to successfully pace was $t_{\text {gpacing }}=$ $3 \mathrm{~ms}$ applying an intensity of $I_{\text {gpacing }}=113 \mu \mathrm{W} \mathrm{mm} \mathrm{m}^{-2}(N=$ 6). For pacing with $20 \mathrm{~ms}$ pulses successful pacing required an intensity of $I_{\text {gpacing }}=19.6 \mu \mathrm{W} \mathrm{mm} \mathrm{m}^{-2}$. Thus, constituting the lowest intensity reported to pace an optogenetic murine heart by photostimulation so far. Pacing the heart with $I_{\text {gpacing }}=$ $140 \mu \mathrm{W} \mathrm{mm} \mathrm{m}^{-2}$ was also tested for $t_{\text {gpacing }}=2 \mathrm{~ms}$, but could only be considered successful in one heart. Figure 2 shows the intensities and energies necessary to pace the heart with 1:1 capture at different pulse lengths using global stimulation. The graph shows that the energy necessary to pace at different intensity and duration combinations remained constant at an average of $E_{\text {gpacing }}=98 \pm 5 \mu \mathrm{J}$.

For comparison, we also conducted local photostimulation. Considering an increase in intensity required to pace the heart when stimulating smaller areas, we measured the intensity necessary to achieve 1:1 capture using an optical fiber of $A_{\text {fiber }}=0.126 \mathrm{~mm}^{2}$ with a $t_{\text {lpacing }}$ of $3 \mathrm{~ms}, 7 \mathrm{~ms}, 9 \mathrm{~ms}$ and $15 \mathrm{~ms}$. Compared to global pacing, all the intensity values required to obtain 1:1 capture were increased by minimum one order of magnitude with a maximum of $I_{\text {lpacing }}=1.77 \mathrm{~mW} \mathrm{~mm}^{-2}$.

In the course of these experiments, it could be observed that the average energy delivered to the epicardium is constant for both global and local illumination. In spite of the clear increase in intensity, it is in average 30 -fold lower when pacing locally than globally, at $E_{\text {lpacing }}=2.8 \pm 0.6 \mu \mathrm{J}$ and $E_{\text {gpacing }}=98 \pm 5 \mu \mathrm{J}$, respectively.

\subsection{Global Optogenetic Cardioversion}

In order to find the optimal parameters for optical cardioversion, we induced cardiac tachyarrhythmia applying Pinacidil and rapid electrical pacing. Therewith $75 \%$ of all arrhythmia lasted more than $t_{\text {arr }}=5 \mathrm{~s}$ (59 out of 79 induced arrhythmia). We considered the $t_{\text {arr }}=5 \mathrm{~s}$ duration as threshold for classification as sustained, since about $90 \%$ lasted for $t_{\text {arr }} \geq 10 \mathrm{~s}$. Non-sustained arrhythmia shorter than the threshold lasted on average $t_{\text {arr }}=$
$1.5 \pm 1.0 \mathrm{~s}$ (Figure 3 ). The average arrhythmia frequency was $f_{\text {arr }}$ $=24 \pm 5 \mathrm{~Hz}$, and one third of all induced arrhythmia lasted $t_{\text {arr }}$ $\geq 60$ s. Optical Mapping data showed multiple vortex-like wave dynamics, hence proving the successful induction of ventricular arrhythmia (Figure 1C).

\subsubsection{Optical Parameter Characterization}

Our measurements concerning global optogenetic photodefibrillation were triggered by Bruegmann et al. (2016), where local illumination cardioversion was first achieved by using an intensity of $I_{c v \text { (Brueg.) }}=0.40 \mathrm{~mW} \mathrm{~mm}^{-2}$ comprising a surface area of $A_{\text {heart(Brueg.) }}=143 \mathrm{~mm}^{2}$ with a $1 \mathrm{~s}$ pulse. In order to match the postulated amount of energy delivered by Bruegmann et al. $\left[E_{c v(\text { Brueg. })}=57.2 \mathrm{~mJ}\right]$ with our global illumination, which spans a larger epicardial surface $\left(A_{\text {heart }}=274 \mathrm{~mm}^{2}\right)$, we modulated the pulse duration by keeping the intensity constant at $I_{c v}=$ $0.42 \mathrm{~mW} \mathrm{~mm}^{-2}$. Figure 4 shows the main results of the photodefibrillation attempts. One single long stimulation pulse led to a successful arrhythmia termination in $82 \%$ of the experiments, which is comparable to the results obtained by Bruegmann et al.

Subsequently, we assessed the influence of light intensity by maintaining the pulse duration constant at $t_{c v}=500 \mathrm{~ms}$. While all tested light intensities successfully terminated the arrhythmia, a decrease in light intensity correlated with a significant depression in cardioversion efficiency (Figure 4B). At the most efficient light intensity of $I_{c v}=0.79 \mathrm{~mW} \mathrm{~mm}^{-2}$, a success rate of $96 \pm 2 \%$ [mean \pm standard error of the mean (SEM)] was achieved. However, a decrease in intensity by 40 -fold still managed to revert the heart rhythm in more than $30 \%$ of the attempts for the hearts tested $(N=6)$.

Afterwards, to evaluate the effect of pulse duration we kept the light intensity constant at $I_{c v}=0.79 \mathrm{~mW} \mathrm{~mm}^{-2}$ and applied pulses shorter than $t_{c v}=500 \mathrm{~ms}$. However, no significant change in termination rate was found for pulse durations of 500, 250, 100 , and $10 \mathrm{~ms}$. A shortening of pulse duration from 500 to $10 \mathrm{~ms}$ resulted in a decline of $13 \%$ of arrhythmia terminated (Figure 4B). Supposing the parameter combination of $t_{c v}=$ $10 \mathrm{~ms}$ and $I_{c v}=0.79 \mathrm{~mW} \mathrm{~mm}^{-2}$ is as efficient as $t_{c v}=$ $500 \mathrm{~ms}$ at the same light intensity, in the following we tested a constant pulse duration of $t_{c v}=10 \mathrm{~ms}$ while step-wise increasing light intensity from $I_{c v}=0.08$ to $1.1 \mathrm{mWmm}^{-2}$. Thereby we obtained successful termination in $92 \pm 4 \%$ of the experiments. Pursuing a wider comprehension of the effects of intensity and duration on global light-induced arrhythmia termination, we then experimented with $1 \mathrm{~s}$ long pulses, where an efficiency of $96 \pm 2 \%$ was achieved with $I_{c v}=0.56 \mathrm{~mW} \mathrm{~mm}^{-2}$.

\subsection{Effects of Global Illumination on Arrhythmia Patterns}

Since the $1 \mathrm{~s}$ stimuli lasts multiple tachycardia and sinus rhythm cycles, we identified that the transition from an arrhythmic state to the natural sinus frequency took place at different time points from the onset of stimulation. The electrical signal shown in Figures 4A,C shows a clear sinus activity even before the photostimulation has ended. To estimate the time between the beginning of stimulation and the moment of optical cardioversion, we defined two characteristic points in time of 65 


\section{Global Optogenetic Stimulation to Terminate Arrhythmias}

TABLE 1 | Measured pulse duration and light intensity combinations.

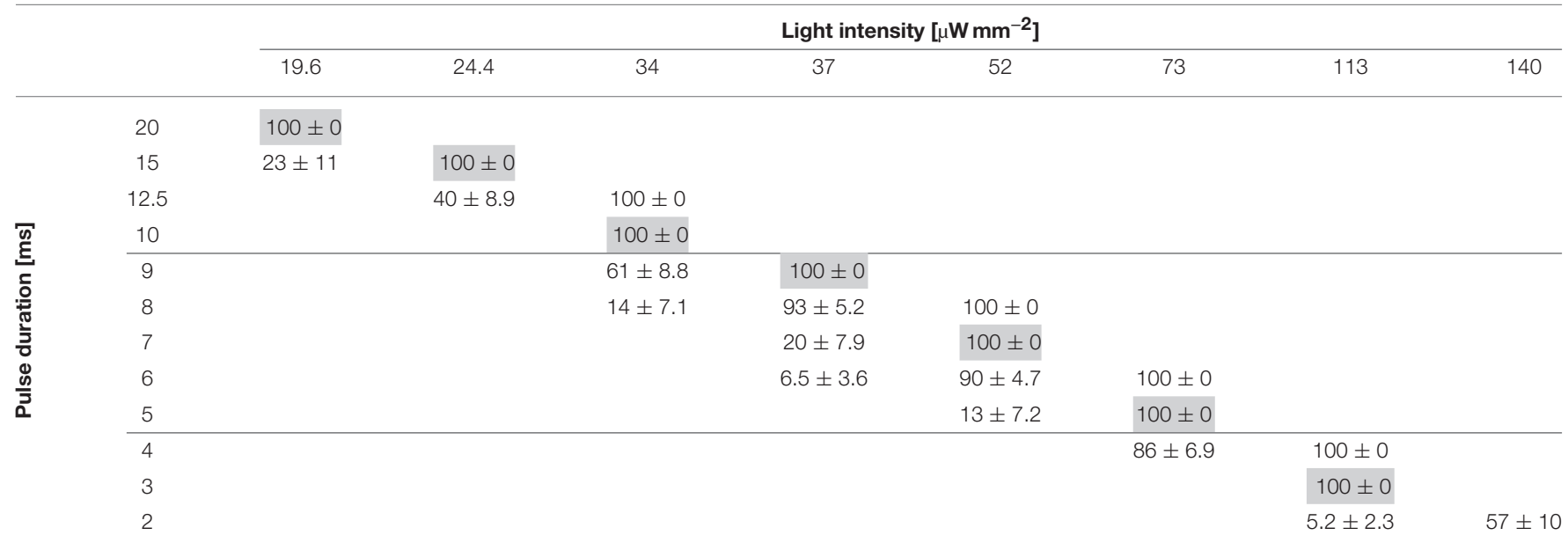

Inscribed is the averaged success rate [\%] \pm SEM of 1:1 capture during global illumination. The visible trend within the success rate regarding the dependency of light intensity and pulse duration is also mirrored in a constant energy level. Highlighted values indicate the most efficient pulse duration and light intensity combinations, which are drawn in Figure $\mathbf{2 B}$ together with the corresponding energy values. The shown values are based on 23 measurements from $N=6$ hearts.

the MAP signal (Figure 4C). First we define $t_{\text {last }}$ as the moment when the last peak of the arrhythmia occurred, and second $t_{\text {sin }}$ as the time point when the sinus rhythm signal first appeared. These two values were calculated for all the successful arrhythmia terminations of different intensities for the $1 \mathrm{~s}$ pulses. Figure 4D illustrates the percentages of $t_{\text {last }}$ and $t_{\text {sin }}$ for four time lapses. it can be observed that $t_{\text {last }}$ occurred at $\leq 60 \mathrm{~ms}$ of stimulus for $81 \%$ of the cardioversions when triggered with $I_{c v}=0.56 \mathrm{~mW} \mathrm{~mm}^{-2}$. However, this seems to depend on the light intensity, since cardioversions triggered with $I_{c v}=0.25 \mathrm{~mW} \mathrm{~mm}^{-2}$ showed this phenomenon in $55 \%$ of the cases and with an intensity of $I_{c v}=0.08 \mathrm{~mW} \mathrm{~mm}^{-2}$ in only $30 \%$. Furthermore, $t_{\text {sin }} \leq 215 \mathrm{~ms}$ occurred in 76,60 , and $54 \%$ of the respective tested intensities.

\subsubsection{Cardiac Dynamics During Global Illumination}

The optical mapping analysis of the $10 \mathrm{~ms}$ as well as $1 \mathrm{~s}$ stimulation experiments combined with the analysis of $t_{\text {last }}$ in $1 \mathrm{~s}$ pulse attempts led to identify two preferential mechanisms for global optical cardioversion. The first mechanism consists of a cardioversion that happens on the onset of illumination, mainly annihilating the spiral by the depolarization and following refractoriness of the cardiac tissue (Figure 5). While in the second mechanism, an unpinning and disturbance of the spiral can be observed (Figure 6), with the elimination of the spiral taking place at the mid and late stimulation period. Optical mapping data showing the two mechanisms could be found in the (Supplementary Material Movies 2, 3). As the dominant mechanism the annihilation was observed for both pulse duration $10 \mathrm{~ms}$ and $1 \mathrm{~s}$, and took place in $90 \%$ of all cardioversions using $1 \mathrm{~s}$ pulses $(N=120)$. This mechanism included a $t_{\text {last }}=58 \pm 19 \mathrm{~ms}$. In contrast, the second mechanism was only observed in the $1 \mathrm{~s}$ pulses with an averaged $t_{\text {last }}=$ $296 \pm 122 \mathrm{~ms}$. Analyzing the MAP recordings led to a clear distinction between the two mechanisms, whereby the number of arrhythmic excitation peaks during $t_{\text {last }}$ was $\leq 2$ for $86 \%$ of 66 all the annihilation observations, while all the unpinning cases presented $>4$ arrhythmia cycles during $t_{\text {last }}$.

\section{DISCUSSION}

The control of spatiotemporal cardiac regimes and the influence of the thereto applied electrical pulses have been investigated extensively in theory and experiment in the last decades (see e.g., Pumir and Krinsky, 1999; Takagi et al., 2004). In consequence, especially the diminishment of adverse side effects of high-energy electrical shocks, like e.g., electroporatic cell membrane disturbances, while keeping a very high success rate has been highly prioritized. In this manner, Janardhan et al. introduced a low-energy defibrillation approach to successfully terminate ventricular tachycardia (VT) applying multistage organized shocks (Janardhan et al., 2012). Thereby they eliminated the phase dependence of shock application, which is crucial for single shock therapy in VT. Furthermore, the possibility to control cardiac excitation dynamics by applying multi-centered new activation origins was proposed as one auspicious approach (Pumir et al., 2007; Luther et al., 2011). Luther et al. impressively showed that recruiting multiple pacing sites has remarkable success in countersteering arrhythmic regimes, which in return makes tissue protective defibrillation feasible. Against this background, nonelectrical approaches would benefit both the development of tissue protective defibrillation and the investigation of mechanistic associations without unpredictable worsening side effects. Cardiac optogenetics with its light initiated depolarization and accordingly hyperpolarization fulfills this position as a suitable tool to characterize mechanisms underlying multisite pacing. Considering the complex non-linear dynamics of cardiac arrhythmia, photostimulation convinces with the highly controllable temporal as well as spatial resolution. Compared to conventional electrical approaches, the direct interaction of 


\subsection{Results (publication)}

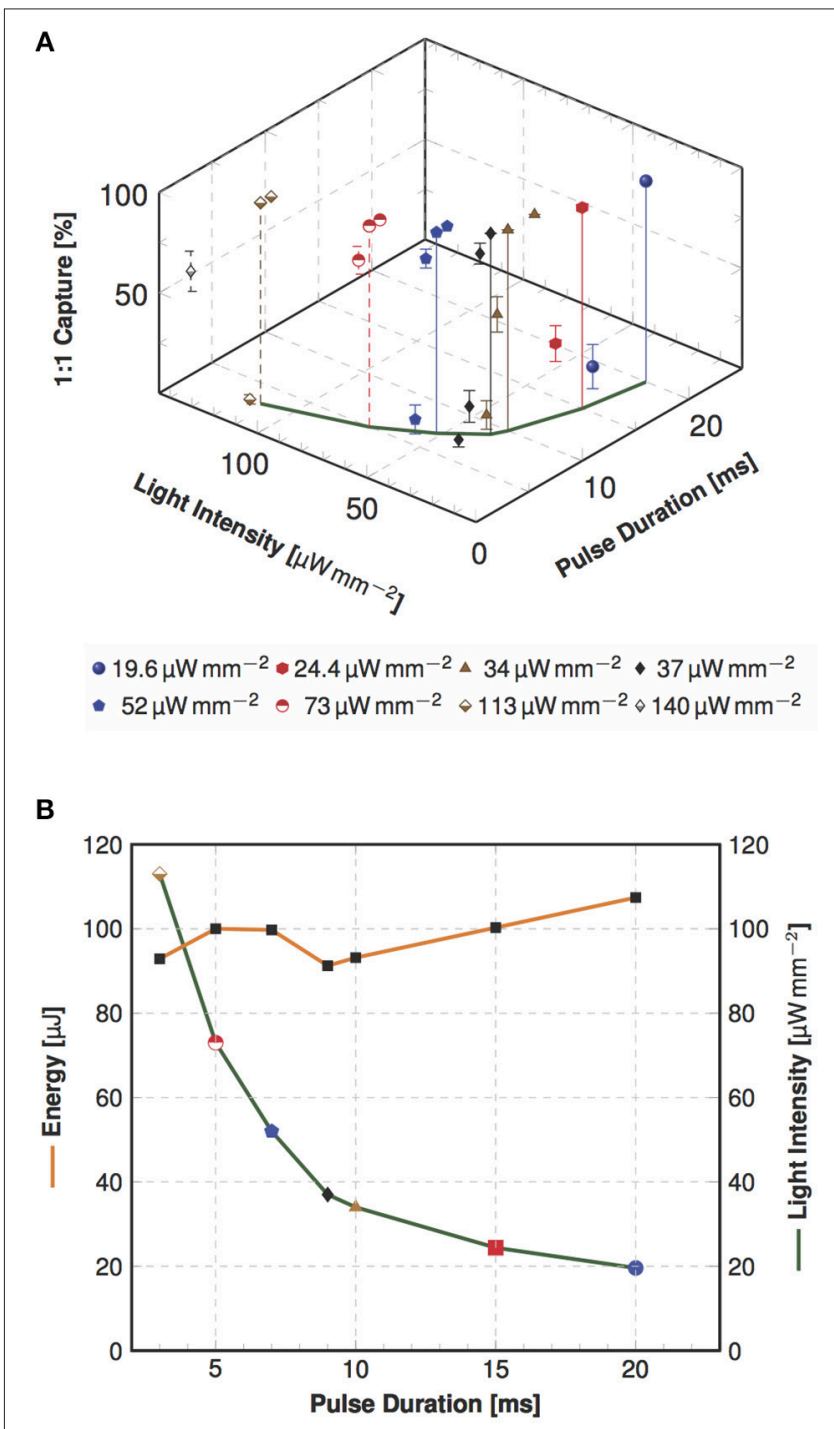

FIGURE 2 | Dependency of pulse duration and light intensity as well as pulse energy in global optogenetic pacing. (A) The three-dimensional plot illustrates the averaged success rate \pm SEM of 1:1 capture in dependency of

investigated combinations of light intensity and pulse duration (as indicated in Table 1). In this view the variation of measured capture rates is clearly visible, but still approving the dependency of successful 1:1 capture of light intensity and pulse duration. In the $x-y$ section are the most efficient combinations marked, which is visualized in a plain view in the next panel. (B) Shown are the most efficient combinations of required light intensity and energy for different pulse duration values $(N=6)$. The analysis shows a nearly constant energy, but an increase in intensity when the pulse duration is shortened.

light with optogenetic cardiac tissue can trigger excitation in single cardiomyocytes or united cell structures without activating the surrounding tissue structures or inducing critical Faraday reactions (Ambrosi and Entcheva, 2014). Here, we presented a setup to investigate arrhythmia termination using global illumination. The feasibility of photon initiated cardioversion was shown recently by Bruegmann et al. (2016), Crocini et al. (2016), and Nyns et al. (2016). Concentrating mainly

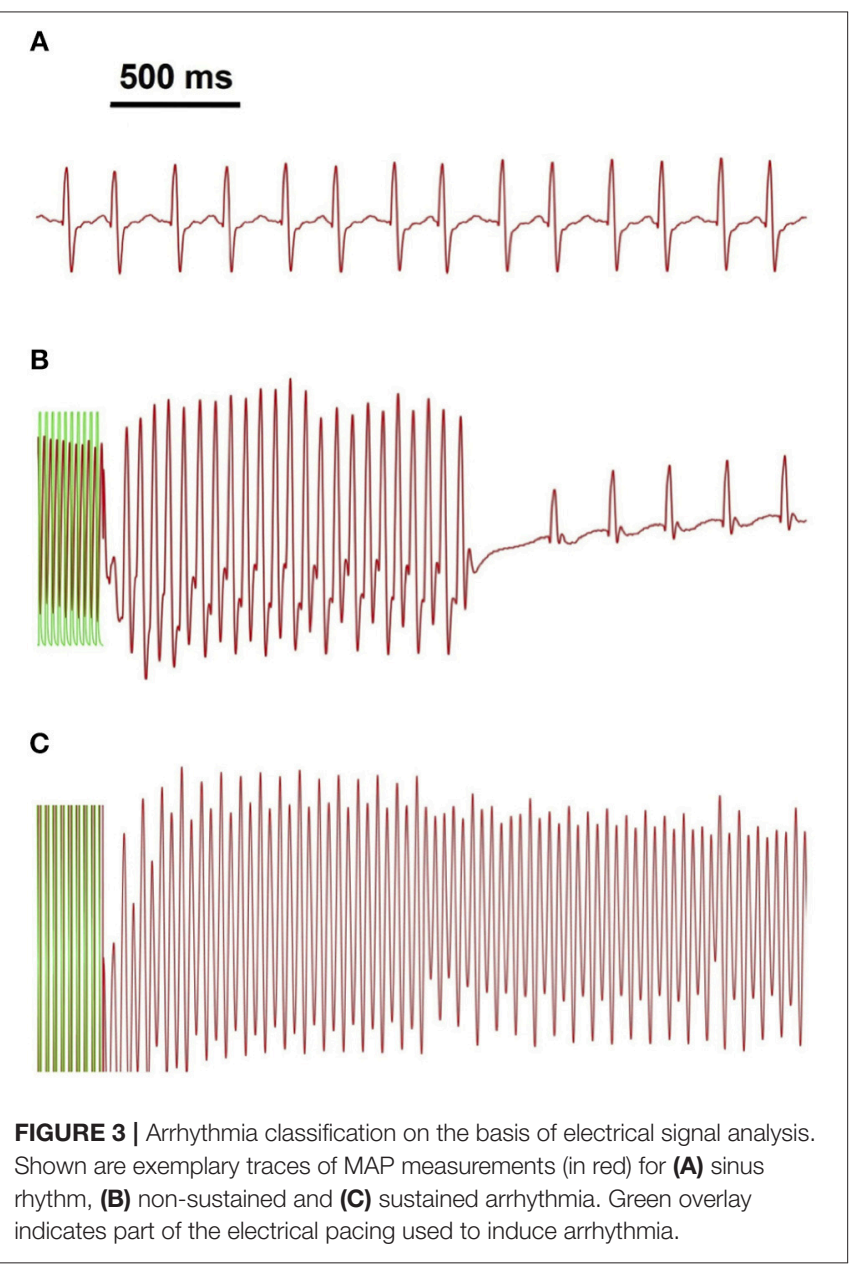

on low-numbered multi-site pacing Crocini et al. implemented a mechanistic approach using pulse series of an arrhythmiaspecific light pattern that enabled cardioversion using a total amount of $1.8 \mathrm{~mJ}$. In comparison the required light intensities up to $40 \mathrm{~mW} \mathrm{~mm} \mathrm{~mm}^{-2}$ exceeded the highest intensity implemented in our experiments by more than the 30 -fold. Furthermore, Bruegmann et al. described an illumination protocol applying $1 \%$ of the light intensity used by Crocini et al., but with a highly increased pulse duration of four pulses of $1 \mathrm{~s}$ with 1-5 s inbetween accounting for a total amount of energy of $228.8 \mathrm{~mJ}$. Having this in mind, in our experiments we concentrated on the characterization of the separate parameter combinations. In order to do so, the global stimulation of the whole cardiac tissue represents the maximum number of pacing sites available and thus it is comparable to conventional high-energy electrical defibrillation approaches. We showed the impact of illuminating the epicardial surface by pacing with the lowest intensities published so far. An interesting result is that the energy required to pace both globally and locally remained constant for each of the methods ( $98 \pm 5 \mu \mathrm{J}$ and $2.8 \pm 0.6 \mu \mathrm{J}$, respectively) and increased with a growing stimulation area. Certainly, the excitation origin depends on different factors such as wavelength or light propagation characteristics. An interesting observation 67 


\section{Global Optogenetic Stimulation to Terminate Arrhythmias}

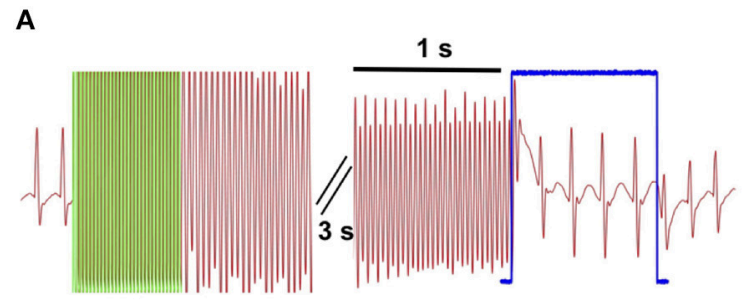

B
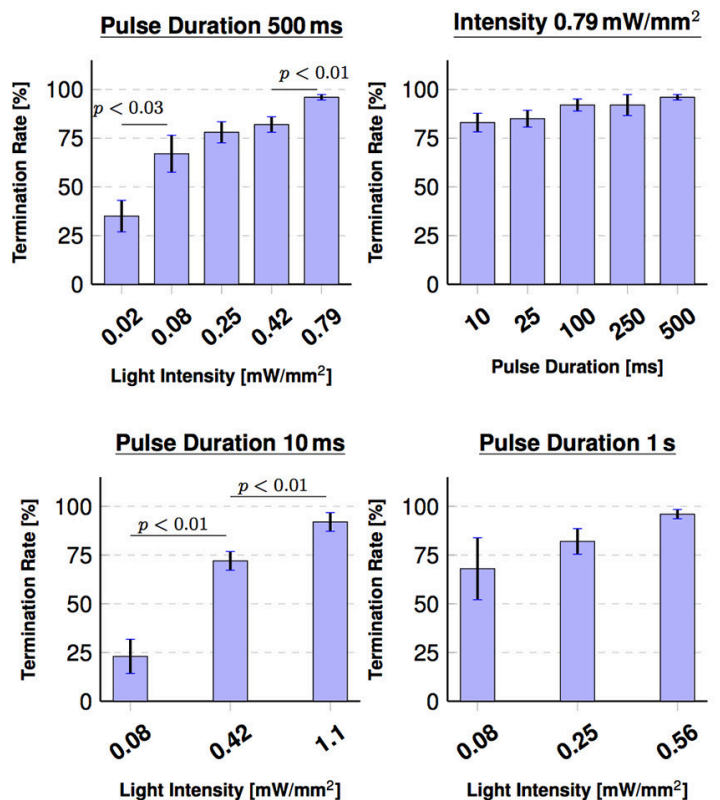

C

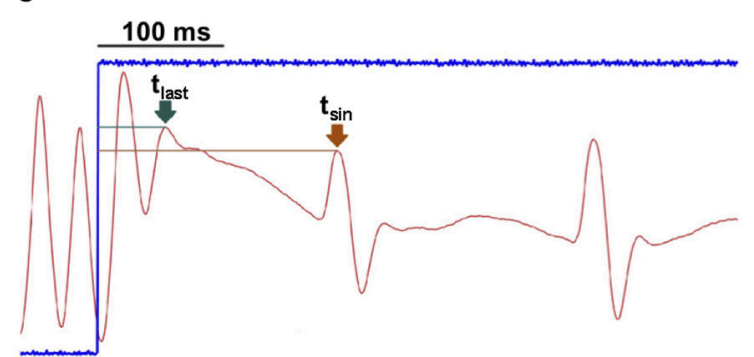

D
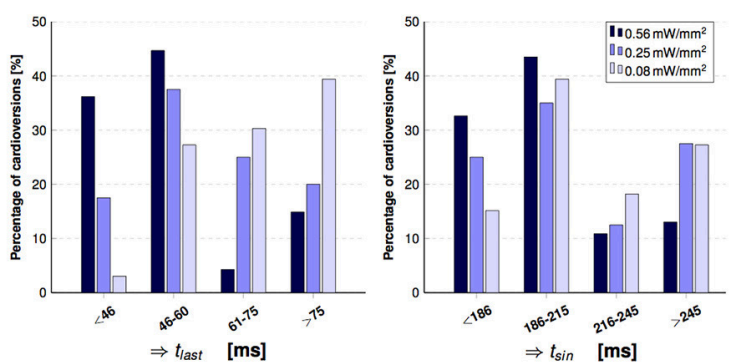

FIGURE 4 | Global illumination for cardioversion. (A) Shows the pseudoECG signal during arrhythmia evocation via electrical pacing and termination using global illumination (green overlay indicates electrical pacing at $50 \mathrm{~Hz}, 30$

(Continued)
FIGURE 4 | pulse). The arrhythmic conditions were terminated with a $1 \mathrm{~s}$ stimulation pulse (indicated in blue), during which the normal sinus rhythm already returned. (B) Summary of the influence of intensity and pulse duration on cardioversion attempts. Successful optogenetic defibrillation rates (percentage of successful attempts reported as mean $\pm \mathrm{SEM}, N=6$ ). (C) Visualizes the effect of the termination pulse shown in (A) in more detail. Two time points were defined in order to characterize the moment of arrhythmia termination for the $1 \mathrm{~s}$ pulses: $t_{\text {last }}$ (green arrow), which denotes the last peak of the arrhythmia and $t_{\sin }$ (brown arrow), which denotes the first peak of the sinus rhythm during optogenetic stimulation. (D) Charts indicating the effect of intensity on cardioversion times in percentage of arrhythmia terminated in those periods of $t_{\text {last }}$ (left) and $t_{\text {sin }}$ (right) for $0.56 \mathrm{~mW} \mathrm{~mm}^{-2}$ $(N=47), 0.25 \mathrm{~mW} \mathrm{~mm}^{-2}(\mathrm{~N}=40), 0.08 \mathrm{~mW} \mathrm{~mm}^{-2}(N=33)$.

during global photostimulation is the correlation between increasing light intensity and rising efficiency of cardioversion. Lilienkamp et al. introduced in silico results similar to our experimental findings, which can give hints that the overall size of an excitable medium has a direct effect on the lifetime of chaotic spatiotemporal dynamics, like the ones seen during arrhythmia (Lilienkamp et al., 2017). One possible explanation could be the change in penetration depth when using higher light intensities for photostimulation, hence depolarizing a greater number of cells and creating a thicker reversible conduction pattern. This was also described by Watanabe et al. in experiments with ventricular slices, where they showed a temporal decrease on the effective size available for the arrhythmia to wander (Watanabe et al., 2017). In their work, Watanabe et al. proved that by increasing the transmurality of illumination, the chances of terminating an anatomical re-entry on the slices increased. This effect could be lead back to the indispensable fact of reaching the critical cell number for excitation evocation (Zipes et al., 1975). In contrast, a varied pulse duration at constant light intensity might prolong the depolarization of the stimulated layers of cardiac tissue without effectively changing the size of the stimulated cardiac tissue (Bruegmann et al., 2010).

Optogenetic intensity-dependent effects have been recorded at the level of single cells, quasi-two dimensional cell cultures and organs (Bruegmann et al., 2010; Nussinovitch et al., 2014; Burton et al., 2015; Nussinovitch and Gepstein, 2015; Zaglia et al., 2015). Accordingly, the effect of light intensity as described by $t_{\text {last }}$ for the $1 \mathrm{~s}$ pulses could be explained by a theoretical combination of the amount of cells excited, the applied photocurrents and the conduction velocity of a generated excitation wave (Bruegmann et al., 2010; Burton et al., 2015; Zaglia et al., 2015). Moreover, the evidence showing that the majority of defibrillations took place during the onset of stimulation could be explained by the Channelrhodopsin-2 kinetics, since its photo-current reaches a peak during the first milliseconds of activation before dropping to a smaller current during continuous illumination (Nagel et al., 2003; Bruegmann et al., 2010). Yet, the difference on $t_{\text {sin }}$ for each intensity is not likewise obvious, since it could be more related to the intrinsic sinus period of the heart than to the light induced effect on cardioversion. On the other hand, the effect of the pulse duration, even when the difference seems not to be significant might lead to diverse 


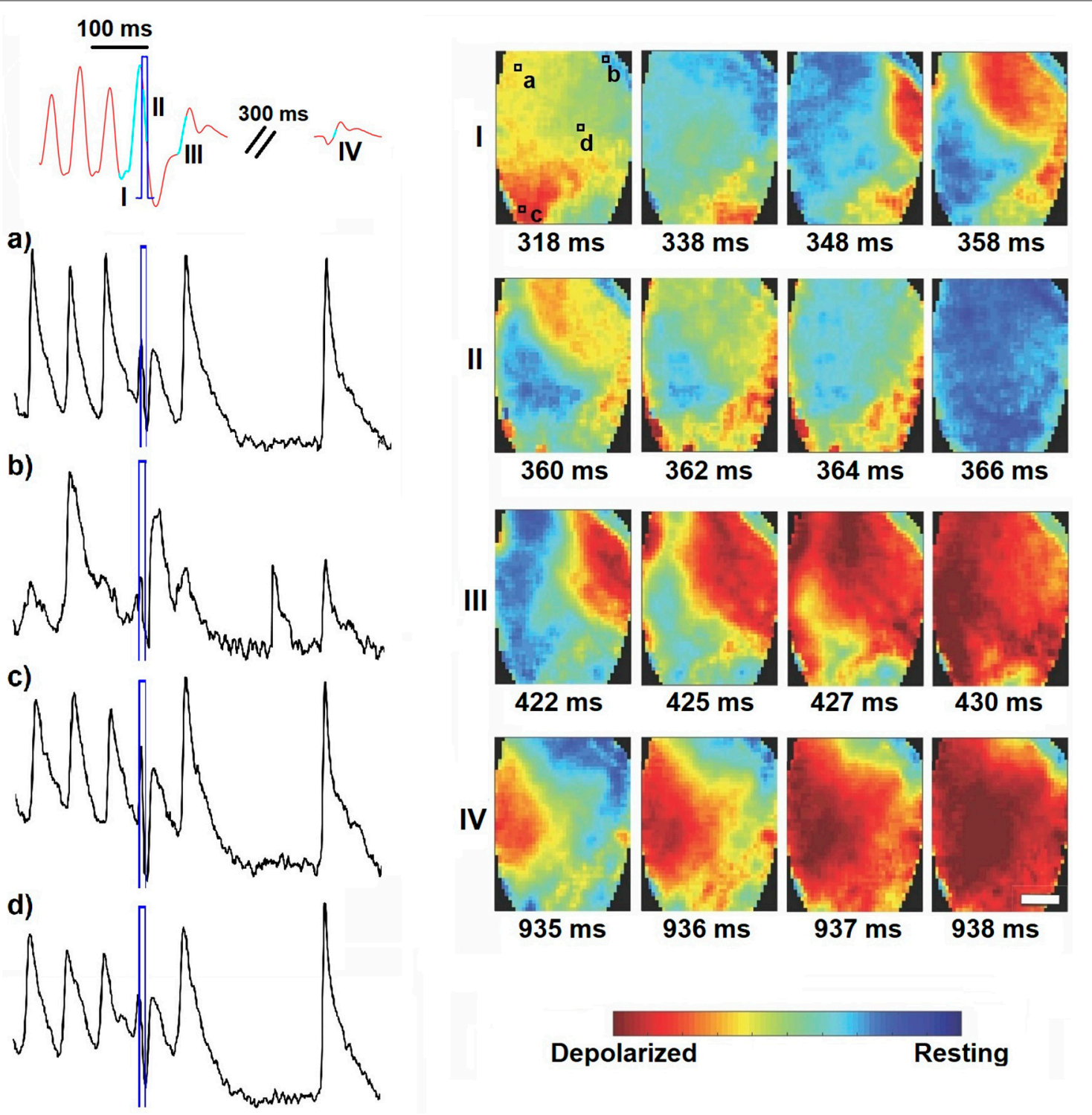

FIGURE 5 | Wave dynamics during short global light pulse (10 ms, $0.25 \mathrm{~mW} \mathrm{~mm}^{-2}$, highlighted in blue). Shown are the MAP recording (in red) and optical traces of several local spots on the ventricles (in black, as indicated in panel I). Optical mapping analysis revealed vortex-like electrical activity on the heart surface (image sequence I), which is disturbed by global illumination (image sequence II) and thus provoking wave collision resulting in annihilation (image sequence III). Sinus activity follows after a peak corresponding to depolarization of the whole heart (image sequence IV). The corresponding movie is available as (Supplementary Material Movie 2).

mechanisms of termination. Presumably, spiral disturbance is unlikely to happen during short pulses of 10 and $25 \mathrm{~ms}$. Nevertheless, a longer pulse duration such as $500 \mathrm{~ms}$ or $1 \mathrm{~s}$ can still disrupt the tachycardia even after the onset of the stimulus, leading to a higher success rate. According to the biophysics of ChR2, these light-activated channelrhodopsins are un-selective for different cations like $\mathrm{Ca}^{2+}$ or $\mathrm{Na}^{+}$(Schneider et al., 2015). Due to that fact, long photostimulation pulses probably lead to an accumulation of action potential relevant cations causing continuous depolarization to less negative membrane voltages. This assumption is supported by the fact that $10 \%$ of cardioversions for $1 \mathrm{~s}$ stimulus occurred during mid-late stimulation.

However, the results presented here strengthen the application of cardiac optogenetics, although some underlying mechanisms still remain to be part of ongoing research. Especially the investigation of defibrillation protocols consisting of multistage anti-fibrillation pacing as well as multi-site pacing strategies have to be addressed, since such experiments would help to understand the dependencies of phase-specific pacing and success rate of arrhythmia termination. Therefore, all ex vivo experiments and the basic classification of photostimulation 


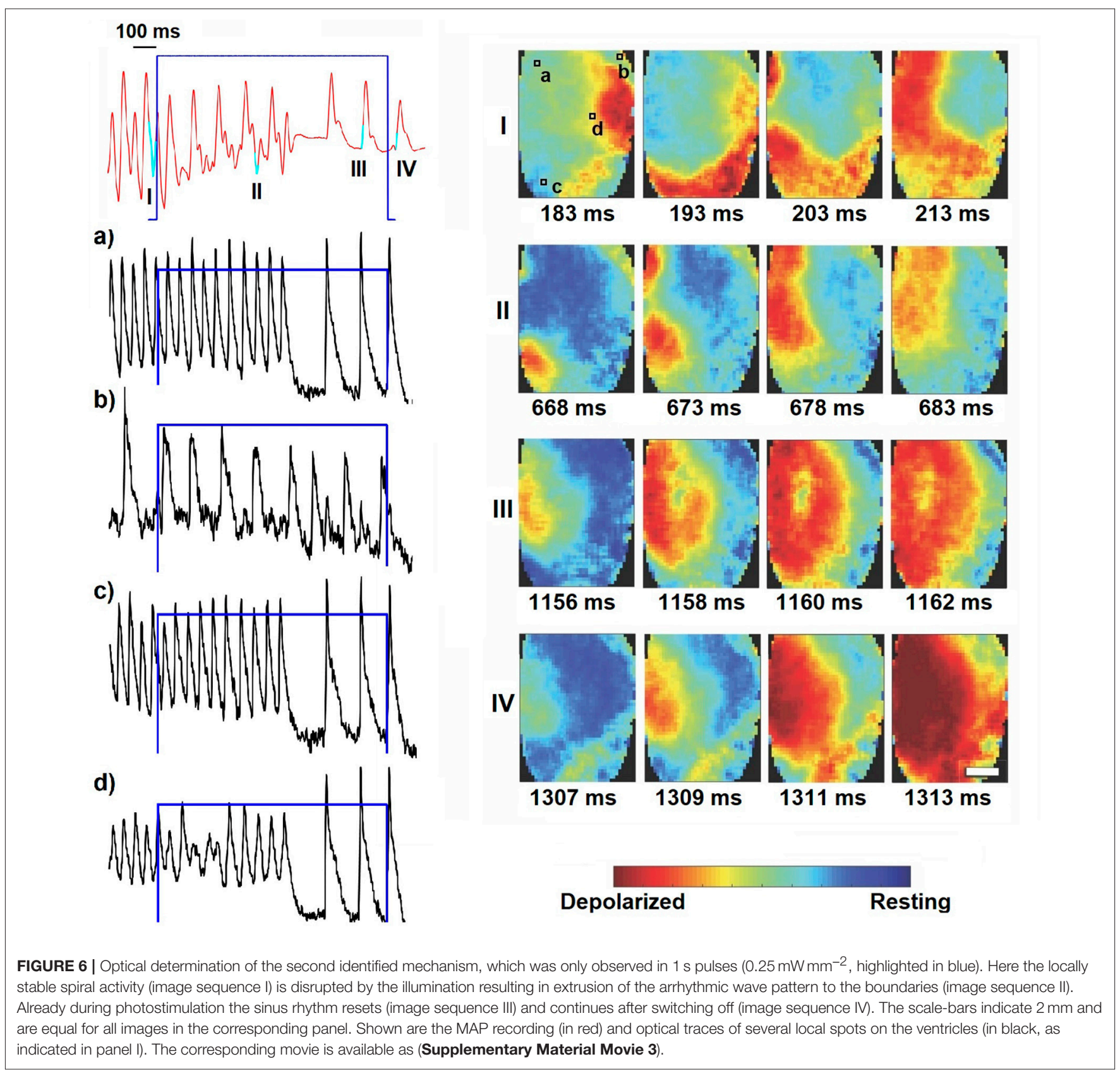

parameter function as a trigger for development, optimization and evaluation of a controllable as well as flexible light induced cardiac rhythm management. As a positive effect also the design of new non-electrical multi-site pacing methods with high potential of translation into clinically relevant approaches comes within reach.

\subsection{Limitations}

With the translation into in vivo conditions in mind, one has to mention that the ex vivo measurements lack the interference of blood specific absorption of ChR2 exciting wavelengths. Consequently, any effects during blue-light photostimulation in vivo would represent only a superficial excitation. Certainly, 70 the cardiac excitation conduction is not restricted to epicardial phenomena but also connected to complex transmural wave propagation (Christoph et al., 2018). Therefore, near infrared modulated opsins would support the translation of ex vivo experimental results into clinically relevant in vivo attempts (Karathanos et al., 2016). Here we successfully achieved cardioversion using single pulses of divers pulse duration by tuning light intensities between 0.56 and $1.1 \mathrm{mWmm}^{-2}$, delivering total energies $3-153.6 \mathrm{~mJ}$ to the epicardium. The importance of this low-intensity, pulse duration versatility relies on the fast spatiotemporal dynamics of arrhythmia, where the optimal duration of the stimulus could be arrhythmia-specific dependent. That would also result in a smaller amount of light 


\subsection{Results (publication)}

being delivered to the cardiomyocytes located transmurally. The more precise estimation of excited cells within the ventricular wall and the characterization of light propagation through cardiac tissue are subject of current research projects. In the presented study we only examined murine hearts, for which reason the presented parameter findings can solely be considered as species specific. For larger species with much thicker ventricular walls or fatty epicardial regions the light intensity actually impacting light-sensitive cardiomyocytes will differ significantly. However, the gotten insights and general conclusions, like the fact that illuminating the whole epicardial surface will result in a decrease of minimum light intensity should be accountable also for larger species.

Furthermore, induced photochemical reactions during illumination are possible sources of direct cell response (Lubart et al., 2007). Lubart et al. showed that especially visible light $(400 \mathrm{~nm}$ to $700 \mathrm{~nm})$ could stimulate photobiomodulation and photosensitization in cardiomyocytes. These processes are inter alia initiated by generation of reactive oxygen species, which in return can lead to a change of the redox state of the cardiac cell and thus have indirect influence on the calcium induced calcium release (CICR) via redox sensitive L-type calcium channels. Although, compared to the energy used by Lubart et al. with $3.6 \mathrm{~J} \mathrm{~cm}^{-2}$ the total energy applied for global illumination in this study was $0.01-0.61 \mathrm{Jcm}^{-2}$, which may have alleviated photochemical effects. Hence, the measurement and characterization of potential photobiomodulation with regard to repeated rapid photostimulation are considered for ongoing projects.

Experiments including optical mapping were done with Blebbistatin, which reduces motion artifacts due to its electromechanical uncoupling function. Since we used stimulation light of a blue wavelength $(470 \mathrm{~nm})$ it is important to mention, that the uncoupling effects of Blebbistatin could be reversed by blue light illumination (Fedorov et al., 2012). In our experiments we did not observe such light induced effects on the motion inhibition, which could be related to the fact that Fedorov et al. used much shorter wavelengths for illumination than we applied. This observation was also published by Swift et al. (2012). Nevertheless, it should be emphasized that Blebbistatin does possibly alter the metabolic state of ischemic cardiomyocytes (Swift et al., 2012).

\section{CONCLUSION}

According to recent cardiac optogenetic studies the usage of photodefibrillation seems to be feasible (Bruegmann et al.,

\section{REFERENCES}

Ambrosi, C., and Entcheva, E. (2014). Optogenetics' promise: pacing and cardioversion by light? Future Cardiol. 10, 1-4. doi: 10.2217/fca.13.89

Bruegmann, T., Boyle, P. M., Vogt, C. C., Karathanos, T. V., Arevalo, H. J., Fleischmann, B. K., et al. (2016). Optogenetic defibrillation terminates ventricular arrhythmia in mouse hearts and human simulations. J. Clin. Invest. 126, 3894-3904. doi: 10.1172/JCI88950
2016, Crocini et al., 2016; Richter et al., 2016). Here we investigated for the first time the effects of global optogenetic epicardial illumination which benefits from the larger area covered to decrease the intensity required to stimulate the heart. In consequence, we managed to terminate arrhythmic excitation patterns using pulse lengths spanning three orders of magnitude, demonstrating an efficient and versatile low-intensity and low-energy method to investigate arrhythmia dynamics and manipulation. Besides, we observed two different mechanisms leading to optogenetic cardioversion, which exhibit not the same behavior than electrical cardioversion.

\section{AUTHOR CONTRIBUTIONS}

RQ performed the experiments and analyzed the data. RQ and CR designed research, experiments and wrote the paper. SL and LD-M contributed to the discussion and edited the manuscript. All authors read and approved the manuscript.

\section{FUNDING}

This project has received funding from the European Union's Horizon 2020 Programme through the project Advanced BiomEdical OPTICAL Imaging and Data Analysis (BEOPTICAL) under grant agreement number 675512. The research leading to these results received funding by the European Community's Seventh Framework Programme FP7/2007-2013 under grant agreement number HEALTH-F2-2009-241526. Additional support was provided by the DZHK e.V., the German Federal Ministry of Education and Research (BMBF, project FKZ 031A147, Go-Bio), the German Research Foundation (DFG, Collaborative Research Centers SFB 1002, Projects B05 and C03 and SFB 937, Project A18) and the Max Planck Society.

\section{ACKNOWLEDGMENTS}

We want to thank Marion Kunze and Tina Althaus for their excellent technical assistance during experimental setup and experiment conduction. Many thanks to Thomas Lilienkamp and Ulrich Parlitz for fruitful discussions and their thoughtprovoking impulses.

\section{SUPPLEMENTARY MATERIAL}

The Supplementary Material for this article can be found online at: https://www.frontiersin.org/articles/10.3389/fphys. 2018.01651/full\#supplementary-material

Bruegmann, T., Malan, D., Hesse, M., Beiert, T., Fuegemann, C., Fleischmann, B., et al. (2011). Channelrhodopsin2 expression in cardiomyocytes: a new tool for light-induced depolarization with high spatio-temporal resolution in vitro and in vivo. Thorac. Cardiovasc. Surg. 59:MO19. doi: 10.1055/s-0030-12 69109

Bruegmann, T., Malan, D., Hesse, M., Beiert, T., Fuegemann, C. J., Fleischmann, B. K., et al. (2010). Optogenetic control of heart muscle in vitro and in vivo. Nat. Methods 7, 897-900. doi: 10.1038/nmeth.1512 


\section{Global Optogenetic Stimulation to Terminate Arrhythmias}

Burton, R. A. B., Klimas, A., Ambrosi, C. M., Tomek, J., Corbett, A., Entcheva, E., et al. (2015). Optical control of excitation waves in cardiac tissue. Nat. Photonics 9, 813-816. doi: 10.1038/nphoton.2015.196

Christoph, J., Chebbok, M., Richter, C., Schrder-Schetelig, J., Bittihn, P., Stein, S., et al. (2018). Electromechanical vortex filaments during cardiac fibrillation. Nature 555:667 EP. doi: 10.1038/nature26001

Christoph, J., Schröder-Schetelig, J., and Luther, S. (2017). Electromechanica optical mapping. Prog. Biophys. Mol. Biol. 130, 150-169. doi: 10.1016/j.pbiomolbio.2017.09.015

Crocini, C., Ferrantini, C., Coppini, R., Scardigli, M., Yan, P., Loew, L. M., et al. (2016). Optogenetics design of mechanistically-based stimulation patterns for cardiac defibrillation. Sci. Rep. 6:35628EP. doi: 10.1038/srep35628

Davidenko, J., Pertsov, A., Salamonsz, R., Baxter, W., and Jalif, J. (1992). Stationary and drifting spiral waves of excitation in isolated cardiac muscle. Nature 355 349-351. doi: 10.1038/355349a0

Deisseroth, K. (2011). Optogenetics. Nat. Methods 8, 26-29. doi: 10.1038/nmeth.f.324

Diaz-Maue, L., Luther, S., and Richter, C. (2018). "Towards optogenetic control of spatiotemporal cardiac dynamics," in Proceedings SPIE-Optogenetics and Optical Manipulation 2018 (San Francisco, CA: SPIE), 1482G.

Efimov, I. R., Aguel, F., Cheng, Y., Wollenzier, B., and Trayanova, N (2000). Virtual electrode polarization in the far field: implications for external defibrillation. Am. J. Physiol. Heart Circ. 279, H1055-H1070. doi: 10.1152/ajpheart.2000.279.3.H1055

Exner, D. (2005). Is antitachycardia pacing a safe and efficacious alternative to shocks for fast ventricular tachyarrhythmia treatment? Nat. Clin. Pract. Cardiovasc. Med. 2:68. doi: 10.1038/ncpcardio0116

Fedorov, V., Lozinsky, I., Sosunov, E., Anyukhovsky, E., Rosen, M., Balke, C., et al (2012). Application of blebbistatin as an excitation-contraction uncoupler for electrophysiologic study of rat and rabbit hearts. Heart Rhythm. 4, 619-626. doi: 10.1016/j.hrthm.2006.12.047

Fenton, F., Luther, S., Cherry, E., Otani, N., Krinsky, V., Pumir, A., et al. (2009). Termination of atrial fibrillation using pulsed low-energy far-field stimulation. Circulation 120, 467-476. doi: 10.1161/CIRCULATIONAHA.108.825091

Glukhov, A. V., Flagg, T. P., Fedorov, V. V., Igor, R. E., and Nichols, C. G. (2010) Differential katp channel pharmacology in intact mouse heart. J. Mol. Cell. Cardiol. 48, 152-160. doi: 10.1016/j.yjmcc.2009.08.026

Jalifé, J., Gray, R., Morley, G., and Davidenko, J. (1998). Self-organization and the dynamical nature of ventricular fibrillation. Chaos 8, 79-93. doi: 10.1063/1.166289

Janardhan, A., Li, W., Fedorov, V., Yeung, M., Wallendorf, M., Schuessler R., et al. (2012). A novel low-energy electrotherapy that terminates ventricular tachycardia with lower energy than a biphasic shock when antitachycardia pacing fails. JACC 60, 2393-2398. doi: 10.1016/j.jacc.2012.0 8.1001

Karathanos, T., Bayer, J., Wang, D., Boyle, P., and Trayanova, N. (2016). Opsin spectral sensitivity determines the effectiveness of optogenetic termination of ventricular fibrillation in the human heart: a simulation study. J. Physiol. 594, 6879-6891. doi: 10.1113/JP271739

Killeen, M., Thomas, G., Gurung, I., Goddard, C., Fraser, J., MahautSmith, M., et al. (2007). Arrhythmogenic mechanisms in the isolated perfused hypokalaemic murine heart. Acta Physiol. 189, 33-46. doi: 10.1111/j.1748-1716.2006.01643.x

Lilienkamp, T., Christoph, J., and Parlitz, U. (2017). Features of chaotic transients in excitable media governed by spiral and scroll waves. Phys. Rev. Lett. 119, 1-5. doi: 10.1103/PhysRevLett.119.054101

Lubart, R., Lavi, R., Friedmann, H., and Rochkind, S. (2007). Photochemistry and photobiology of light absorption by living cells. Photomed. Laser Surg. 24 179-185. doi: 10.1089/pho.2006.24.179

Luther, S., Fenton, F. H., Kornreich, B. G., Squires, A., Bittihn, P., Hornung, D., et al. (2011). Low-energy control of electrical turbulence in the heart. Nature 475, 235-239. doi: 10.1038/nature10216

Nagel, G., Szellas, T., Huhn, W., Kateriya, S., Adeishvili, N., Berthold, P., et al. (2003). Channelrhodopsin-2, a directly light-gated cation-selective membrane channel. Proc. Natl. Acad. Sci. U.S.A. 100, 13940-13945. doi: 10.1073/pnas.1936192100
Nussinovitch, U., and Gepstein, L. (2015). Optogenetics for in vivo cardiac pacing and resynchronization therapies. Nat. Biotechnol. 33, 750-754. doi: $10.1038 /$ nbt.3268

Nussinovitch, U., Shinnawi, R., and Gepstein, L. (2014). Modulation of cardiac tissue electrophysiological properties with light-sensitive proteins. Cardiovasc. Res. 102, 176-187. doi: 10.1093/cvr/cvu037

Nyns, E. C., Kip, A., Bart, C. I., Plomp, J. J., Zeppenfeld, K., Schalij, M. J., et al. (2016). Optogenetic termination of ventricular arrhythmias in the whole heart: towards biological cardiac rhythm management. Eur. Heart J. 38:ehw574. doi: 10.1093/eurheartj/ehw574

Panfilov, A., and Holden, A. (1990). Self-generation of turbulent vortices in a two-dimensional model of cardiac tissue. Phys. Lett. A 151, 23-26. doi: 10.1016/0375-9601(90)90840-K

Pumir, A., and Krinsky, V. (1999). Unpinning of a rotating wave in cardiac muscle by an electric field. J. Theoret. Biol. 199, 311-319. doi: 10.1006/jtbi.1999.0957

Pumir, A., Nikolsky, V., Hoerning, M., Isomura, A., Agladze, K., Yoshikawa, K., et al. (2007). Wave emission from heterogeneities opens a way to controlling chaos in the heart. Phys. Rev. Lett. 99:208101. doi: 10.1103/PhysRevLett.99.208101

Richter, C., Christoph, J., Lehnart, S. E., and Luther, S. (2016). “Optogenetic light crafting tools for the control of cardiac arrhythmias," in Optogenetics - Methods and Protocols, Vol. 1408, ed A. Kianianmomeni (New York, NY: Springer Science+Business Media), 293-302.

Schneider, F., Grimm, C., and Hegemann, P. (2015). Biophysics of channelrhodopsin. Annu. Rev. Biophys. 44, 167-86. doi: 10.1146/annurev-biophys-060414-034014

Swift, L., Asfour, H., Posnack, N., Arutunyan, A., Kay, M., and Sarvazyan, N. (2012). Properties of blebbistatin for cardiac optical mapping and other imaging applications. Pflugers Arch. Eur. J. Physlol. 464, 503-512. doi: 10.1007/s00424-012-1147-2

Takagi, S., Pumir, A., Pazó, D., Efimov, I., Nikolski, V., and Krinsky, V. (2004). Unpinning and removal of rotating wave in cardiac muscle. Phys. Rev. Lett. 93:058101. doi: 10.1103/PhysRevLett.93.058101

Watanabe, M., Feola, I., Majumder, R., Jangsangthong, W., Teplenin, A. S., Ypey, D. L., et al. (2017). Optogenetic manipulation of anatomical re-entry by lightguided generation of a reversible local conduction block. Cardiovasc. Res. 113, 354-366. doi: 10.1093/cvr/cvx003

Wathen, M. S., DeGroot, P. J., Sweeney, M. O., Stark, A. J., Otterness, M. F., Adkisson, W. O., et al. (2004). Prospective randomized multicenter trial of empirical antitachycardia pacing versus shocks for spontaneous rapid ventricular tachycardia in patients with implantable cardioverter-defibrillators: Pacing fast ventricular tachycardia reduces shock therapies. Circulation 110, 2591-2596. doi: 10.1161/01.CIR.0000145610.64014.E4

Wilde, A. (1994). K+atp channel opening and arrhythmogenesis. J. Cardiovasc. Pharmacol. 24(Suppl. 4), S35-S40.

Zaglia, T., Pianca, N., Borile, G., Da Broi, F., Richter, C., Campione, M., et al. (2015). Optogenetic determination of the myocardial requirements for extrasystoles by cell type-specific targeting of ChannelRhodopsin-2. Proc. Natl. Acad. Sci. U.S.A. 112:1509380112. doi: 10.1073/pnas.1509380112

Zipes, D. P., Fischer, J., King, R. M., Nicoll, A. d., and Jolly, W. W. (1975). Termination of ventricular fibrillation in dogs by depolarizing a critical amount of myocardium. Am. J. Cardiol. 36, 37-44. doi: 10.1016/0002-9149(75)90865-6 Zipes, D. P., and Jalife, J. (2009). Cardiac Electrophysiology: From Cell to Bedside. Philadelphia, PA: Saunders; Elsevier.

Conflict of Interest Statement: The authors declare that the research was conducted in the absence of any commercial or financial relationships that could be construed as a potential conflict of interest.

Copyright (C) 2018 Quiñonez Uribe, Luther, Diaz-Maue and Richter. This is an openaccess article distributed under the terms of the Creative Commons Attribution License (CC BY). The use, distribution or reproduction in other forums is permitted, provided the original author(s) and the copyright owner(s) are credited and that the original publication in this journal is cited, in accordance with accepted academic practice. No use, distribution or reproduction is permitted which does not comply with these terms. 


\subsection{Discussion \& Conclusions}

The use of global illumination to terminate arrhythmias did result in a drop in intensity or pulse duration required compared to previous optogenetic defibrillation approaches [12, 19]. Naturally, the drawback of this method is that illuminating the whole epicardial surface could lead to technical challenges. However, if global illumination is achievable, we have shown here that it offers versatility between the duration of the pulse and intensity applied without the need of high intensities.

We also took an extra step into the mechanistics behind optogenetic cardioversion and classified termination into either annihilation or perturbation, depending on the time it took before the arrhythmia signal disappeared. This classification can still have room for improvement, but what was clearly evident is that the majority of terminations take place within the first $100 \mathrm{~ms}$. Therefore, we dedicated Chapter 9 to analyze a larger number of arrhythmia termination times, since it could give a hint for optimizing the stimuli and the processes of optogenetic arrhythmia termination.

Combining optical mapping with optogenetic stimulation allowed us to image the differences of annihilation and perturbation [63]. However, there are still some difficulties found in order to obtain a clear signal from the dye while spontaneously optogenetically stimulating the heart. Therefore, for this work further processing of the optical mapping data was required, since the voltage dye signal is shifted due to the blue light and in the case of more smooth analysis or investigation of smaller voltage changes on the epicardium this could become a greater limitation. In addition, as mentioned in Chapter 6 sections 6.2 .1 and 6.2.2, blebbistatin has an effect on both light attenuation and in the stimulation threshold of ChR2 mice hearts. In the same line, blebbistatin has been shown to affect conductivity in cell cultures [83], decrease susceptibility of cardiac arrhythmia in mice hearts [4] and affect induction of fibrillation in rabbit hearts [10]. Considering all the points mentioned before, it is of high importance that any optogenetic experiment performed using optical mapping and blebbistatin consider potential effects on the illumination thresholds and other electrophysiological measurements and results.

Now, that there is a better understanding on how arrhythmias behave under global illumination, I envision two ways of moving forward in cardiac optogenetic research. 1) A step into structured illumination would provide more insights into the control of arrhythmias using light. Even though there have been different studies employing specific illumination patterns and its capabilities [19, 50, 15, 77], the field has still to exploit the spatio-temporal advantages of optogenetics in order to explore initiation, behavior and termination of cardiac arrhythmia and cardiac electrophysiology in general [27, 69, 97]. Nevertheless, 2) it is important to also consider larger animal models. As described during the introduction and also tested in the light absorption experiments, ventricular thickness and light attenuation blood and other structures of the heart will play a major role in the efficiency of optogenetic arrhythmia treatment. Testing how larger light-sensitive hearts respond to methods such as global, local and structured 
illumination will have a critical impact on the future of optogenetics as a potential therapy.

In conclusion, we designed an experimental setup capable of panoramic stimulation of ChR2 transgenic mice heart. With this we showed that global illumination requires shorter pulses and smaller intensities to efficiently terminate arrhythmias. Furthermore, we inspected the temporal behavior in which optogenetics leads to cardioversion. Either by annihilation, taking place early or perturbation, taking longer time. 


\section{Optogenetic cardioversion based on arrhythmia cycle length and optogenetic cardioversion based on morphology}

\subsection{Introduction and Aim}

Arrhythmias can be classified based on the morphology of the electrical signal recorded, with a monomorphic signal showing a repetitive pattern and a polymorphic showing temporal changes in their morphology or frequency (Figure 8.1). The repetitive pattern in a monomorphic signal suggests a stable origin of tachycardia from a focus or structural substrate for example generated from the re-entrant activity of a stable spiral in one of the ventricles. A polymorphic signal has a continually changing signal which suggests a varying ventricular activation sequence for example generated by one or even more drifting spirals [21, 37, 75].
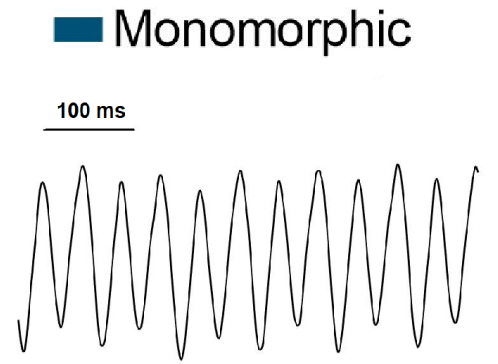

- Polymorphic

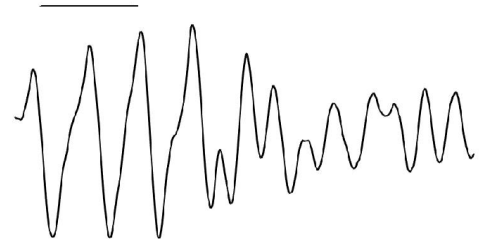

Figure 8.1.: Monomorphic and polymorphic ventricular tachycardia in the mouse heart. Monophasic action potential signals obtained from contact electrodes of ChR2mice Langendorff-perfused mice hearts. On the left an example of a monomorphic VT and on the right polymorphic VT. Pinacidil was used to induce arrhythmia as reported in the Materials and Methods section. Bars represent $100 \mathrm{~ms}$.

Different studies using electrical cardioversion have shown that the energy required to terminate an arrhythmia depends on the morphology, with monomorphic arrhythmias re- 
vealing lower thresholds compared to the more complex polymorphic events [93, 42]. Even though in optogenetics this effect has not been thoroughly investigated, Nyns and colleagues revealed that termination of ventricular arrhythmia using single $470 \mathrm{~nm}$ light pulses of 1 second in rat hearts transduced with the red-activatable channelrhodopsin (ReaChR) terminated $97 \%$ of monomorphic arrhythmias compared to $57 \%$ of the polymorphic cases [61].

Since different arrhythmia types have also been observed in mice [53, 84], my next step was to investigate the effect of the arrhythmia morphology on the efficacy of optogenetic cardioversion. The second part of the results of this chapter is devoted to the investigation of the arrhythmia morphology on the success of the termination attempt.

The first part of this Chapter focuses on the impact of the illumination time based on arrhythmia cycle length. In order to investigate this, the concept of $t_{\text {last }}$ which was defined in Chapter 7 as the time it takes to terminate an arrhythmia from the beginning of stimulation until the last peak of the arrhythmia. In the same Chapter it was shown that $86 \%$ of the arrhythmias terminated with a single $1 \mathrm{~s}$ pulse using the global stimulation setup exhibited $\leq$ 2 arrhythmia cycles before being terminated, a term we called arrhythmia annihilation [67]. Considering this I wanted to explore whether the efficiency of arrhythmia termination could depend on the number of arrhythmia cycles/periods the heart is illuminated.

\subsection{Results}

\subsubsection{Illumination time based on arrhythmia cycle length}

As mentioned before, $86 \%$ of all the successful cardioversions using $1000 \mathrm{~ms}$ took place within 2 arrhythmia cycles from the beginning of stimulation. Therefore, I hypothesized that a global stimulation lasting 2 arrhythmia periods should be sufficient to return the heart rhythm into its sinus state. In order to investigate the influence of the illumination duration based on arrhythmia periods on the cardioversion success rate, I opted to test the efficiency of pulses lasting $5 \mathrm{~ms}, 40 \mathrm{~ms}, 80 \mathrm{~ms}$ and $250 \mathrm{~ms}$ which corresponds to approximately $0,1,2$ and 6 arrhythmia periods as the mean arrhythmia frequency is $f_{\text {arr }}=24 \pm 5 \mathrm{~Hz}$ (period $\sim 40 \mathrm{~ms}$ ). A representative example of each of the pulses tested is shown in Figure 8.2, where as anticipated the $5 \mathrm{~ms}$ pulse covers less than an arrhythmia period, the $40 \mathrm{~ms}$ illuminates for one period, $80 \mathrm{~ms}$ around two cycles and $250 \mathrm{~ms}$ will be covering more cycles.

Figure 8.3 A plots the results obtained for the analyzed pulses lengths. Interestingly, both $40 \mathrm{~ms}$ and $250 \mathrm{~ms}$ showed the highest efficiency at $71 \%$, while $5 \mathrm{~ms}$ pulses showed the only significant drop in efficiency and terminated only $30 \%$ of the tachycardia induced. Since our main reference to choose the pulse lengths was based on the chances of an arrhythmia being terminated within 2.5 arrhythmia cycles or less, we also analyzed this event for each 
A

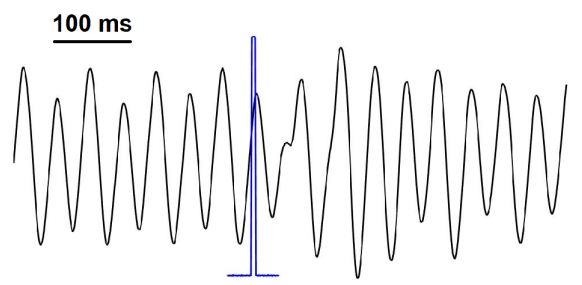

C

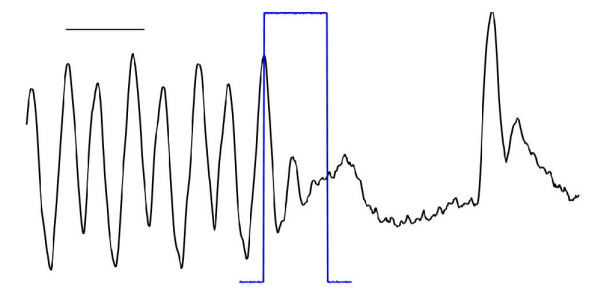

B

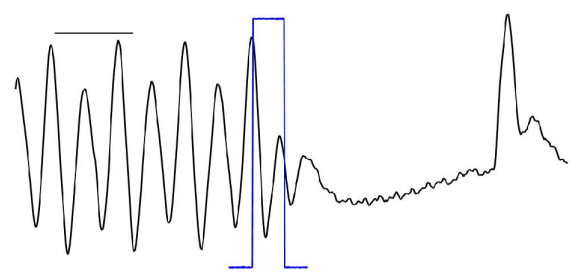

D

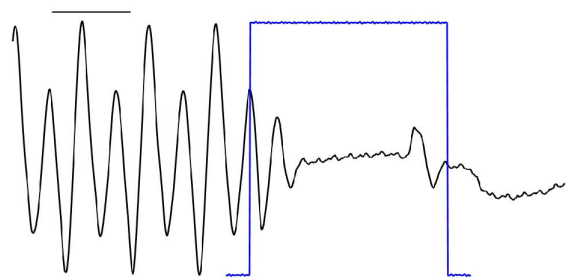

Figure 8.2.: Optogenetic cardioversion based on arrhythmia cycles. Examples of pulse widths of (A) $5 \mathrm{~ms}$, (B) $40 \mathrm{~ms}$, (C) $80 \mathrm{~ms}$ and (D) $250 \mathrm{~ms}$. Black represents ECG signal and blue pulse stimulation, black line marks $100 \mathrm{~ms}$.

case. All the pulses tested, independent of the width, terminated at least half of the arrhythmia in this manner, while more than $70 \%$ of the cases of the $40 \mathrm{~ms}$ and $80 \mathrm{~ms}$ took place in this range (Figure $8.3 \mathrm{~B}$ ).
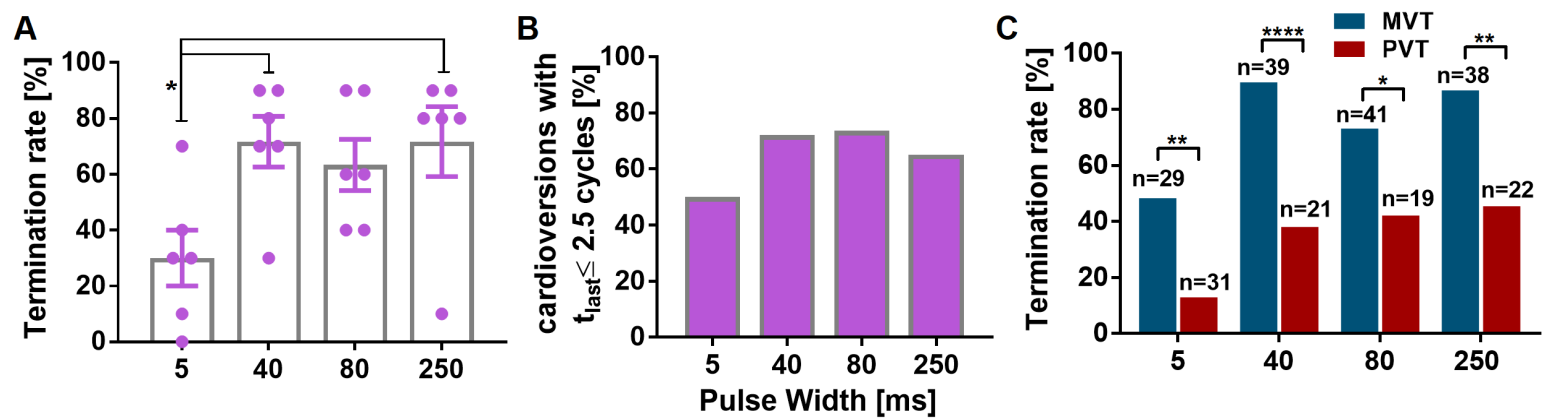

Figure 8.3.: Arrhythmia cycles termination rate. (A) The efficiency for 5, 40, 80 and $250 \mathrm{~ms}$ pulses. Reported as mean \pm s.e.m., $N=6$ mice, each data point represents one heart with $n=10$ attempts. Performed Dunn's multiple comparisons test $(* \mathrm{p}<0.05)$. (B) Percentage $(\%)$ of terminated arrhythmias within 2.5 arrhythmia cycles from the beginning of stimulation. (C) Cardioversion success rate grouped by morphology. Monomorphic ventricular tachycardia (MVT) in blue, polymorphic ventricular tachycardia (PVT) in red. Fisher's exact test performed for comparison $(* \mathrm{p}<0.05, * * \mathrm{p}<0.01, * * * * \mathrm{p}<0.0001)$. 


\subsubsection{Optogenetic Cardioversion of Monomorphic and Polymorphic Ventricular Tachycardia}

\section{Termination based on cycle length with constant intensity}

As a next step to our inspection on the effect of the arrhythmia cycles used as pulse length on Global Optogenetic Cardioversion (G.O.C.), we analyzed the efficiency of this technique to terminate arrhythmia based on morphology. Accordingly, all the arrhythmia from the four explored pulse lengths $(n=240)$ were classified into either monomorphic (Figure 8.1A) or polymorphic (Figure 8.1 B) tachycardia, revealing that $61 \%$ of the arrhythmia belonged to the first group. Also, G.O.C. at this light intensity was evidently more efficient terminating monomorphic than polymorphic arrhythmia (76\% vs $32 \%$ success rates).

Furthermore, by evaluating morphology dependence for each pulse length separately an significant increased capability to terminate monomorphic arrhythmias was observed in all the cases Figure 8.2 C, with observed similar rates when illuminating for $40 \mathrm{~ms}, 80 \mathrm{~ms}$ and $250 \mathrm{~ms}$. This again demonstrated that efficiency for the three pulses greater than the arrhythmia period was alike.

\section{Effect of intensity on mono- and polymorphic VT termination}

Since there was no difference found in the termination of mono- and poly- morphic arrhythmias with the pulse lengths tested, I opted to investigate using greater differences, such as $10 \mathrm{~ms}, 100 \mathrm{~ms}$ and $1000 \mathrm{~ms}$, and at the same time vary the intensity for each of the pulses. In a similar manner, arrhythmias from previously performed experiments were classified and group before determining the termination efficiencies.

The results are illustrated in Figure 8.4, which is composed of two rows. The top row displays the termination success rates of ventricular tachycardia using three different light intensities of $1000 \mathrm{~ms}, 100 \mathrm{~ms}$ and $10 \mathrm{~ms}$ for illumination intensities of $0.56 \mathrm{~mW} / \mathrm{mm}^{2}$, $0.25 \mathrm{~mW} / \mathrm{mm}^{2}$ and $0.08 \mathrm{~mW} / \mathrm{mm}^{2}$. The bottom row displays the efficiency of termination divided by morphology of arrhythmia (mono- or polymorphic ventricular tachycardia) of the same experiments. Classification of the arrhythmias resulted in $60-80 \%$ of the cases as monomorphic arrhythmia, depending on the subgroup of intensity/pulse-width observed. Also, regardless of the subgroup, polymorphic events were less frequent for all the groups analyzed.

The longest pulse width analyzed was 1 second. For this case the termination efficiency of the tested intensities was higher for monomorphic arrhythmia, with a decrease in the difference for higher intensities (Figure 8.4 A, bottom). Moreover, none of the intensities terminated 

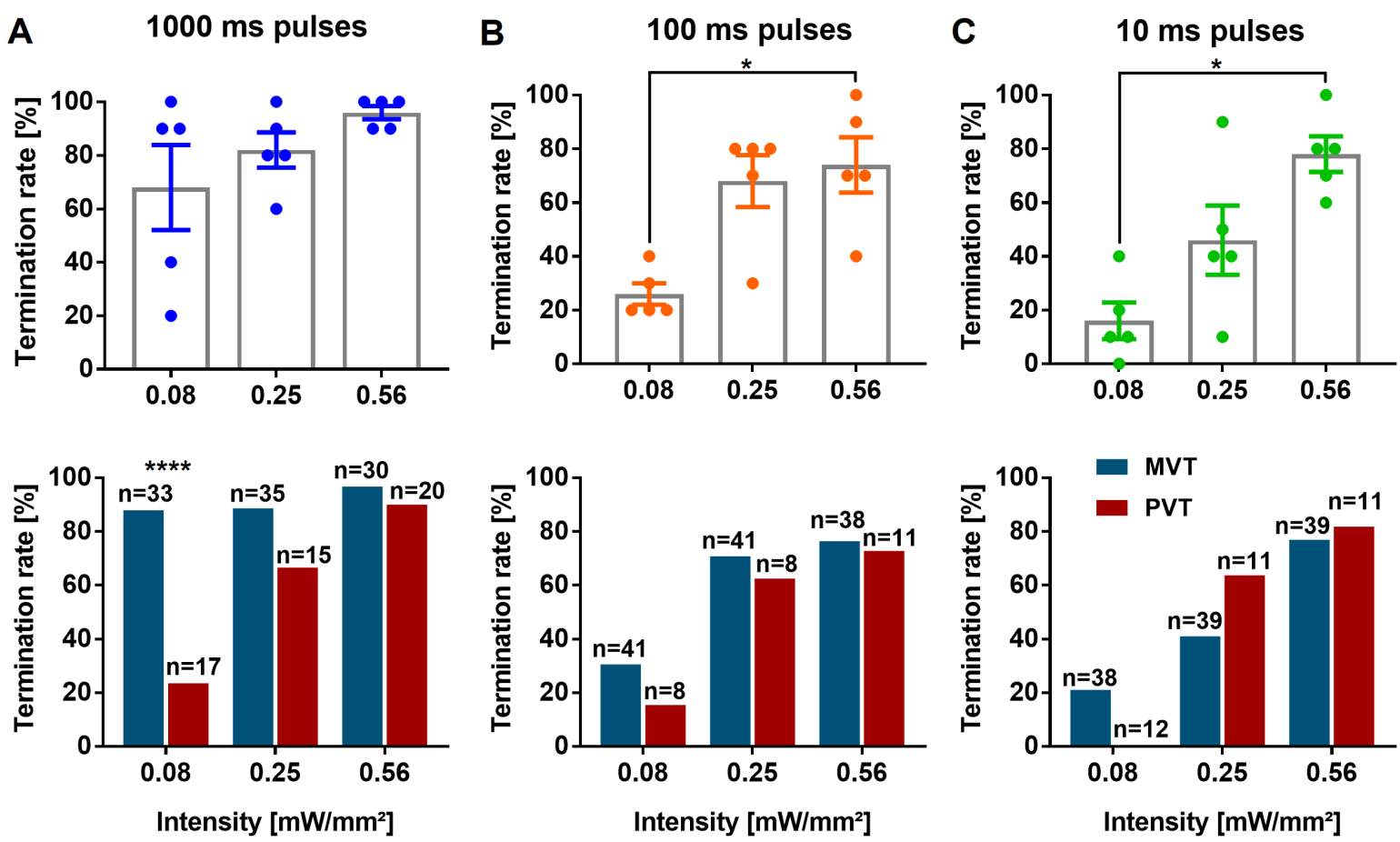

Figure 8.4.: Optogenetic termination rate of monomorphic and polymorphic arrhythmia. The top row consists of bar plots displaying the general arrhythmia termination efficiency during global stimulation for single pulses of (A)1000 ms, (B) $100 \mathrm{~ms}$ and $(\mathbf{C}) 10 \mathrm{~ms}$ shown as mean \pm s.e.m. with each dot representing a single heart with $n=10$ termination attempts per intensity (total $n=50$ ). The bottom row displays the results of the same experiments divided for success rate of monomorphic (VT) and polymorphic (PVT) ventricular tachycardias of each combination of pulse width and intensity. The n-number indicates the total number of arrhythmias observed for each group. For arrhythmia termination performed one-way ANOVA $(* \mathrm{p}<0.05)$. For morphology comparison performed Fisher's exact test $(* * * * \mathrm{p}<0.0001)$

less than $85 \%$ of monomorphic events and for the lowest intensity of $0.08 \mathrm{~mW} / \mathrm{mm}^{2}$ the termination efficiency for monomorphic arrhythmia was $88 \%$ compared to $25 \%$ in polymorphic.

Decreasing the pulse width to $100 \mathrm{~ms}$ resulted in a less evident difference in efficiencies 8.4 B), where again the termination success was higher for the less complex arrhythmias and the most clear case was in the lowest intensity with $30 \%$ of MVT terminated compared with a drop to $15 \%$ of PVT. Lastly, using $10 \mathrm{~ms}$ flashes resulted in higher cardioversion efficiency for polymorphic arrhythmias for the highest intensities but in a similar fashion, for the lowest intensity it was an evident difference with monomorphic arrhythmia being terminated $16 \%$ of the times compared to $0 \%$ success in the case of polymorphic arrhythmias 8.4 C). However, for both the $100 \mathrm{~ms}$ and $10 \mathrm{~ms}$ cases is difficult to draw any conclusions since the number of PVT was lower. 
In summary, using the lowest intensity of $0.08 \mathrm{~mW} / \mathrm{mm}^{2}$ for any pulse lengths resulted in termination rates which are systematically larger for arrhythmias with a monomorphic ECG pattern compared to arrhythmias with a polymorphic pattern. Moreover, at this intensity only the pulses with duration of $1 \mathrm{~s}$ were able to terminate efficiently at $88 \%$ compared to the shorter pulses which both terminated less than one third of the attempts. For the middle intensity of $0.25 \mathrm{~mW} / \mathrm{mm}^{2}$ the efficiency is greater for monomorphic arrhythmia using $1 \mathrm{~s}$ pulses, but decreases for $100 \mathrm{~ms}$ long pulses and changing to a higher termination of polymorphic arrhythmia for the shortest $10 \mathrm{~ms}$ pulses. For the highest intensity of $0.56 \mathrm{~mW} / \mathrm{mm}^{2}$ there was no clear difference of termination for any morphology in any of the three pulse widths tested. I conclude that 1) longer pulses facilitate monomorphic arrhythmia termination, 2) when using low intensities, there is higher chance of monomorphic arrhythmia termination than polymorphic arrhythmia termination and 3) for higher intensities there are no significant differences in termination rates. As previously mentioned, it is difficult to obtain a representative statistical analysis for some cases since the number of polymorphic VT was considerable lower.

\subsection{Discussion \& Conclusions}

At first, we investigated the role of the pulse lengths based on tachyarrhythmia cycles in global optogenetic cardioversion. Our experiments revealed that for the intensity tested, the three pulses that were at least as long as an arrhythmia period showed comparable rates of cardioversion, independent of the morphology of the arrhythmia. In a similar manner, our previous experiments [67] (Chapter 7) using $0.79 \mathrm{~mW} / \mathrm{mm}^{2}$ showed a drop only from $95 \%$ to $85 \%$ when decreasing the pulse width from $500 \mathrm{~ms}$ to $25 \mathrm{~ms}$. Results from the same experiments suggest that the differences in success between pulse widths becomes broader for smaller intensities, explaining the evident contrast between the $5 \mathrm{~ms}$ long attempts and the rest of the widths compared. For the experiments performed under these conditions a pulse of at least one cycle length increase the success substantially. However, further experiments using other intensities and pulses are needed in order to strengthen this theory.

Interestingly, for both the shortest pulse of $5 \mathrm{~ms}$ and the longest pulse of $250 \mathrm{~ms}$, cardioversion took place after 2.5 cycles in more than $50 \%$ of the cases. This offers a first hint of a possible common mechanism behind global optogenetic cardvioversion. One of the drawbacks of counting the cycles is that arrhythmias can show different frequencies and polymorphic arrhythmias might not have a single frequency, therefore a better way to investigate termination is by looking into how long does a pulse of light takes to terminate an arrhythmia. Chapter 9 is focused on analyzing this specific period of time, the role of intensity and pulse length on this task and the possibility of a common mechanism behind this specific span of time.

The first grouping of monomorphic and polymorphic arrhythmia for the arrhythmia cycles with an intensity of $0.25 \mathrm{~mW} / \mathrm{mm}^{2}$ showed higher success rates for monomorphic tachy- 
cardia, for all the pulse lengths tested. As well, varying the intensity for the $1 \mathrm{~s}$ stimuli evidenced the same behavior. However, shortening the pulses made this conclusion uncertain. A theory could be that the termination of monomorphic arrhythmias is both intensity and pulse width dependent, in a way that a longer pulse can compensate for a low intensity, and that the termination of polymorphic arrhythmias depends mainly on the intensity of the stimulus.

One of the limitations of the morphology experiments is that the number of polymorphic events can vary per heart and in most of the cases it is lower than the number of monomorphic arrhythmia. Therefore, this analysis could benefit from a larger number of experiments, or larger number of cardioversion attempts per heart. Moreover, morphology classification was performed visually, which could lead to error in certain cases. Considering there is a wide spectrum of arrhythmia morphologies, it can be challenging to develop a tool for automatic classification but consistency in classification would be increased.

Both of the analysis performed in this chapter -arrhythmia cycle and morphology dependencycan potentially lead to interesting and revealing results. However, they both need further experiments that can help us better understand the roles played by the intensity and the pulse width, since for the results varied once these parameters were changed. 



\section{Cardioversion times}

\subsection{Introduction and Aim}

In the previous chapters I have mainly analyzed the effects of light intensity and pulse width on the success rate of global optogenetic cardioversion. And in Chapter 7 the term $t_{\text {last }}$ was defined as the time it takes from the beginning of optogenetic stimulation until the last peak of the arrhythmia (Fig. 9.1 A, B), in other words how long it takes an optogenetic stimulus to terminate the arrhythmia, and naturally it can only be measured for successful attempts [67].

Due to the nature of electrical shocks, this parameter could not be determined or measured. However, it can be observed and characterized in the electrophysiological recordings of hearts submitted to optogenetic arrhythmia termination. In our publication [67] we already performed the first analysis on it, and we observed that in optogenetic cardioversions using pulses of $1000 \mathrm{~ms}$, an increase in light intensity leads to decrease in $t_{\text {last }}$.

A deeper analysis of $t_{\text {last }}$ could lead to a better understanding of optogenetic cardioversion and simultaneously of cardiac arrhythmia dynamics and behavior. Therefore, I dedicated this chapter to describe $t_{\text {last }}$ from experiments performed previously, as well as to interrogate factors affecting the time it takes to optogenetically terminate an arrhythmia using global illumination.

\subsection{Results}

As a first step, I decided to perform the analysis of $t_{\text {last }}$ first from a global or common perspective, where I pooled together all the cardioversions from different intensities and pulse widths. Later, I investigated the differences in cardioversion times depending on specific widths and intensities of the stimuli, as well as the morphology of the arrhythmia. 
A

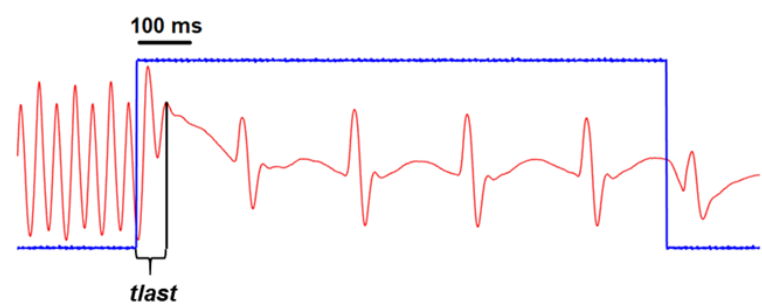

B

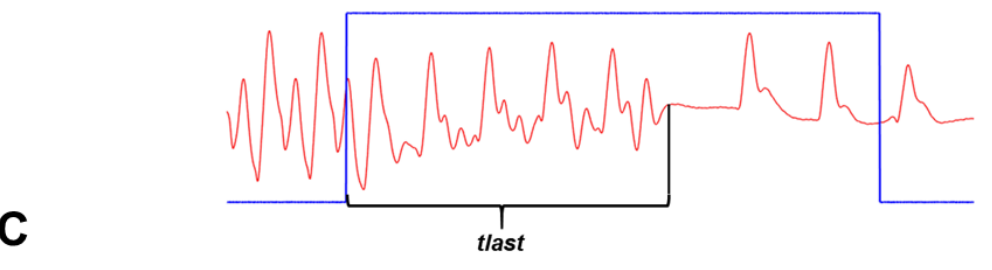

C

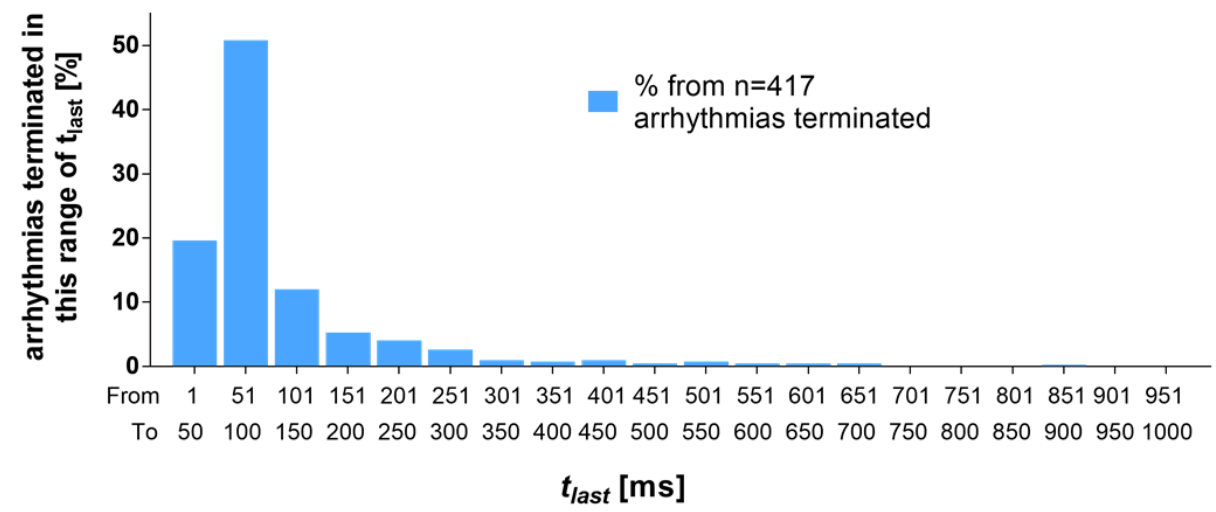

Figure 9.1.: Examples of $\boldsymbol{t}_{\text {last }}$. Monophasic action potential (MAP) signals showing examples of $t_{\text {last }}$, defined as the time it takes to terminate an arrhythmia from the moment an optogenetic stimulus starts. In (A) the tachycardia disappears within the first $100 \mathrm{~ms}$, on the onset of stimulation. While in (B) the tachycardia lasts beyond $100 \mathrm{~ms}$ and shows activity while the heart is being illuminated. Black lines denote $100 \mathrm{~ms}$, MAP signals are shown in red and global illumination in blue. (C) Histogram showing the percentage of arrhythmias terminated within a range of $t_{\text {last }}$ in groups of $50 \mathrm{~ms}$, starting from $1 \mathrm{~ms}$ to $50 \mathrm{~ms}$. $(n=417)$ successful arrhythmia terminations.

\subsubsection{Arrhythmia termination takes place in a specific range}

In order to have an overview of the range of cardioversion times I examined this feature for different experiments. $t_{\text {last }}$ from all the experiments used in Chapter $8(n=417)$ were grouped and plotted in a histogram. Since for the optogenetic cardioversion experiments we considered a termination attempt as successful when it terminated the arrhythmia within $1 \mathrm{~s}$ from the beginning of stimulation, the histogram obtained consists of bins of $50 \mathrm{~ms}$, with the firs one considering the range of $1 \mathrm{~ms}$ to $50 \mathrm{~ms}$, and the last bin the range of $951 \mathrm{~ms}$ to $1000 \mathrm{~ms}$ 
(Figure 9.1 C ). Ventricular arrhythmias induced in our experiments present frequencies in he range $15 \mathrm{~Hz}$ to $30 \mathrm{~Hz}$, with an average of $24 \pm 5 \mathrm{~Hz}$ (mean \pm S.D.).

$88 \%$ of all successful cardioversions took place within $t_{\text {last }} \leq 200 \mathrm{~ms}$, and more specifically half of all terminations took place in the range between $51 \mathrm{~ms}$ to $100 \mathrm{~ms}$. Moreover (not visible in Fig. 9.1 C), only 2 events $(0.5 \%)$ were shorter than $25 \mathrm{~ms}$. On the other hand, just $2.4 \%$ of the events took place on the second half of the spectrum, between $501 \mathrm{~ms}$ to $1000 \mathrm{~ms}$.

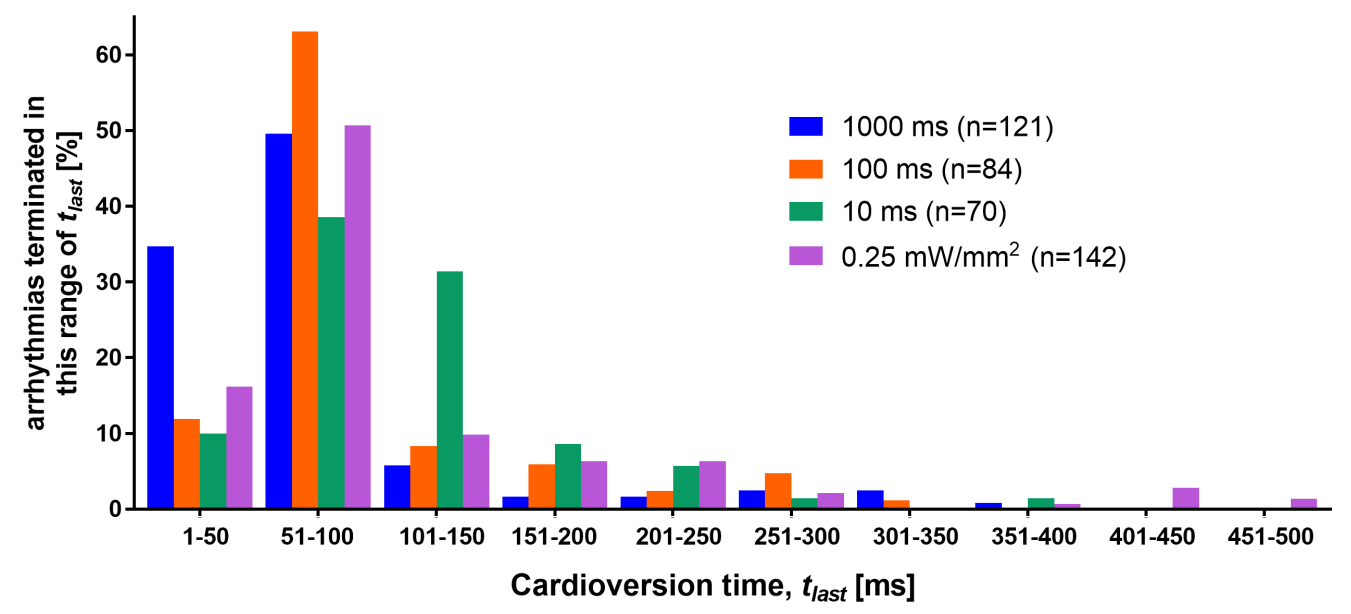

Figure 9.2.: Cardioversion times $\left(t_{\text {last }}\right)$ divided per group. Grouped histograms showing the percentage of arrhythmias terminated within a range of $t_{\text {last }}$ in groups of $50 \mathrm{~ms}$, starting from $1 \mathrm{~ms}$ to $50 \mathrm{~ms}$. From all successful cardioversions $(n=417)$, $t_{\text {last }}$ were grouped. Cardioversions experiments using pulses of $1000 \mathrm{~ms}$ (blue), $100 \mathrm{~ms}$ (orange), $10 \mathrm{~ms}$ (green) were applied using intensities of $0.08 \mathrm{~mW} / \mathrm{mm}^{2}$, $0.25 \mathrm{~mW} / \mathrm{mm}^{2}$ and $0.56 \mathrm{~mW} / \mathrm{mm}^{2}$ and the cardioversion experiments performed with an intensity of $0.25 \mathrm{~mW} / \mathrm{mm}^{2}$ were performed using pulse widths of $5 \mathrm{~ms}$, $40 \mathrm{~ms}, 80 \mathrm{~ms}$ and $250 \mathrm{~ms}$ (Figs. $8.3 \& 8.4$ ).

Since these results obtained come from arrhythmias being terminated in different groups of experiments, I also divided the data into groups following the original experiments performed for Chapter 8 (Figures 8.3 A and 8.4 A, B and C) in order to check for similar behaviors. The groups are: $n=121$ from pulses of $1000 \mathrm{~ms}, n=84$ from pulses of $100 \mathrm{~ms}$, $n=70$ from pulses of $10 \mathrm{~ms}$, with the three groups with varying intensities of $0.08 \mathrm{~mW} / \mathrm{mm}^{2}$, $0.25 \mathrm{~mW} / \mathrm{mm}^{2}$ and $0.56 \mathrm{~mW} / \mathrm{mm}^{2}$. And the last group of $n=142$ with pulses of constant intensity of $0.25 \mathrm{~mW} / \mathrm{mm}^{2}$ and varying pulse widths of $5 \mathrm{~ms}, 40 \mathrm{~ms}, 80 \mathrm{~ms}$ and $250 \mathrm{~ms}$. Figure 9.2 provides a histogram with the events for each group, this histogram only shows the range from $1 \mathrm{~ms}$ to $500 \mathrm{~ms}(97.6 \%$ of the data) but the percentages are still based on the range from $1 \mathrm{~ms}$ to $1000 \mathrm{~ms}$.

Consistent with the collective behavior, all groups showed the highest amount of terminations taking place between $51 \mathrm{~ms}$ to $100 \mathrm{~ms}$ and a $t_{\text {last }} \leq 200 \mathrm{~ms}$ in at least $80 \%$ of the cases, with zero to one case below $25 \mathrm{~ms}$. Moreover, $84 \%$ of the cases were $\leq 100 \mathrm{~ms}$ for 
the $1000 \mathrm{~ms}$, compared to $48 \%$ for the $10 \mathrm{~ms}$ group. In any case, this group terminated arrhythmias in $\leq 200 \mathrm{~ms}$ for $88 \%$ of the cases. After analyzing $t_{\text {last }}$ by groups of experiments an analysis of the intensities and pulse widths is detailed on the following section.

\subsubsection{Influence of illumination intensity and duration on the cardioversion time}

As a next step I also examined the effect of the intensity and pulse width on $t_{\text {last }}$. In order to do so, the cardioversions performed using pulses of $10 \mathrm{~ms}, 100 \mathrm{~ms}$ and $1000 \mathrm{~ms}$ were further divided depending on the intensities applied $\left(0.08 \mathrm{~mW} / \mathrm{mm}^{2}, 0.25 \mathrm{~mW} / \mathrm{mm}^{2}\right.$ and $0.56 \mathrm{~mW} / \mathrm{mm}^{2}$ ), obtaining a total of 9 different groups. Considering that the distribution of cardioversion times has a long tail that could lead to problems when applying a statistical test, I decided to remove the values that corresponded to the largest $10 \%$ of each group before obtaining the mean values of $t_{\text {last }}$ for each group, plotted in Figure $9.3 \mathrm{~A}$.

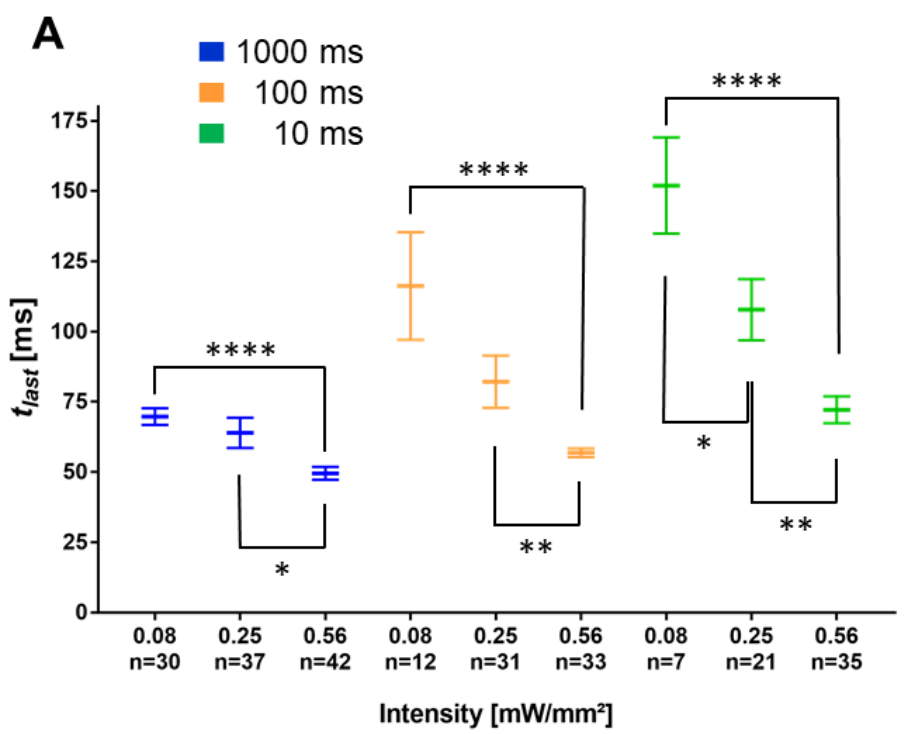

B

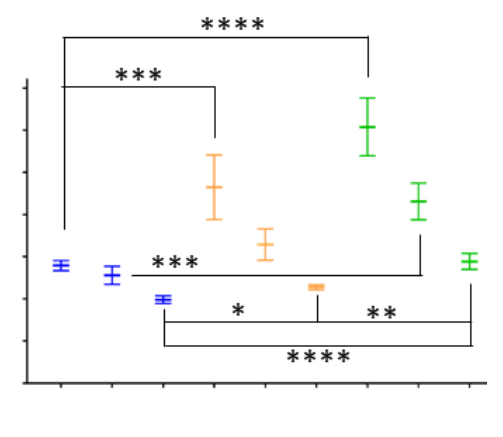

Figure 9.3.: Effect of light intensity and pulse width on $\boldsymbol{t}_{\text {last }}$. (A) Mean values of $t_{\text {last }}$ for pulses of $1000 \mathrm{~ms}$ (blue), $100 \mathrm{~ms}$ (orange), $10 \mathrm{~ms}$ (green) and varying intensities of $0.08 \mathrm{~mW} / \mathrm{mm}^{2}, 0.25 \mathrm{~mW} / \mathrm{mm}^{2}$ and $0.56 \mathrm{~mW} / \mathrm{mm}^{2}$ after removing the $10 \%$ largest values. t-test summaries performed for differences between intensities within the same pulse width. (B) Summaries of performed t-tests to the mean $t_{\text {last }}$ for constant intensity and varying pulse widths. Unpaired t-tests performed for comparisons $(* \mathrm{p}<0.05, * * \mathrm{p}<0.01, * * * \mathrm{p}<0.001, * * * * \mathrm{p}<0.0001)$.

Regardless of the pulse width, an increase in intensity led to a decrease in $t_{\text {last }}$. Furthermore, keeping the intensity constant and decreasing the pulse width also leads to an increase in mean $t_{\text {last }}$. In consequence, stimulations with the longest pulses and greatest intensities managed to terminate arrhythmias at a faster pace. Combining these results with the results of 
the termination success rates, it can be concluded that optogenetic cardioversion with higher efficiency also require less time to terminate arrhythmias.

As a last comparison, I aimed at understanding how the morphology of the terminated arrhythmia could affect the cardiversion time. Figure 9.4 displays the mean values of $t_{\text {last }}$ for terminated monomorphic and polymorphic arrhythmia (Figure 8.1). Cardioversions in monomorphic arrhythmia took in average $99.2 \pm 5.7 \mathrm{~ms}$ (mean \pm S.D.) compared to $135.2 \pm$ $14.4 \mathrm{~ms}$ when the terminated arrhythmia presented a polymorphic signal.

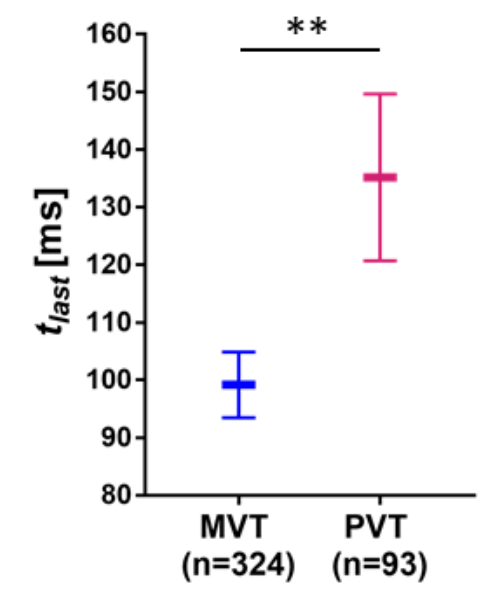

Morphology of Terminated Arrhythmia (Mono- or Polymorphic Ventricular Tachycardia)

Figure 9.4.: $\boldsymbol{t}_{\text {last }}$ of Monomorphic (MVT) and Polymorphic Ventricular Tachycardia (PVT). Mean values of $t_{\text {last }}$ for all the arhythmias grouped by morphology. Blue for MVT $(n=324)$, pink for PVT $(n=93)$. Unpaired t-test performed for comparison $(* * \mathrm{p}<0.01)$.

\subsubsection{Arrhythmic wave alteration after stimulation}

Using the optical mapping technique, I compared the electrical recording from the surface electrodes with the optical signals obtained during optogenetic arrhythmia termination as a manner to complement my investigation of the mechanisms behind optogenetic cardioversion and $t_{\text {last }}$. Blebbistatin was used to halt the mechanical beating of the heart without stopping its electrical activity and track using the voltage membrane dye Di-4-ANBDQPQ as described in Chapter 5 and in [67]. In general, optogenetic cardioversion experiments were performed without application of blebbistatin. At the end of an experiment and only if the heart was still viable I would record optical mapping measurements.

Since the main characteristic of $t_{\text {last }}$ is that the majority $(\sim 90 \%)$ of cardioversions take place within $200 \mathrm{~ms}$ from the beginning of stimulation, I aimed to investigate recordings with 
this feature. In total 9 recordings from 5 different hearts were analyzed. Figures 9.5 and 9.6 are representative of the events identified using optical mapping.

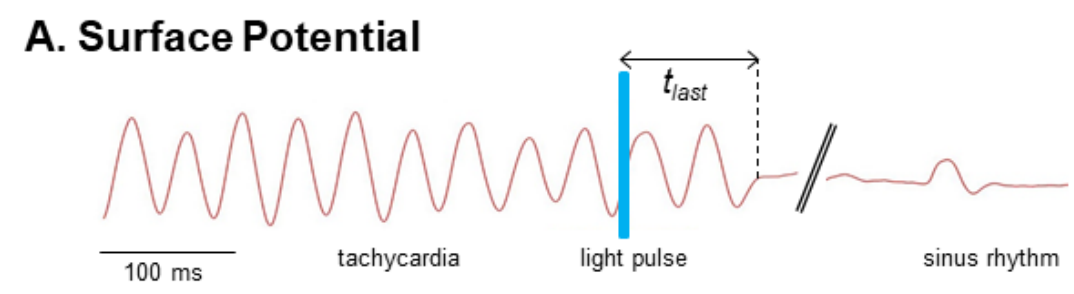

\section{B. Optical Action Potentials}
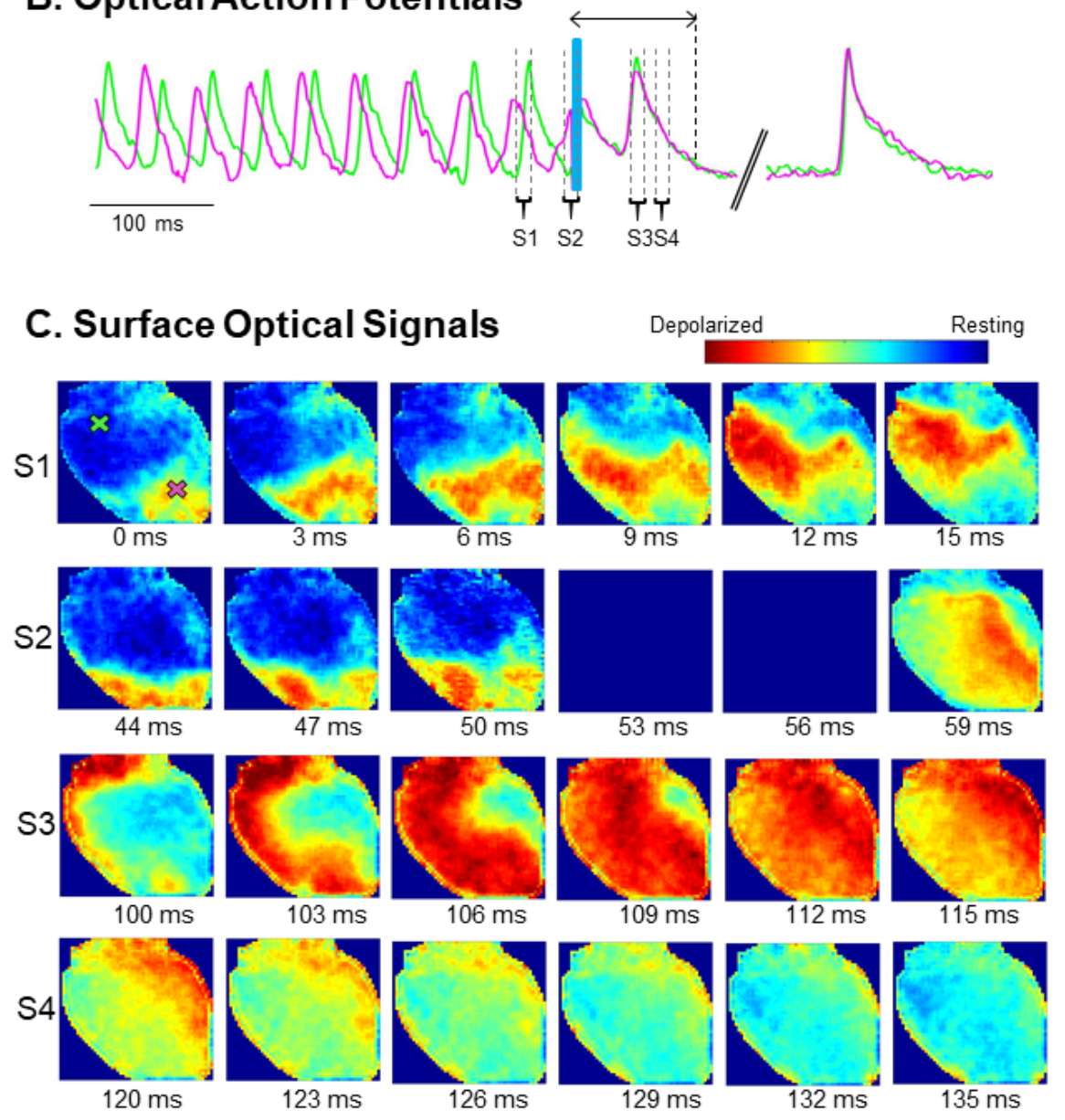

Figure 9.5.: Electrical activity before and during $\boldsymbol{t}_{\text {last }}$. Caption on next page.

In Figure 9.5 A the monomorphic ventricular tachycardia from the pseudo-ECG is observed before being terminated using a single pulse of $2 \mathrm{~ms}$ that leads to the elimination of the arrhythmia after $\sim 1.5$ arrhythmia cycles from the moment of stimulation and the sinus rhythm is restored after a period of $\sim 300 \mathrm{~ms}$ of quiescence. The termination of the arrhythmia as well as $t_{\text {last }}$ are corroborated in Figure 9.5 B where the optical signals of two different pixels are observed. Moreover, these recordings also show that before the stimulation the two sig- 
Figure 9.5.: Electrical activity before and during $\boldsymbol{t}_{\text {last }}$. (A) displays the signal obtained from the surface electrode, representing a pseudo-ECG (red). The ventricular tachycardia disappears after $104 \mathrm{~ms}$ from the beginning of stimulation $\left(t_{\text {last }}\right)$. The blue bar represents the light pulse and the diagonal bars indicate a time lapse of $300 \mathrm{~ms}$. (B) plots the signals of two pixels obtained from the optical mapping recorded, these pixels are marked on the first image of $(\mathbf{C})$, which shows four sequences of images (S1, S2, S3, S4) from before and after the stimulation pulse. The timeline of the sequences is referenced on the plot in (B). S1 and S2 illustrate the arrhythmic wave before stimulation, while S3 the last wave of the arrhythmia before its termination, and S4 the repolarization of the heart after the cease in the arrhythmic activity.

nals displayed different phases but after the pulse of light they are synchronized leading to cardioversion.

Finally, Figure 9.5 $\mathrm{C}$ is composed of different sequences of images, which are the source of the action potentials in Figure 9.5 B. The corresponding pixels of (B) are marked on first image of the top left, and at the same time the sequences S1, S2, S3 and S4 of (C) are marked on the timeplot of (B). In S1 a sequence of activation of the tachycardic can be appreciated, traveling from the apex to the base of the heart. S2 shows part of the next arrhythmia wave before being illuminated. S3 represents the wave traveling on the surface of the heart after stimulation. This wave exhibits a different conformation than the waves prior to stimulation since it is closing in from both the base and the apex of the heart until it covers the whole heart and potentially collides outside of the field of view leading to the cessation of the arrhythmic behavior as observed in S4. The change in the wave's morphology might be caused by the stimulation of the Channelrhodopsin-2 on the excitable gaps leading to their depolarization but also by the opening of channels on the traveling wave that can lead to changes on the membrane potential.

In Figure 9.6 we can see examples from two other hearts. First, in Fig. 9.6 (A \& B) $t_{\text {last }}$ is longer, taking a total of $186 \mathrm{~ms}$. This can be expected as two full arrhythmia cycles continued after illumination, compared to only one full cycle in the previous example. However, what they both have in common is the the change in wave morphology, as the sequence S3 (last depolarization before cardioversion) from the figure displays two wave fronts closing in leading to their collision with each other. This is manifested again as the synchronization in the last action potential of the two pixels analyzed, as also seen in the previous figure.

This same behavior can be appreciated in Fig. 9.6 (C \& D). A change in the traveling pathway of the arrhythmic wave is evident, as it is affected by the light stimulus. Moreover, before illumination the electrical signals coming from the pixels appeared erratic and without a clear period, but they synchronize and exhiit slower dynamics after stimulation. In this case there is only one depolarization wave after the light pulse taking a total $t_{\text {last }}$ of $126 \mathrm{~ms}$. 
A

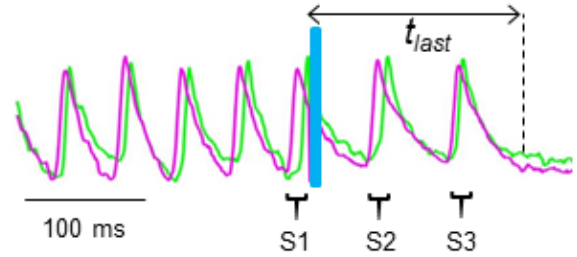

B
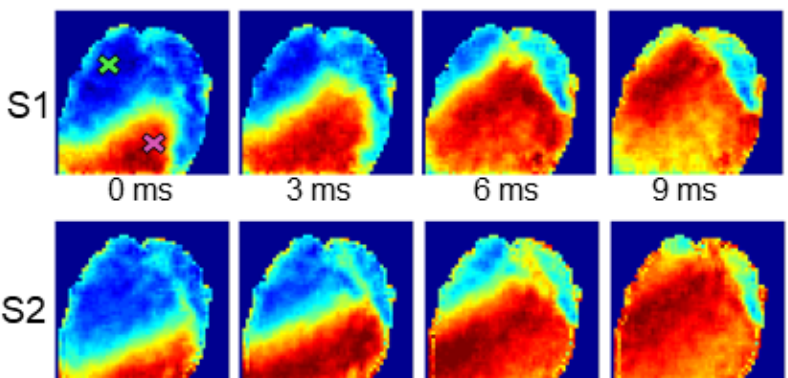

$9 \mathrm{~ms}$

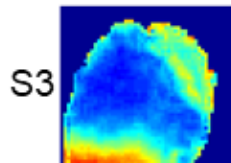

$70 \mathrm{~ms}$

$73 \mathrm{~ms}$

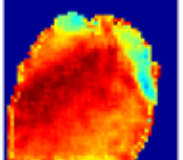

$134 \mathrm{~ms}$
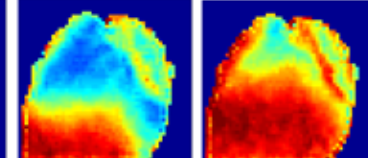

$76 \mathrm{~ms}$

C

$137 \mathrm{~ms}$

$140 \mathrm{~ms}$

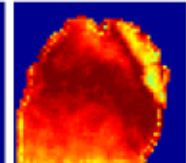

$143 \mathrm{~ms}$
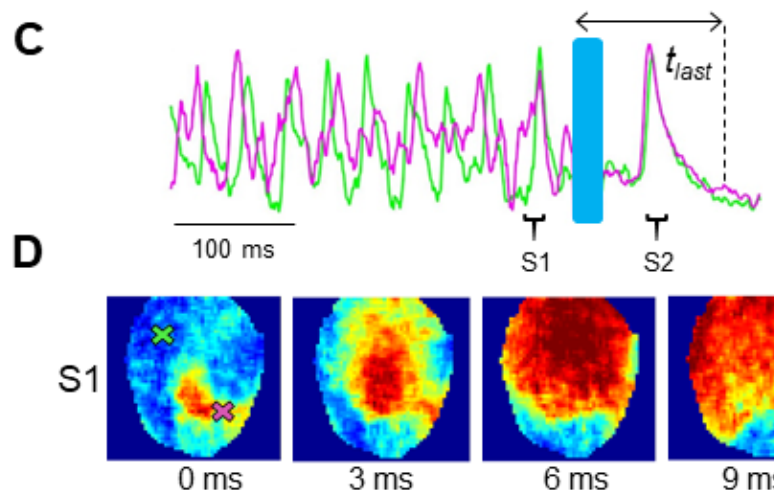

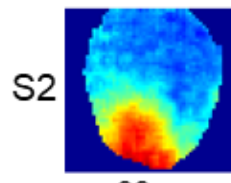

$99 \mathrm{~ms}$

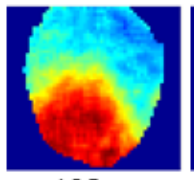

$102 \mathrm{~ms}$
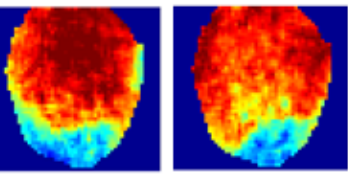

$9 \mathrm{~ms}$

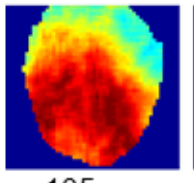

$105 \mathrm{~ms}$

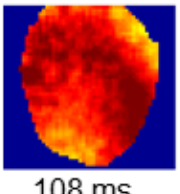

Resting

Figure 9.6.: Arrhythmic wave alteration after illumination. Caption on next page.

\subsection{Discussion \& Conclusions}

Due to the hints of a common mechanism behind global optogenetic cardioversion, I performed a specific analysis of $t_{\text {last }}$ for all the cardioversion cases (Figure 9.2 \& 9.3). Even 
Figure 9.6.: Arrhythmic wave alteration after illumination. Two more examples showing the electrical activity pf the arrhythmic waves before and after optogenetic stimulation. (A) plots the optical action potentials obtained from (B) where three sequences of images are displayed (S1, S2, S3) illustrating the traveling at different time points. In the same manner, $(\mathbf{C})$ plots the optical action potentials obtained from (D).

though pulse length, intensity and arrhythmia morphology will all play a role in determining the success as well as the moment of the cardioversion [12, 19, 67, 61, 88, 60], more than 70\% of the cardioversions materialize in the range between $25 \mathrm{~ms}$ to $100 \mathrm{~ms}$, and approximately $90 \%$ before the $200 \mathrm{~ms}$ mark. This suggests a potential specific process related to this time lapse taking place in order to terminate an arrhythmia. Considering that only 2 out of 417 arrhythmia were terminated at $25 \mathrm{~ms}$ or less, could translate into the process of optogenetic termination needing a minimum amount of time, with these 2 specific cases arising from arrhythmias about to self-terminate.

The minimum necessary time of $t_{\text {last }}$ could originate from the events needed in order to terminate an arrhythmia using optogenetics on global epicardial illumination. Events such as the depolarization of a specific number of cells and the time needed to reach peak ChR-2 currents, both which are intensity dependent, proven to affect $t_{\text {last }}$. At organ scale the mechanisms leading to optogenetic cardioversion are also time-dependent. Creating a conduction block on the path of the arrhythmic wave or generating a new wave to collide with it [76], something that could explain why 1000 ms pulses exhibited shorter and more consistent $t_{\text {last }}$.

As described in all isolated heart ex-vivo optogenetic cardioversion literature [12, 19, 67, 61], increasing the light intensity and pulse width translates into higher termination rates. As seen in our $t_{\text {last }}$ analysis, the same parameters will lead to a higher consistency in the termination times and to a general decrease in this times as seen in the box plots. Therefore, I propose that one of the parameters of reliable (attempts with higher efficiency rates) optogenetic cardioversion techniques should aim to "annihilate" arrhythmias, or in the case of my results, aim at the $25 \mathrm{~ms}$ to $100 \mathrm{~ms}$ range where more than $70 \%$ of the cardioversions took place. Whether high-success-rate attempts lead to faster terminations, or vice-versa is not completely clear but indications show that they are related and probably depend on each other.

The optical mapping analysis of traveling waves demonstrates a modification of the arrhythmic wave as a result of the optogenetic stimulation with a presumable increase in synchronization of the electrical activity in the heart leading to termination. As explained by Sasse and colleagues [76], global stimulation increases the chances of illuminating the excitable gap and creating a new wave that would eventually collide with the arrhythmic wave. Yet another theory that would not exclude a new wave is that stimulation of Channelrhodopsin-2 modifies electrophysiological properties of the cardiac tissue such as the conduction velocity and excitability [15] contributing to the alteration of the wave's morphology and pathwave and aiding 
with its termination. Therefore, taking generally one to two arrhythmia periods after stimulation [67] before the new or modified wave collides, conceivably explaining the typical $t_{\text {last }}$ times manifested. And once again, longer pulses and higher intensities have a greater effect on the cardiac tissue and therefore on the arrhythmic wave leading to faster terminations.

To conclude, the time it takes for an arrhythmia to be terminated using global optogenetic cardioversion has a specific range and it takes place within the first hundreds of milliseconds potentially due to an alteration of the electrophysiological properties of both the excitable and unexcitable tissue. Higher intensities and longer pulses shorten this time, with a higher effect seen by the intensity. Lastly, and as a consequence, the most successful parameters in optogenetic cardioversions displayed shorter termination times.

\subsection{Contributions}

Part of the experiments were performed by the master students Tsima Abou Kors and Vishalini Vinkatesan during their master thesis projects. I completely designed and supervised the project carried on by Tsima, and I partially designed and supervised the project carried on by Vishalini. 


\section{Part III.}

\section{Discussion \& Conclusions}





\section{Discussion}

Cardiac optogenetics is a blooming field, with the first demonstrations of control of cardiac electrophysiology just ten years ago [3, 11] and utility to terminate arrhythmia in isolated heart models showed six years later [12, 61, 19]. However, there is still much to be learned and understood in the cardiac field using photo-sensitive ion channels. This doctoral work focused on the termination of arrhythmia using global illumination on isolated ChR-2 transgenic mice hearts. So far mice hearts have been the most common organ-level model in cardiac optogenetics and channelrhodopsin-2 the main optogenetic tool implemented. Previous work done from Bruegmann and Crocini [12, 19] set the foundations of this research project. Almost simultaneously Bruegmann tested different stimulation areas, intensities and pulse lengths showing the feasibility of optogenetic cardioversion, while Crocini implemented a mechanisitic design of illumination patterns to stop arrhymthic activity making use of the spatiotemporal advantages of optogenetics. Therefore, testing a different method to stimulate the heart in an attempt to terminate arrhythmias would offer new insights and allow comparisons with the already obtained knowledge and provide perspectives that can help improve the general understanding of optogenetic arrhythmia termination. This discussion section gives a general perspective and potential future experiments, since discussions specific to the results obtained have been included in each section.

There are some clear advantages and disadvantages of applying global epicardial illumination to terminate arrhythmias. As demonstrated by various groups, irradiation of a larger area will require lower intensities and shorter pulses to produce an optogenetic response [96, 19, 67, 11]. The closer optogenetic cardioversion gets to the clinics, the greater the importance of terminating arrhythmias with lower intensity. As seen in our light attenuation results, the blood and other heart structures greatly decrease the amount of light penetrating the deepest layers of the cardiac walls. Therefore, achieving optogenetic cardioversion in larger animal models and in humans will be a matter of light delivery as already shown in different in-silico models [41, 12, 9]. On the other hand, global illumination comes with the technical challenge of designing a light delivery method in-vivo.

Up to date, optogenetic control has been shown in zebrafish [3], mouse [11, 12, 19, 96 , 67, 17, 29, 98, 13, 85] and rat hearts [61, 60, 62], all of them representing small animal models. Therefore it is safe to assume that optogenetics in larger hearts will come with new physical and physiological challenges. However, studies like the one made during this doctoral project give a head start to future investigations. As shown here and in other results, pacing and cardioversion depend on the area illuminated, the intensity applied and the length of the pulse. 
This can be translated as the volume of cardiac tissue/cardiomyocytes excited over a period of time. The volume excited will also depend on the sensitivity of the opsin used [55], the excitation wavelength and on the attenuation properties of the areas of the heart excited.

The analysis of $t_{\text {last }}$ shows a potential common mechanism behind optogenetic cardioversion using global illumination. This mechanism might be specific to the combination of ChR-2 in a mouse heart stimulated with global illumination but at the same time a similar behavior can be expected from other species. A retrospective analysis of $t_{\text {last }}$ from different illumination methods tested by other groups would not require extensive work nor further experiments and might complement this study and provide data that could strengthen this theory. Since $t_{\text {last }}$ is affected by the pulse width and intensity, we can assume that the area illuminated will also play a role and that smaller areas will take longer to terminate an arrhythmia but we will still find a constant or common termination time like the one identified in this project.

While no clear effect of the morphology on the efficiency of optogenetic arrhythmia termination was identified, further experiments could bring deeper understanding to the differences. Additionally, mice hearts are less suitable to host arrhythmia [25, 38] and larger animal studies might be better in clarifying this theory. In a translation to clinics scenario, patients with identified arrhythmia would benefit from the characterization of optogenetic energy requirements of different kinds of arrhythmia since patient specific treatments could be designed.

Last but not least, while other groups have already explored patterned [77, 19] and intramural [98] illumination on isolated hearts, this project aimed to investigate the general and so far unexplored scenario of global epicardial illumination. Answers obtained from this method aid prospective research take full advantage of the high spatial resolution offered by optogenetics.

The results presented in this doctoral research project have firstly provided new insights into cardiac optogenetic stimulation, and secondly brought new ideas to explore in the application of optogenetics to investigate, control and terminate cardiac arrhythmia. 


\section{Conclusions}

In this thesis work I have focused my research on understanding global optogenetic stimulation as a means to terminate arrhythmia on isolated mice hearts expressing ChR-2. I hypothesized that compared to local illumination, global illumination would reduce the intensity and the pulse widths necessary to terminate an arrhythmia and would also improve our knowledge of the mechanisms involved. This research project has covered four different aspects: first understanding optogenetic stimulation in this mouse model under various conditions, followed by optogenetic arrhythmia termination using global stimulation, to move into specifics of global optogenetic cardioversion such as the efficiency of monomorphic and polymorphic termination attempts and lastly analyzing the time it takes to terminate an arrhythmia and the factors behind it.

From the first task we can conclude that laboratory chemicals such as dyes and electromechanical uncouplers increase the attenuation of light and will have an effect on optogenetic stimulation thresholds. Similarly, a heart filled with blood will present higher attenuation compared to a heart perfused with tyrode, the perfusion solution used in most ex-vivo experiments. Moreover, stimulating the heart requires a minimum energy that will depend on the pulse length, the intensity of the delivered light and the surface area illuminated. Therefore, by increasing one of these parameters the others can be decreased and the other way around. The pacing threshold will also depend on the specific area of the heart stimulated, with the left ventricle showing a higher threshold than the right ventricle.

Similar to pacing, the success of optogenetic termination using global stimulation will depend on intensity, area and pulse width. However, the energy required to terminate an arrhythmia will be larger by at least one order of magnitude. Illuminating the whole epicardial surface provided us the advantage of decreasing both intensity and total stimulation time, reaching high success rates with single pulses of $10 \mathrm{~ms}$ to $1000 \mathrm{~ms}$ using intensities lower than the intensities needed to pace using optical fibers. Combined with these experiments we also analyzed for the first occasion the time needed to terminate an arrhythmia $\left(t_{\text {last }}\right)$. Our observations showed that optogenetic cardioversions performed with higher intensities eliminated arrhythmias sooner, and that the majority of cardioversions took place on the onset of stimulation with a small percentage happening later. Both events were visible on the surface of the heart using the optical mapping technique, confirming that stimulation of the excitable gaps leads to faster cardioversions while slower ones could be a result of a perturbation of the arrhythmia. 
The ratio of arrhythmia termination based on morphology did not give a clear answer on the differences in termination success between monomorphic and polymorphic arrhythmia. While long pulses of $1000 \mathrm{~ms}$ were able to terminate monomorphic ventricular tachycardia even with low intensities, for $100 \mathrm{~ms}$ and $10 \mathrm{~ms}$ no significant differences were found. Since the number of polymorphic VT measured was lower, a better comparison can be made by increasing the number of samples. Both morphology analysis and arrhythmia cycle length analysis offer interesting points for deeper future investigations.

I introduced the term cardioversion time $\left(t_{\text {last }}\right)$, and its analysis led to a clear time lapse for optogenetic arrhythmia termination using global stimulation. $87 \%$ materialize $\leq 200 \mathrm{~ms}$ revealing the possibility of a common mechanism behind optogenetic cardioversion. Optical recordings of the traveling waves on the surface of the heart suggest an alteration of the arrhythmic wave leading to its collision. Moreover, $t_{\text {last }}$ decreased and became more consistent for higher stimulation intensities and to a lower degree for longer pulse widths.

The research done on different aspects of global illumination as method of optogenetic cardioversion broadens the understanding, advantages and limitations of optogenetics as a tool to control and investigate arrhythmias. It answers physical and physiological questions and opens new research questions, both of clinical relevance setting the ground for investigation in large animal models. 


\section{Bibliography}

[1] Oscar J. Abilez, Jonathan Wong, Rohit Prakash, Karl Deisseroth, Christopher K. Zarins, and Ellen Kuhl. Multiscale computational models for optogenetic control of cardiac function. Biophysical Journal, 101(6):1326-1334, 2011. ISSN 00063495. doi: 10.1016/ j.bpj.2011.08.004. URL http://dx.doi.org/10.1016/j.bpj.2011.08.004.24,25, 27

[2] Tsima Abou Kors. Ventricle Specific Cardioversion upon Optogenetic Photostimulation, 2019. 48

[3] Stainier D. Arrenberg A. Optogenetic Control of Cardiac Function. Science, 330 (November):971-974, 2010. 95

[4] Franz Baudenbacher, James D Potter, C Björn, Franz Baudenbacher, Tilmann Schober, Jose Renato Pinto, Veniamin Y Sidorov, Fredrick Hilliard, R John Solaro, James D Potter, and Björn C Knollmann. Myofilament Ca $2+$ sensitization causes susceptibility to cardiac arrhythmia in mice Find the latest version : Myofilament $\mathrm{Ca} 2+$ sensitization causes susceptibility to cardiac arrhythmia in mice. The Journal of Clinical Investigation, 118(12):3893-3903, 2008. doi: 10.1172/JCI36642.been. 73

[5] Robert M. Bell, Mihaela M. Mocanu, and Derek M. Yellon. Retrograde heart perfusion: The Langendorff technique of isolated heart perfusion. Journal of Molecular and Cellular Cardiology, 50(6):940-950, 2011. ISSN 00222828. doi: 10.1016/j.yjmcc.2011.02. 018. URL http://dx.doi.org/10.1016/j.yjmcc.2011.02.018, 17, 18, 47

[6] Brian O. Bingen, Marc C. Engels, Martin J. Schalij, Wanchana Jangsangthong, Zeinab Neshati, Iolanda Feola, Dirk L. Ypey, Said F A Askar, Alexander V. Panfilov, Daniel A. Pijnappels, and Antoine A F De Vries. Light-induced termination of spiral wave arrhythmias by optogenetic engineering of atrial cardiomyocytes. Cardiovascular Research, 104 (1):194-205, 2014. ISSN 17553245. doi: 10.1093/cvr/cvu179. 27, 28

[7] Ryan T. Borne, Paul D. Varosy, and Frederick A. Masoudi. Implantable CardioverterDefibrillator Shocks: Epidemiology, Outcomes, and Therapeutic Approaches. JAMA Internal Medicine, 173(10):859-865, 05 2013. ISSN 2168-6106. doi: 10.1001/ jamainternmed.2013.428. URL https://doi.org/10.1001/jamainternmed.2013. 428. 19 
[8] Ryan T. Borne, Paul D. Varosy, and Frederick A. Masoudi. Implantable CardioverterDefibrillator Shocks: Epidemiology, Outcomes, and Therapeutic Approaches. JAMA Internal Medicine, 173(10):859-865, 05 2013. ISSN 2168-6106. doi: 10.1001/ jamainternmed.2013.428. URL https://doi.org/10.1001/jamainternmed.2013. 428. 19

[9] Patrick M Boyle, John C Williams, Christina M Ambrosi, Emilia Entcheva, and Natalia a Trayanova. A comprehensive multiscale framework for simulating optogenetics in the heart. Nature communications, 4:2370, 2013. ISSN 2041-1723. doi: 10.1038/ ncomms3370. URL http://www.nature.com/ncomms/2013/130828/ncomms3370/ full/ncomms3370.html. 25, 46, 47, 95

[10] Kieran E. Brack, Ravi Narang, James Winter, and G. André Ng. The mechanical uncoupler blebbistatin is associated with significant electrophysiological effects in the isolated rabbit heart. Experimental Physiology, 98(5):1009-1027, 2013. ISSN 09580670. doi: 10.1113/expphysiol.2012.069369. 54,73

[11] Tobias Bruegmann, D Malan, M Hesse, T Beiert, C J Fuegemann, B K Fleischmann, and P Sasse. Optogenetic control of heart muscle in vitro and in vivo. Nature methods, 7 (11):897-900, 2010. ISSN 1548-7105. doi: 10.1038/nmeth.1512. URL http://www . ncbi.nlm.nih.gov/pubmed/20881965, 23, 25, 27, 46, 95

[12] Tobias Bruegmann, Patrick M. Boyle, Christoph C. Vogt, Thomas V. Karathanos, Hermenegild J. Arevalo, Bernd K. Fleischmann, Natalia A. Trayanova, and Philipp Sasse. Optogenetic defibrillation terminates ventricular arrhythmia in mouse hearts and human simulations. Journal of Clinical Investigation, 126(10):3894-3904, 2016. ISSN 15588238. doi: 10.1172/JCI88950. 1, 22, 28, 29, 39, 47, 57, 73, 91, 95

[13] Tobias Bruegmann, Thomas Beiert, Christoph C. Vogt, Jan W. Schrickel, and Philipp Sasse. Optogenetic termination of atrial fibrillation in mice. Cardiovascular Research, 114(5):713-723, 2018. ISSN 17553245. doi: 10.1093/cvr/cvx250. 95

[14] Francis L Burton and Stuart M Cobbe. Dispersion of ventricular repolarization and refractory period. Cardiovascular Research, 50:10-23, 2001. 11, 15

[15] Rebecca A. B. Burton, Aleksandra Klimas, Christina M. Ambrosi, Jakub Tomek, Alex Corbett, Emilia Entcheva, and Gil Bub. Optical control of excitation waves in cardiac tissue. Nature Photonics, 9(12):813-816, 2015. ISSN 1749-4885. doi: 10.1038/ nphoton.2015.196. URL http://www.pubmedcentral.nih.gov/articlerender. fcgi?artid=4821438\{\&\}tool=pmcentrez $\{\&\}$ rendertype=abstract, 25, 27, 73, 91

[16] G. Chaudrhy, MD Muqtada, and Charles Haffajee. Antiarrhythmic agents and proarrhythmia. Critical Care Medicine, 28(10):158-164, 2000. 19 
[17] Yue Cheng, Haitao Li, Hong Lei, Chan Jiang, Panpan Rao, Long Wang, Fang Zhou, and Xi Wang. Flexible and precise control of cardiac rhythm with blue light. Biochemical and Biophysical Research Communications, 514(3):759-764, 2019. ISSN 10902104. doi: 10.1016/j.bbrc.2019.05.035. URL https://doi.org/10.1016/j.bbrc.2019. 05.035. 25, 95

[18] J. Christoph, J. Schröder-Schetelig, and S. Luther. Electromechanical optical mapping. Progress in Biophysics and Molecular Biology, 130:150-169, 2017. ISSN 00796107. doi: 10.1016/j.pbiomolbio.2017.09.015. 40

[19] Claudia Crocini, Cecilia Ferrantini, Raffaele Coppini, Marina Scardigli, Ping Yan, Leslie M Loew, Godfrey Smith, Elisabetta Cerbai, Corrado Poggesi, Francesco S Pavone, and Leonardo Sacconi. Optogenetics design of mechanistically-based stimulation patterns for cardiac defibrillation. Nature Publishing Group, pages 1-7, 2016 . doi: 10.1038/srep35628. URL http://dx.doi.org/10.1038/srep35628, 1, 22, 28, 30, 46, 55, 57, 73, 91, 95, 96

[20] Claudia Crocini, Cecilia Ferrantini, Francesco S Pavone, and Leonardo Sacconi. Optogenetics gets to the heart: A guiding light beyond defibrillation. Progress in Biophysics and Molecular Biology, 1:1-8, 2017. ISSN 0079-6107. doi: 10.1016/j.pbiomolbio.2017. 05.002. URL http://dx.doi.org/10.1016/j.pbiomolbio.2017.05.002, 30

[21] Jorge M. Davtdenko. Spiral wave activity:. Journal of Cardiovascular Electrophysiology, 4(6):730-746, 1993. doi: 10.1111/j.1540-8167.1993.tb01258. x. URL https://onlinelibrary.wiley.com/doi/abs/10.1111/j.1540-8167. 1993.tb01258.x. 75

[22] Karl Deisseroth, Guoping Feng, Ania K. Majewska, Gero Miesenböck, Alice Ting, and Mark J. Schnitzer. Next-generation optical technologies for illuminating genetically targeted brain circuits. Journal of Neuroscience, 26(41):10380-10386, 2006. ISSN 02706474. doi: 10.1523/JNEUROSCI.3863-06.2006. 21

[23] Berengere M. Dumotier. A straightforward guide to the basic science behind arrhythmogenesis. Postgraduate Medical Journal, 91(1074):221-229, 2015. ISSN 14690756. doi: 10.1136/postgradmedj-2014-305647rep. 14,15

[24] Mario Dvorkin and Daniel Cardinali. Best \& Taylor : Bases Fisiológicas de la práctica Médica. Editorial Médica Panamericana, 13 edition, 2005. ISBN 9500602431. 5, 6

[25] Andrew G Edwards and William E Louch. Species-Dependent Mechanisms of Cardiac Arrhythmia : A Cellular Focus. Clinical Medicine Insights: Cardiology, 2017. doi: 10.1177/1179546816686061. 96

[26] Emilia Entcheva. Cardiac optogenetics. American journal of physiology. Heart and cir- 
culatory physiology, 304(9):H1179-91, 2013. ISSN 1522-1539. doi: 10.1152/ajpheart. 00432.2012. URL http://www.pubmedcentral.nih.gov/articlerender.fcgi? artid=3652095 $\{\&\}$ tool=pmcentrez $\{\&\}$ rendertype=abstract, 23,24

[27] Christopher L.-H. Ferenczi, Emily A; Tan, Xiaoqiu; Huang. Principles of Optogenetic Methods and Their Application to Cardiac Experimental Systems. Frontiers in Physiology, 10(September), 2019. doi: 10.3389/fphys.2019.01096. 73

[28] Thomas P. Flagg and Colin G. Nichols. "Cardiac $K_{A T P}$ ": A Family of Ion Channels. Circulation: Arrhythmia and Electrophysiology, 4(6):796-798, 2011. doi: 10.1161/ CIRCEP.111.968081. 28

[29] Maximilian Funken, Daniela Malan, Philipp Sasse, and Tobias Bruegmann. Optogenetic Hyperpolarization of Cardiomyocytes Terminates Ventricular Arrhythmia. Frontiers in Physiology, 10(April):1-7, 2019. doi: 10.3389/fphys.2019.00498. URL https://www . frontiersin.org/article/10.3389/fphys.2019.00498/full. 95

[30] Alexey V Glukhov, Thomas P Flagg, Vadim V Fedorov, R E Igor, and Colin G Nichols. Differential KATP channel pharmacology in intact mouse heart. Journal of Molecular and Cellular Cardiology, 48:152-160, 2010. doi: https://doi.org/10.1016/j.yjmcc.2009. 08.026. 39

[31] Du guan Fu. Cardiac Arrhythmias: Diagnosis, Symptoms, and Treatments. Cell Biochemistry and Biophysics, 73(2):291-296, 2015. ISSN 10859195. doi: 10.1007/ s12013-015-0626-4. URL http://dx.doi.org/10.1007/s12013-015-0626-4. 19

[32] Arthur Guyton and J.E. Hall. Tratado de Fisiología Médica. Elsevier Espana, 11 edition, 2006. ISBN 9788481749267. 5, 6, 8, 9

[33] Christopher L.-H. Huang. Murine Electrophysiological Models of Cardiac Arrhythmogenesis. Physiological Reviews, 97(1):283-409, jan 2017. ISSN 0031-9333. doi: 10.1152/physrev.00007.2016. URL http://www.physiology.org/doi/10.1152/ physrev.00007.2016, 9, 12, 14, 15

[34] Paul A. Iaizzo. Handbook of cardiac anatomy, physiology, and devices. SPRINGER, 2016. doi: 10.1007/978-3-319-19464-6. 7, 8, 9

[35] Grigory Ikonnikov and Eric Wong. Action potential of cardiac muscles, 2013. URL http://www.pathophys.org/ physiology-of-cardiac-conduction-and-contractility/actionpotential/. Accessed: 2020-Feb-18. 10

[36] Steven L. Jacques. Optical properties of biological tissues: A review. Physics in Medicine and Biology, 58(11), 2013. ISSN 00319155. doi: 10.1088/0031-9155/58/ 
11/R37. 46

[37] Roy M. John, Usha B. Tedrow, Bruce A. Koplan, Christine M. Albert, Laurence M. Epstein, Michael O. Sweeney, Amy Leigh Miller, Gregory F. Michaud, and William G. Stevenson. Ventricular arrhythmias and sudden cardiac death. The Lancet, 380(9852): 1520-1529, 2012. ISSN 1474547X. doi: 10.1016/S0140-6736(12)61413-5. URL http: //dx.doi.org/10.1016/S0140-6736(12)61413-5, 13, 19, 75

[38] Sven Kaese, Sander Verheule, and Yoram Etzion. Cardiac electrophysiology in mice : a matter of size. Frontiers in Physiology, 3(September):1-19, 2012. doi: 10.3389/fphys. 2012.00345. 96

[39] Asli Kalin, Juliet Usher-Smith, Victoria J. Jones, Christopher L.H. Huang, and Ian N. Sabir. Cardiac Arrhythmia: A Simple Conceptual Framework. Trends in Cardiovascular Medicine, 20(3):103-107, 2010. ISSN 10501738. doi: 10.1016/j.tcm.2010.09.003. URL http://dx.doi.org/10.1016/j.tcm.2010.09.003. 14, 15

[40] Vineesh Kappadan, Saba Telele, Ilija Uzelac, Flavio Fenton, Ulrich Parlitz, Stefan Luther, and Jan Christoph. High-resolution optical measurement of cardiac restitution, contraction, and fibrillation dynamics in beating vs. blebbistatin-uncoupled isolated rabbit hearts. Frontiers in Physiology, 11:464, 2020. ISSN 1664-042X. doi: 10. 3389/fphys.2020.00464. URL https://www.frontiersin.org/article/10.3389/ fphys. 2020.00464. 54

[41] Thomas V Karathanos, Jason D Bayer, Dafang Wang, Patrick M Boyle, and Natalia A Trayanova. Opsin spectral sensitivity determines the effectiveness of optogenetic termination of ventricular fibrillation in the human heart: a simulation study. The Journal of physiology, 594(23):6879-6891, 2016. ISSN 1469-7793. doi: 10.1113/ JP271739. URL http://www.ncbi.nlm.nih.gov/pubmed/26941055http://www. pubmedcentral.nih.gov/articlerender.fcgi?artid=PMC5134403, 32, 33, 35, 47, 95

[42] R E Kerber, M G Kienzle, B Olshansky, A L Waldo, D Wilber, M D Carlson, A M Aschoff, S Birger, L Fugatt, and S Walsh. Ventricular tachycardia rate and morphology determine energy and current requirements for transthoracic cardioversion. Circulation, 85(1):158-163, 1992. doi: 10.1161/01.CIR.85.1.158. URL https://www. ahajournals.org/doi/abs/10.1161/01.CIR.85.1.158, 76

[43] M. J. Killeen, G. Thomas, I. S. Gurung, C. A. Goddard, J. A. Fraser, M. P. Mahaut-Smith, W. H. Colledge, A. A. Grace, and C. L.H. Huang. Arrhythmogenic mechanisms in the isolated perfused hypokalaemic murine heart. Acta Physiologica, 189(1):33-46, 2007. ISSN 17481708. doi: 10.1111/j.1748-1716.2006.01643.x. 39

[44] James H. King, Christopher L.H. Huang, and James A. Fraser. Determinants of myocar- 
dial conduction velocity: Implications for arrhythmogenesis. Frontiers in Physiology, 4 JUN(June):1-14, 2013. ISSN 1664042X. doi: 10.3389/fphys.2013.00154. 17

[45] Sonja Kleinlogel, Katrin Feldbauer, Robert E. Dempski, Heike Fotis, Phillip G. Wood, Christian Bamann, and Ernst Bamberg. Ultra light-sensitive and fast neuronal activation with the Ca 2+-permeable channelrhodopsin CatCh. Nature Neuroscience, 14(4):513518, 2011. ISSN 10976256. doi: 10.1038/nn.2776. 27

[46] J. Kolega. Phototoxicity and photoinactivation of blebbistatin in UV and visible light. Biochemical and Biophysical Research Communications, 320(3):1020-1025, jul 2004. ISSN 0006291X. doi: 10.1016/j.bbrc.2004.06.045. URL https://linkinghub. elsevier.com/retrieve/pii/S0006291X04013233. 54

[47] Gail K Larsen, John Evans, William E Lambert, Yiyi Chen, and Merritt H Raitt. Shocks burden and increased mortality in implantable cardioverter-defibrillator patients. HRTHM, 8(12):1881-1886, 2011. ISSN 1547-5271. doi: 10.1016/j.hrthm.2011.07.036. URL http://dx.doi.org/10.1016/j.hrthm.2011.07.036. 19

[48] Eniko Lazar, Hesham A Sadek, and Olaf Bergmann. Cardiomyocyte renewal in the human heart : insights from the fall-out $\mathrm{z}$ a Cardiomyocyte turnover. European Heart Journal, pages 2333-2339, 2017. doi: 10.1093/eurheartj/ehx343. 6

[49] George Guan-hua Lin and Jeffrey G Scott. Illunimating cell signaling with optogenetic tools. Nat Rev Mol Cell Biol, 100(2):130-134, 2012. ISSN 15378276. doi: 10.1016/j. pestbp.2011.02.012.Investigations. 21

[50] Rupamanjari Majumder, Iolanda Feola, Alexander S. Teplenin, Antoine Af de Vries, Alexander V. Panfilov, and Daniel A. Pijnappels. Optogenetics enables real-time spatiotemporal control over spiral wave dynamics in an excitable cardiac system. eLife, 7: 1-17, 2018. ISSN 2050084X. doi: 10.7554/eLife.41076. 22,73

[51] Matteo E Mangoni. Genesis and Regulation of the Heart Automaticity. Physiological Reviews, pages 919-982, 2008. doi: 10.1152/physrev.00018.2007. 7

[52] Gregory M. Marcus, Derrick W. Chan, and Rita F. Redberg. Recollection of pain due to inappropriate versus appropriate implantable cardioverter-defibrillator shocks. PACE - Pacing and Clinical Electrophysiology, 34(3):348-353, 2011. ISSN 01478389. doi: 10.1111/j.1540-8159.2010.02971.x. 19

[53] Claire A. Martin, Laila Guzadhur, Andrew A. Grace, Ming Lei, and Christopher L.H. Huang. Mapping of reentrant spontaneous polymorphic ventricular tachycardia in a scn5a $+/$ - mouse model. American Journal of Physiology-Heart and Circulatory Physiology, 300(5):H1853-H1862, 2011. doi: 10.1152/ajpheart.00034.2011. URL https://doi.org/10.1152/ajpheart.00034.2011, PMID: 21378142. 76 
[54] Arvydas Matiukas, Bogdan G. Mitrea, Maochun Qin, Arkady M. Pertsov, Alexander G. Shvedko, Mark D. Warren, Alexey V. Zaitsev, Joseph P. Wuskell, Mei-de Wei, James Watras, and Leslie M. Loew. Near-infrared voltage-sensitive fluorescent dyes optimized for optical mapping in blood-perfused myocardium. Heart Rhythm, 4(11): 1441-1451, nov 2007. ISSN 15475271. doi: 10.1016/j.hrthm.2007.07.012. URL https://linkinghub.elsevier.com/retrieve/pii/S1547527107007163, 47, 48

[55] Joanna Mattis, Kay M Tye, Emily A Ferenczi, Charu Ramakrishnan, Daniel J O'Shea, Rohit Prakash, Lisa A Gunaydin, Minsuk Hyun, Lief E Fenno, Viviana Gradinaru, Ofer Yizhar, and Karl Deisseroth. Principles for applying optogenetic tools derived from direct comparative analysis of microbial opsins. Nature Methods, 9(2):159-172, feb 2012. ISSN 1548-7091. doi: 10.1038/nmeth.1808. URL http://www . nature.com/ articles/nmeth.1808, 21,96

[56] Harvey J Motulsky and Ronald E Brown. Detecting outliers when fitting data with nonlinear regression - a new method based on robust nonlinear regression and the false discovery rate. BMC Bioinformatics, 20:1-20, 2006. doi: 10.1186/1471-2105-7-123. URL https://doi .org/10.1186/1471-2105-7-123, 42

[57] Georg Nagel, Tanjef Szellas, Wolfram Huhn, Suneel Kateriya, Nona Adeishvili, Peter Berthold, Doris Ollig, Peter Hegemann, and Ernst Bamberg. Channelrhodopsin-2, a directly light-gated cation-selective membrane channel. Proceedings of the National Academy of Sciences, 100(24):13940-13945, 2003. ISSN 0027-8424. doi: 10.1073/ pnas.1936192100. URL https://www.pnas.org/content/100/24/13940. 23

[58] Georg Nagel, Martin Brauner, Jana F. Liewald, Nona Adeishvili, Ernst Bamberg, and Alexander Gottschalk. Light activation of Channelrhodopsin-2 in excitable cells of caenorhabditis elegans triggers rapid behavioral responses. Current Biology, 15(24): 2279-2284, 2005. ISSN 09609822. doi: 10.1016/j.cub.2005.11.032. 21

[59] Jeanne M Nerbonne and Robert S Kass. Molecular physiology of cardiac repolarization. Science's STKE, 85(4):1205, 2005. doi: 10.1152/physrev.00002.2005. 9, 12

[60] Udi Nussinovitch and Lior Gepstein. Optogenetics for in vivo cardiac pacing and resynchronization therapies. Nature Biotechnology, 33(7):750-754, 2015. ISSN 1546-1696. doi: 10.1038/nbt.3268. URL http://www . nature.com/doifinder/10.1038/nbt. 3268. 46, 55, 91, 95

[61] Emile C.A. Nyns, Annemarie Kip, Cindy I. Bart, Jaap J. Plomp, Katja Zeppenfeld, Martin J. Schalij, Antoine A.F. de Vries, and Daniël A. Pijnappels. Optogenetic termination of ventricular arrhythmias in the whole heart: towards biological cardiac rhythm management. European Heart Journal, page ehw574, 2016. ISSN 0195668X. doi: 10.1093/eurheartj/ehw574. URL http://eurheartj . oxfordjournals. org/lookup/doi/10.1093/eurheartj/ehw574, 1, 28, 33, 57, 76, 91, 95. 
[62] Emile C.A. Nyns, René H Poelma, Linda Volkers, Jaap J Plomp, Cindy I Bart, Annemarie M Kip, Thomas J Van Brakel, Katja Zeppenfeld, Martin J Schalij, Guo Qi Zhang, Antoine A F De Vries, and Daniël A Pijnappels. An automated hybrid bioelectronic system for autogenous restoration of sinus rhythm in atrial fibrillation. Science Translational Medicine, pages 1-12, 2019. ISSN 1946-6234. doi: 10.1126/scitranslmed. aau6447. 95

[63] Christopher O’Shea, Andrew Phillip Holmes, James Winter, Joao Correia, Xianhong $\mathrm{Ou}$, Ruirui Dong, Shicheng He, Paulus Kirchhof, Larissa Fabritz, Kashif Rajpoot, and Davor Pavlovic. Cardiac optogenetics and optical mapping - Overcoming spectral congestion in all-optical cardiac electrophysiology. Frontiers in Physiology, 10 (March):182, 2019. ISSN 1664-042X. doi: 10.3389/FPHYS.2019.00182. URL https: //www.frontiersin.org/articles/10.3389/fphys.2019.00182/abstract. 18 , 54,73

[64] Erika Pastrana. Optogenetics: controlling cell function with light. Nature Methods, 8 (1):24-25, 2011. ISSN 1548-7091. doi: 10.1038/nmeth.f.323. 21

[65] Robert Plonsey and Robert C. Barr. Bioelectricity. A quantitative approach. SPRINGER, 2007. doi: 10.1007/978-1-4419-6658-2. 7, 8

[66] Zhilin Qu and James N. Weiss. Mechanisms of Ventricular Arrhythmias: From Molecular Fluctuations to Electrical Turbulence. Annual Review of Physiology, 77(1):29-55, 2015. ISSN 0066-4278. doi: 10.1146/annurev-physiol-021014-071622. 14, 15, 17

[67] Raúl A. Quiñonez Uribe, Stefan Luther, Laura Diaz-Maue, and Claudia Richter. EnergyReduced Arrhythmia Termination Using Global Photostimulation in Optogenetic Murine Hearts. Frontiers in Physiology, 9(November):1-12, 2018. ISSN 1664-042X. doi: 10. 3389/fphys.2018.01651. URL https://www.frontiersin.org/article/10.3389/ fphys.2018.01651/full, 22, 31, 39, 76, 80, 83, 87, 91, 92, 95

[68] Martin L. Rein and Jan M. Deussing. The optogenetic (r)evolution. Molecular Genetics and Genomics, 287(2):95-109, 2012. ISSN 16174615. doi: 10.1007/ s00438-011-0663-7. 22

[69] Claudia Richter and Tobias Bruegmann. No light without the dark: Perspectives and hindrances for translation of cardiac optogenetics. Progress in Biophysics and Molecular Biology, 2019. 19, 31, 73

[70] Claudia Richter, Jan Christoph, Stephan E Lehnart, and Stefan Luther. Optogenetic Light Crafting Tools for the Control of Cardiac Arrhythmias. In Optogenetics. Methods and Protocols., volume 1408, pages 293-302. Humana Press, New York, NY, 2016. ISBN 978-1-4939-3510-9. doi: 10.1007/978-1-4939-3512-3. URL http://link . springer. com/10.1007/978-1-4939-3512-3. 37 
[71] Andre Roggan, Moritz Friebel, Klaus Doerschel, Andreas Hahn, and Gerhard J. Mueller. Optical properties of circulating human blood in the wavelength range 400-2500 nm. Journal of Biomedical Optics, 4(1):36 - 46, 1999. doi: 10.1117/1.429919. URL https: //doi.org/10.1117/1.429919. 47,54

[72] Kristina Ropella. Introduction to Statistics for Biomedical Engineers, volume 2. Morgan \& Claypool, 01 2007. doi: 10.2200/S00095ED1V01Y200708BME014. 13, 41

[73] Shigeyoshi Saito, Kasumi Masuda, Yuki Mori, Satoshi Nakatani, Yoshichika Yoshioka, and Kenya Murase. Mapping of left ventricle wall thickness in mice using 11.7-T magnetic resonance imaging. Magnetic Resonance Imaging, 36:128-134, 2017. ISSN 18735894. doi: 10.1016/j.mri.2016.10.030. URL http://dx.doi.org/10.1016/j. mri.2016.10.030. 50

[74] Takeshi Sakamoto, John Limouze, Christian A. Combs, Aaron F. Straight, and James R. Sellers. Blebbistatin, a myosin ii inhibitor, is photoinactivated by blue light. Biochemistry, 44(2):584-588, 2005. doi: 10.1021/bi0483357. URL https://doi.org/10. 1021/bi0483357. PMID: 15641783. 47, 54

[75] Faramarz H Samie and José Jalife. Mechanisms underlying ventricular tachycardia and its transition to ventricular fibrillation in the structurally normal heart. Cardiovascular Research, 50(2):242-250, 05 2001. ISSN 0008-6363. doi: 10.1016/S0008-6363(00) 00289-3. URL https://doi.org/10.1016/S0008-6363(00)00289-3. 75

[76] Philipp Sasse, Maximilian Funken, Thomas Beiert, and Tobias Bruegmann. Optogenetic Termination of Cardiac Arrhythmia: Mechanistic Enlightenment and Therapeutic Application? Frontiers in Physiology, 10(June), 2019. ISSN 1664-042X. doi: 10.3389/fphys.2019.00675. URL https://www.frontiersin.org/article/ 10.3389/fphys.2019.00675/full, 21, 31, 32, 91

[77] M. Scardigli, C. Müllenbroich, E. Margoni, S. Cannazzaro, C. Crocini, C. Ferrantini, R. Coppini, P. Yan, L. M. Loew, M. Campione, L. Bocchi, D. Giulietti, E. Cerbai, C. Poggesi, G. Bub, F. S. Pavone, and L. Sacconi. Real-time optical manipulation of cardiac conduction in intact hearts. The Journal of Physiology, 17:3841-3858, 2018. ISSN 00223751. doi: 10.1113/JP276283. URL http://doi.wiley.com/10.1113/ JP276283, 22, 73, 96

[78] Franziska Schneider, Christiane Grimm, and Peter Hegemann. Biophysics of Channelrhodopsin. Annual Review of Biophysics, 44(1):167-186, 2015. ISSN 1936-122X. doi: 10.1146/annurev-biophys-060414-034014. URL http://www . annualreviews .org/ doi/10.1146/annurev-biophys-060414-034014. 23

[79] Eleanor B Schron, Derek V Exner, Qing Yao, Louise S Jenkins, Jonathan S Steinberg, James R Cook, Steven P Kutalek, Peter L Friedman, Rosemary S Bubien, Richard L 
Page, and Judy Powell. Quality of Life in the Antiarrhythmics Versus Implantable Defibrillators Trial. Circulation, 105(5):589-594, feb 2002. doi: 10.1161/hc0502.103330. URL https : //www . ahajournals .org/doi/10.1161/hc0502.103330, 27

[80] David Shier, Jackie Butler, and Ricki Lewis. Hole's human anatomy \& physiology. McGraw-Hill, 1999. ISBN 0-697-34193-3. 7

[81] Daniel C. Sigg, Paul A. Iaizzo, Yong-Fu Xiao, and Bin He. Cardiac Electrophysiology Methods and Models. Springer US, Boston, MA, 2010. ISBN 978-1-4419-6657-5. doi: 10.1007/978-1-4419-6658-2. 7, 8, 9, 13, 17, 18

[82] Warren Smith and Margaret Hood. Arrhythmias, pages 316-341. ButterworthHeinemann, 2007. ISBN 0-7506-7572-1. doi: 10.1016/B978-075067572-7.50024-2. 13,15

[83] Susan A. Thompson, Craig R. Copeland, Daniel H. Reich, and Leslie Tung. Mechanical coupling between myofibroblasts and cardiomyocytes slows electric conduction in fibrotic cell monolayers. Circulation, 123(19):2083-2093, 2011. ISSN 00097322. doi: 10.1161/CIRCULATIONAHA.110.015057. 54,73

[84] Dhananjay Vaidya, Gregory E. Morley, Faramarz H. Samie, and José Jalife. Reentry and Fibrillation in the Mouse Heart. Circulation Research, 85(2):174-181, 1999. ISSN 0009-7330. doi: 10.1161/01.res.85.2.174. 76

[85] Christoph C. Vogt, Tobias Bruegmann, Daniela Malan, Annika Ottersbach, Wilhelm Roell, Bernd K. Fleischmann, and Philipp Sasse. Systemic gene transfer enables optogenetic pacing of mouse hearts. Cardiovascular Research, 106(2):338-343, 2015. ISSN 17553245. doi: $10.1093 / \mathrm{cvr} / \mathrm{cvv} 004.46,95$

[86] Joseph T. Walsh. Basic Interactions of Light with Tissue, pages 13-26. Springer Netherlands, Dordrecht, 2011. ISBN 978-90-481-8831-4. doi: 10.1007/978-90-481-8831-4_2. URL https://doi .org/10.1007/978-90-481-8831-4_2. 46

[87] Larry Wasserman. Hypothesis Testing and p-values, pages 149-173. Springer New York, New York, NY, 2004. ISBN 978-0-387-21736-9. doi: 10.1007/978-0-387-21736-9_10. URL https ://doi .org/10.1007/978-0-387-21736-9_10. 41

[88] Masaya Watanabe, Iolanda Feola, Rupamanjari Majumder, Wanchana Jangsangthong, Alexander S Teplenin, Dirk L Ypey, Martin J Schalij, Katja Zeppenfeld, and Antoine A F De Vries. Optogenetic manipulation of anatomical re-entry by light-guided generation of a reversible local conduction block. Cardiovascular Research, pages 354-366, 2017. doi: $10.1093 / \mathrm{cvr} / \mathrm{cvx} 003$. 32, 33, 34, 91

[89] Mark S. Wathen, Paul J. DeGroot, Michael O. Sweeney, Alice J. Stark, Mary F. Ot- 
terness, Wayne O. Adkisson, Robert C. Canby, Koroush Khalighi, Christian Machado, Donald S. Rubenstein, and Kent J. Volosin. Prospective randomized multicenter trial of empirical antitachycardia pacing versus shocks for spontaneous rapid ventricular tachycardia in patients with implantable cardioverter-defibrillators: Pacing fast ventricular tachycardia reduces shock therapies. Circulation, 110(17):2591-2596, 2004. ISSN 00097322. doi: 10.1161/01.CIR.0000145610.64014.E4. 27

[90] Matthew Weitzman and Klaus M Hahn. Optogenetic approaches to cell migration and beyond. Current Opinion in Cell Biology, 30:112 - 120, 2014. ISSN 0955-0674. doi: https://doi.org/10.1016/j.ceb.2014.08.004. URL http://www.sciencedirect. com/science/article/pii/S0955067414001021. Cell adhesion and migration. 21

[91] Wilde Arthur A. M. KATP Channel Opening and Arrhythmogenesis, 1994. 39

[92] John C. Williams and Emilia Entcheva. Optogenetic versus electrical stimulation of human cardiomyocytes: Modeling insights. Biophysical Journal, 108(8):1934-1945, 2015. ISSN 15420086. doi: 10.1016/j.bpj.2015.03.032. URL http://dx.doi.org/ $10.1016 / j . b p j .2015 .03 .032 .25$

[93] Echt D S Winkle RA Bach SM Oyer P, Armstrong K, Stinson E B, R A Winkle, E B Stinson, S M Jr Bach, D S Echt, P Oyer, and K Armstrong. Measurement of cardioversion/defibrillation thresholds in man by a truncated exponential waveform and an apical patch-superior vena caval spring electrode configuration. Circulation, 69(4):766, 1984. ISSN 0009-7322. URL http://ovidsp.ovid.com/ovidweb.cgi?T=JS\{\&\}PAGE=reference $\{\&\} \mathrm{D}=$ med2 $\{\&\}$ NEWS $=N\{\&\}$ AN=6697460 $\{\%\} 0$ Ahttp $: / /$ ovidsp.ovid. com/ovidweb. $\operatorname{cgi}$ ? $\mathrm{T}=$ JS $\{\&\}$ PAGE $=$ reference $\{\&\} \mathrm{D}=\operatorname{cctr}\{\&\} \mathrm{NEWS}=\mathrm{N}\{\&\} \mathrm{AN}=\mathrm{CN}-00033710,76$

[94] Lizhi Xu, Sarah R Gutbrod, Andrew P Bonifas, Yewang Su, Matthew S Sulkin, Nanshu Lu, Hyun-Joong Chung, Kyung-In Jang, Zhuangjian Liu, Ming Ying, Chi Lu, R Chad Webb, Jong-Seon Kim, Jacob I Laughner, Huanyu Cheng, Yuhao Liu, Abid Ameen, JaeWoong Jeong, Gwang-Tae Kim, Yonggang Huang, Igor R Efimov, and John a Rogers. 3D multifunctional integumentary membranes for spatiotemporal cardiac measurements and stimulation across the entire epicardium. Nature communications, 5:3329, 2014. ISSN 2041-1723. doi: 10.1038/ncomms4329. URL http://www .nature.com/ncomms/ 2014/140225/ncomms4329/full/ncomms4329.html. 22

[95] Haifeng Ye and Martin Fussenegger. Optogenetic Medicine : Synthetic Therapeutic Solutions Precision-Guided by Light. Cold Spring Harb Perspect Med., pages 1-24, 2019. doi: 10.1101/cshperspect.a034371. 21

[96] Tania Zaglia, Nicola Pianca, Giulia Borile, Francesca Da Broi, Claudia Richter, Marina Campione, Stephan E. Lehnart, Stefan Luther, Domenico Corrado, Lucile Miquerol, and Marco Mongillo. Optogenetic determination of the 
myocardial requirements for extrasystoles by cell type-specific targeting of ChannelRhodopsin-2. Proceedings of the National Academy of Sciences, 112(32): 1509380112-, 2015. ISSN 0027-8424. doi: 10.1073/pnas.1509380112. URL http://www .pnas.org/lookup/doi/10.1073/pnas.1509380112\{\%\}5Cnhttp:// WwW .pnas .org/content/early/2015/07/22/1509380112.abstract.html?etoc. 22, 25, 27, 46, 54, 55, 95

[97] Tania Zaglia, Anna Di Bona, and Marco Mongillo. A Light Wand to Untangle the Myocardial Cell Network. Methods and Protocols, 2(2):34, 2019. doi: 10.3390/ mps2020034. 73

[98] C.M. Zgierski-Johnston, S Ayub, M.C. Fernández, E.A. Rog-Zielinska, F Barz, $\mathrm{O}$ Paul, $\mathrm{P}$ Kohl, and $\mathrm{P}$ Ruther. Cardiac pacing using transmural multi-LED probes in channelrhodopsin-expressing mouse hearts. Progress in Biophysics and Molecular Biology, nov 2019. ISSN 00796107. doi: 10.1016/j.pbiomolbio.2019. 11.004. URL https://doi.org/10.1016/j.pbiomolbio.2019.11.004https:// linkinghub.elsevier.com/retrieve/pii/S0079610719301117, 95, 96

[99] ZooFari. Heart diagram blood flow, 2010. URL https://en.m.wikipedia.org/ wiki/File:Heart_diagram_blood_flow_en.svg. Accessed: 2020-Feb-14. 6 
Affidavit 